TRANSACTIONS OF THE

AMERICAN MATHEMATICAL SOCIETY

Volume 355, Number 4, Pages 1297-1364

S 0002-9947(02)03214-2

Article electronically published on December 5, 2002

\title{
BESOV-MORREY SPACES: FUNCTION SPACE THEORY AND APPLICATIONS TO NON-LINEAR PDE
}

\author{
ANNA L. MAZZUCATO
}

\begin{abstract}
This paper is devoted to the analysis of function spaces modeled on Besov spaces and their applications to non-linear partial differential equations, with emphasis on the incompressible, isotropic Navier-Stokes system and semilinear heat equations. Specifically, we consider the class, introduced by Hideo Kozono and Masao Yamazaki, of Besov spaces based on Morrey spaces, which we call Besov-Morrey or BM spaces. We obtain equivalent representations in terms of the Weierstrass semigroup and wavelets, and various embeddings in classical spaces. We then establish pseudo-differential and para-differential estimates. Our results cover non-regular and exotic symbols. Although the heat semigroup is not strongly continuous on Morrey spaces, we show that its action defines an equivalent norm. In particular, homogeneous BM spaces belong to a larger class constructed by Grzegorz Karch to analyze scaling in parabolic equations. We compare Karch's results with those of Kozono and Yamazaki and generalize them by obtaining short-time existence and uniqueness of solutions for arbitrary data with subcritical regularity. We exploit pseudodifferential calculus to extend the analysis to compact, smooth, boundaryless, Riemannian manifolds. BM spaces are defined by means of partitions of unity and coordinate patches, and intrinsically in terms of functions of the Laplace operator.
\end{abstract}

\section{INTRODUCTION}

The focus of this work is the analysis of a class of function spaces, recently introduced by H. Kozono and M. Yamazaki to obtain critical regularity for the Navier-Stokes equation, and to study its applications to partial differential equations. They are modeled on Besov spaces, but the underlying norm is of Morrey type rather than $L^{p}$ (Definition 2.2). Throughout we will call them Besov-Morrey or BM spaces for short 1

As we will show in the paper, BM spaces share many of the properties of Besov spaces, but they represent local oscillations and singularities of functions more precisely. It follows their interest in the theory of partial differential equations, especially non-linear.

Received by the editors September 24, 2001 and, in revised form, September 9, 2002.

2000 Mathematics Subject Classification. Primary 35S05, 42B35; Secondary 76B03, 42C40, $35 \mathrm{~K} 55$.

Key words and phrases. Navier-Stokes equation, function spaces, Littlewood-Paley, pseudodifferential calculus, global analysis.

This work was completed while the author was on leave from Yale University and visiting the Mathematical Sciences Research Institute (MSRI). Research at MSRI was supported in part by NSF grant DMS-9810361.

${ }^{1}$ An alternative name would be KY spaces, a convention followed in Maz1. 
We start by recalling some notions of fluid dynamics, from which the definition of BM spaces arises quite naturally.

The Navier-Stokes system (NS) on $\mathbb{R}^{n}$ in the incompressible, homogeneous, isotropic case is the following set of equations:

$$
\begin{aligned}
& \partial_{t} u(t, x)-\nu \Delta u(t, x)+u(t, x) \cdot \nabla u(t, x)+\nabla p(t, x)=f(t, x), \\
& \operatorname{div} u(t, x)=0,
\end{aligned}
$$

where the vector field $u(t, x)$ represents the velocity of the fluid at the position $x$ and time $t, \nu$ is the (constant) viscosity coefficient, the scalar field $p(x, t)$ is the pressure, $f(x, t)$ are external body forces. $\nu$ and $f$ are given, while $u$ and $p$ are unknowns.

The system must be complemented with an initial condition $u(0, x)=a(x)$ and some prescribed behavior at infinity to obtain a possibly unique solution.

In many applications, the forcing $f$ admits a potential, which can be included in the pressure term. The pressure appears in the equations only to enforce the incompressibility condition (1.1b). So, it is customary to apply a projection onto the divergence-free vector fields, the so-called Leray projection $\mathbb{P}$, on both sides of (1.1a), and study the (formally) simpler equation

$$
\partial_{t} u(t, x)-\nu \Delta u(t, x)+\mathbb{P} \operatorname{div}(u \otimes u)(t, x)=0 .
$$

The pressure $p$ is then determined by solving a Poisson equation.

Our concern here is with the behavior at fixed viscosity; so $\nu$ is conventionally set equal to one.

It is a classical result (cf. Tay1, Tem]) that short-time, strong, unique solutions to (1.2) exist for sufficiently smooth initial data, e.g., for $a$ in the Sobolev space $H^{k}$, $k>n / 2+1$. On the other hand, in the 1930s, J. Leray $[\overline{\mathrm{Lr}}]$ proved the existence of global weak solutions of finite energy (i.e., $u \in L^{2}$ ), called Leray-Hopf solutions. Uniqueness and full regularity of these solutions is still an open problem. But, if a strong solution exists, then the weak solution coincides with it. Therefore, it is relevant to prove existence and uniqueness under fairly weak assumptions on the initial data.

Mild solutions can be obtained by rewriting (1.2) as the integral equation

$$
u(t, x)=e^{t \Delta} a(x)-\int_{0}^{t} e^{(t-s) \Delta} \mathbb{P} \operatorname{div}(u \otimes u)(s, x) d s,
$$

and then proving that, in suitable function spaces, the right-hand side defines a contraction. Here $a$ does not necessarily have finite energy - its vorticity can be a measure; for instance, see [GM], Ka2, Tay2]. This last situation is important for the vortex ring and vortex sheet problem, where the vorticity $\omega=\operatorname{curl} u$ is respectively a bounded measure supported on a closed curve or surface in $\mathbb{R}^{3}$.

One of the most comprehensive studies in this context is that of M. Cannone, who in his Ph.D. thesis Can (1995) defined the notion of spaces adapted to NS. These are functional Banach spaces $E$ with the additional property

$$
\left\|\psi_{j}(D)(f g)\right\|_{E} \leq \eta_{j}\|f\|_{E}\|g\|_{E}, \quad \forall j \in \mathbb{Z} .
$$

$\psi_{j}(D)$ is the Fourier multiplier with symbol $\psi_{j}(\xi)$, where $\left\{\varphi_{0}(\xi), \psi_{j}(\xi)\right\}$ is a Littlewood-Paley partition of unity subordinate to a dyadic decomposition of $\mathbb{R}^{n}$. The 
numbers $\eta_{j}$ are such that

$$
\sum_{j} 2^{-|j|} \eta_{j}<\infty
$$

The above conditions ensure that the right-hand-side of 1.3 defines a Lipschitz map locally-in-time and, hence, that a short-time, unique solution to NS exists. Examples of adapted spaces include:

(1) $X^{p}\left(\mathbb{R}^{n}\right)=\mathbb{P}\left(L^{p}\left(\mathbb{R}^{n}\right)\right)$ for $p \in(n, \infty)$,

(2) $\mathbb{P}\left(\mathcal{M}_{q}^{p}\left(\mathbb{R}^{n}\right)\right)$ for $p \in(n, \infty)$,

where $\mathcal{M}_{q}^{p}$ is the homogeneous Morrey space (Definition 2.1). Furthermore, every functional Banach space that is also an algebra is adapted, since (1.5) holds with $\eta_{j}$ constant.

In general, (1.4) and (1.5) are not enough to give long-time solutions. However, NS is invariant under an appropriate rescaling of the variables. So, when the norm of the space $E$ also has some scaling behavior, it is quite reasonable to look for global existence by means of time dilations, for example, in the form of self-similar solutions.

In this spirit, G. Karch $\overline{\mathrm{Kr}}$ extended Cannone's work by constructing for each $E$ some auxiliary spaces of distributions:

$$
B E^{\alpha}=\left\{f \in \mathcal{S}^{\prime} \mid\|f\|_{B E^{\alpha}}=\sup _{t>0}\left\{t^{\alpha / 2}\left\|e^{t \Delta} f\right\|_{E}\right\}<\infty\right\}, \quad \alpha \geq 0 .
$$

He was able to prove long-time existence for sufficiently small initial data, if $E$ has scaling degree $-n / q$ and $\alpha=1-n / q$ (so $q \geq n$ ). The solution thus obtained is unique only in a suitable subspace of $B E^{\alpha}$. The advantage of using the space $B E^{\alpha}$ over $E$ is that the norm of the initial data can be small in $B E^{\alpha}$ without being so in $E$. As a matter of fact, if $E=L^{p}$, then $B E^{\alpha}$ is the homogeneous Besov space $B_{p, \infty}^{-\alpha}$, where certain highly oscillatory functions exhibit such behavior [Can]. However, in general, these solutions may not have finite energy.

In 1993, H. Kozono and M. Yamazaki [KY] introduced two new classes of spaces, $\mathcal{N}_{p, q, r}^{s}$ and $N_{p, q, r}^{s}$, which, as mentioned above, will be called homogeneous and inhomogeneous Besov-Morrey spaces.

$N_{p, q, r}^{s}$ is the space of all tempered distributions such that

$$
\|f\|_{N_{p, q, r}^{s}}=\left\|\varphi_{0}(D) f\right\|_{M_{q}^{p}}+\left\{\sum_{j \geq 0}\left(2^{s j}\left\|\psi_{j}(D) f\right\|_{M_{q}^{p}}\right)^{r}\right\}^{1 / r}<\infty,
$$

while $\mathcal{N}_{p, q, r}^{s}$ is the space of all tempered distributions modulo polynomials such that

$$
\begin{aligned}
\|f\|_{\mathcal{N}_{p, q, r}^{s}}= & \left\{\sum_{j \in \mathbb{Z}}\left(2^{s j}\left\|\psi_{j}(D) f\right\|_{\mathcal{M}_{q}^{p}}\right)^{r}\right\}^{1 / r} \\
& =\left\|\left\{2^{s j}\left\|\psi_{j}(D) f\right\|_{\mathcal{M}_{q}^{p}}\right\}_{j=-\infty}^{\infty}\right\|_{\ell^{r}}<\infty,
\end{aligned}
$$

where

$$
\mathcal{M}_{q}^{p} \equiv\left\{f \in L_{\mathrm{loc}}^{q} \mid\|f\|_{\mathcal{M}_{q}^{p}}=\sup _{x_{0} \in \mathbb{R}^{n}} \sup _{0<R} R^{n / p-n / q}\|f\|_{L^{q}\left(B\left(x_{0}, R\right)\right)}<\infty\right\},
$$


and

$$
M_{q}^{p} \equiv\left\{f \in L_{\mathrm{loc}}^{q} \mid\|f\|_{M_{q}^{p}}=\sup _{x_{0} \in \mathbb{R}^{n}} \sup _{0<R \leq 1} R^{n / p-n / q}\|f\|_{L^{q}\left(B\left(x_{0}, R\right)\right)}<\infty\right\},
$$

$B\left(x_{0}, R\right)$ being the closed ball of $\mathbb{R}^{n}$ with center $x_{0}$ and radius $R$.

The indices satisfy $1 \leq q \leq p<\infty, s \in \mathbb{R}, r \in[1, \infty]$. Informally, $s$ is a smoothness index, $p$ is a scaling index, while $r$ can be thought of as an interpolation index. $q$ plays a less important role, but gives "flexibility" and additional properties to BM spaces with respect to Besov spaces. In [KY] Kozono and Yamazaki analyzed NS and a particular class of semi-linear heat equations with initial data in certain BM spaces. They used a fixed-point argument in the spirit of Kato and Weissler's approach for the analysis in $L^{p}$ spaces.

There is considerable interest in studying hydrodynamics on Morrey spaces. As we hinted before, the theory can model the case in which vorticity is a measure supported on singular sets in $\mathbb{R}^{n}$. Moreover, $M_{q}^{p}$ seems to encompass several examples of adapted spaces. Indeed, in three dimensions, under some mild assumptions any adapted space is embedded in $M_{2}^{3}[\mathrm{LMR}]$. At the same time, while BM spaces are strictly larger than Morrey spaces - e.g., p.v. $(1 / x) \in \mathcal{N}_{1,1, \infty}^{0}(\mathbb{R})$, but p.v. $(1 / x) \neq \tilde{M}^{1}(\mathbb{R})$ - they are better behaved under many respects, specifically under the action of pseudo-differential operators, as we discuss extensively in Section 3

In the present work, we strive to combine these different approaches in a unified construction. In particular, we aim at developing the theory of BM spaces, parallel to that of Besov spaces. On one hand, in fact, Morrey spaces are sufficiently close to $L^{p}$ spaces so that essentially the same tools can be used, although there are subtle differences (such as duality and interpolation properties BRV]). On the other hand, we indicated already that Morrey spaces represent the local behavior of a function more precisely (cf. Section 2.2 and the connection with BMO). This quality may play a significant role especially in view of applications to equations other than semi-linear parabolic, where smoothing effects are crucial.

Indeed, Besov spaces have successfully replaced Sobolev spaces in treating the Euler equation [Vi1], [Vi2], and in studying critical well-posedness for wave maps Tat], essentially, because at the critical index, Sobolev spaces do not behave well with respect to point-wise multiplication, while Besov spaces still do for appropriate choices of indices. BM spaces retain this property (cf. Corollary 3.22). In addition, they can be naturally embedded in spaces of higher dimension [KY]. The corresponding result fails for Besov spaces.

Conversely, our analysis is somewhat complicated by the fact that the heat semigroup is not strongly continuous on $\mathcal{N}_{p, q, r}^{s}$ or $N_{p, q, r}^{s}$, fundamentally because smooth functions are not dense. Therefore, it will often be necessary to replace strong convergence with convergence in the weak or weak* topology.

We now give a brief outline of the paper.

In Section 2, we introduce the BM and $B E^{\alpha}$ spaces, and we compare them. Section 2.1 treats $\mathcal{N}_{p, q, r}^{s}$ and $N_{p, q, r}^{s}$, starting with an overview of the theory of Morrey spaces. As expected, most of the features exhibited by BM spaces are analogous to those of Besov spaces. For example, they can be described as real interpolation of powers of the Laplacian acting on $M_{q}^{p}$ and $\mathcal{M}_{q}^{p}$. In this context, we complement the results in $[\mathrm{KY}]$ with some non-interpolation results (Proposition [2.7). 
In view of applications to differential equations, it is important to measure regularity of distributions in $\mathcal{N}_{p, q, r}^{s}$ and $N_{p, q, r}^{s}$. In [KY], Kozono and Yamazaki establish a Sobolev-type embedding for BM spaces:

$$
\begin{aligned}
& N_{p, q, r}^{s} \hookrightarrow C_{*}^{s-n / p}, \\
& \mathcal{N}_{p, q, r}^{s} \hookrightarrow \dot{C}_{*}^{s-n / p},
\end{aligned}
$$

for $s>n / p$, where $C_{*}^{s}$ and $\dot{C}_{*}^{s}$ are respectively inhomogeneous and homogeneous Hölder-Zygmund spaces $\{$ For $s=n / p$, we have

$$
\begin{aligned}
& \mathcal{N}_{p, q, \infty}^{n / p} \subset \dot{C}_{*}^{0}, \\
& \mathcal{N}_{p, q, r}^{n / p} \subset \mathrm{BMO}, r \in[1,2], \\
& \mathcal{N}_{p, q, 1}^{n / p} \subset L^{\infty},
\end{aligned}
$$

BMO being the space of functions of bounded mean oscillation [JN]. The relation with BMO is also addressed in Section 2.2. where we develop a wavelet decomposition for BM spaces in Proposition 2.13] and Proposition [2.14. Following [FJ1], we use a set of smooth wavelets that are not compactly supported, but are "concentrated" on dyadic cubes. In particular, at the critical scaling for NS, Besov-Morrey spaces are not comparable with the space $\mathrm{BMO}^{-1}$ of Koch and Tataru [KoT].

Although wavelets have not led to substantial progress in studying Navier-Stokes, they have proved useful for other (non-parabolic) equations. We are guided here, in particular, by work of M. Vishik [Vi2] on the Euler equation.

Section 2.3 deals with Karch's construction. In Proposition 2.22 we show that $B E^{\alpha}$ can be identified with a homogeneous Besov-type space of negative index, based on $E$, if again the norm of $E$ has some scaling degree. The intuitive reason behind this result is that the symbol of the heat operator $e^{-t|\xi|^{2}}$ behaves like a bump function centered at the origin. By comparison, the norm of $u$ in a Besov space is defined in terms of smooth multipliers $\psi_{j}(D)=\psi\left(2^{-j} D\right)$, where each function $\psi_{j}(\xi)$ is supported on dyadic shells in frequency space. As a matter of fact, for classical Besov spaces it is a well-known result in interpolation theory that an equivalent norm can be obtained in terms of convolutions with the heat kernel Trieb1]. However, our proof is based solely on rescaling arguments and it does not assume that the heat semigroup is strongly continuous on $E$. As a by-product, we obtain an equivalent characterization of $\mathcal{N}_{p, q, \infty}^{s}$ and $N_{p, q, \infty}^{s}$ in terms of thermic functions:

$$
\begin{aligned}
\|f\|_{\mathcal{N}_{p, q, \infty}^{s}} & \approx \sup _{0<t}\left\{t^{-s / 2}\left\|e^{t \Delta} f\right\|_{\mathcal{M}_{q}^{p}}\right\}, \\
\|f\|_{N_{p, q, \infty}^{s}} & \approx \sup _{0<t \leq 1}\left\{t^{-s / 2}\left\|e^{t \Delta} f\right\|_{M_{q}^{p}}\right\} .
\end{aligned}
$$

Consequently, we are able to identify $\mathcal{N}_{p, q, \infty}^{s}$ with $B E^{-s}$, for $s<0$ and $E=\mathcal{M}_{q}^{p}$. This result positively answers a question of G. Karch [Kr].

Section 3 is devoted to the analysis of pseudo-differential operators. Section 3.1 contains a brief introduction to pseudo-differential calculus and results on Fourier multipliers. In $[\mathrm{KY}]$, mapping properties for multipliers of the Mikhlin-Hörmander type were obtained by localizing the symbol to a single dyadic shell.

\footnotetext{
${ }^{2}$ Recall that $C_{*}^{s} \equiv C^{s}$, the usual Hölder space, when $s>0$ is not an integer Trieb3].
} 
Note that, as far as Navier-Stokes on flat Euclidean space is concerned, Fourier multipliers are sufficient. The situation is quite different on manifolds. In Section [3.2, we are then led to consider more general symbols, belonging to the class $S_{1, \delta}^{m}$. Borrowing techniques employed for Sobolev and Hölder spaces [Ma], Ru2], Bour, we establish rather general results; namely, operators with symbols in the class $S_{1, \delta}^{0}$ are bounded on $N_{p, q, r}^{s}$ for all $\delta \in[0,1]$, if $s>0$. This includes the exotic class $S_{1,1}^{0}$, which is relevant in the study of para-differential operators and non-linear equations. For $\delta<1$, good multiplicative properties of the symbols allow us to lift the restriction $s>0$. In the case $q>1$, the same conclusion can be reached directly, using the representation of $N_{p, q, r}^{s}$ as a real interpolation space of powers of $(I-\Delta) M_{q}^{p}$ (Proposition 3.14). Moreover, one is not restricted to smooth symbols, but can work with the class of non-regular symbols $C_{*}^{\ell} S_{1, \delta}^{0}$, as long as $\ell>s>0$, which is the content of Theorem 3.17. Here, too, a stronger result holds if $\delta<1$ by means of symbol smoothing (Corollary 3.20).

In order to treat operators on the homogeneous space $\mathcal{N}_{p, q, r}^{s}$, a different class of symbols must be chosen to take into account possible singularities at the origin. But we do not consider this problem here.

In Section 3.3, we address para-differential calculus. Para-differential operators were introduced by J.-M. Bony [Bo] to study optimal regularity of solutions to non-linear partial differential equations. Here, we use a clever expansion (due to Y. Meyer [Me] ) of a non-linear map $F(u)$, similar to a Taylor polynomial. One is left with a smooth remainder $R(u)$ and a linear part $M_{F}(u, D) u$, where, however, $M_{F}$ is not a differential operator. In fact, if $u \in L^{\infty}, M_{F}$ is a pseudo-differential operator of type $(1,1)$. Since the non-linear term in the Navier-Stokes equation is quadratic, we specialize to $F\left(u_{1}, u_{2}\right)=u_{1} u_{2}$ and prove Moser-type estimates (equation (3.54) ). These combined with the Sobolev embedding (1.11) imply that $N_{p, q, r}^{s}$ is a Banach algebra for $s>n / p$ (Corollary 3.22).

Finally, we compare Meyer's construction to the paraproduct of J.-M. Bony (see again [Bo]), which is obtained by applying symbol smoothing directly to $F$. Various estimates are established here (Corollary 3.23) that again are very similar to those for Sobolev and Hölder spaces.

In Section 4, we address the problem of defining the BM spaces on manifolds. We only deal with the local space $N_{p, q, r}^{s}, q>1$, as pseudo-differential estimates on BM and Morrey spaces [Tay3 are used. The key step is, of course, to replace the multipliers $\psi_{j}(D)$ with appropriate analogs on a curved non-Euclidean geometry. Indeed, operators on manifolds correspond, in general, to equivalence classes of symbols. We look at the simplest case, namely compact, Riemannian manifolds $M$ without boundary, but we plan to consider manifolds with (smooth) boundary in subsequent work, exploiting the analysis developed here. To construct the BM spaces on $M$, we proceeded along two directions.

In Section 4.1] we use a partition of unity and coordinate charts (Definition 4.5), as we show that diffeomorphisms of $\mathbb{R}^{n}$ preserve the topology of $N_{p, q, r}^{s}\left(\mathbb{R}^{n}\right)$. The main ingredient in the proof consists of applying Egorov's Theorem to obtain a complete asymptotic expansion of the symbol of $\chi^{*-1} \circ \psi_{j}(D) \circ \chi^{*}$, for $\chi$ a diffeomorphism of $\mathbb{R}^{n}$ linear outside some compact set, and in studying the behavior of each term in this expansion when $j \rightarrow \infty$. 
In Section 4.2 the topology of $N_{p, q, r}^{s}(M)$ is defined in terms of covariant objects; namely, a family of functions of $\sqrt{-\Delta}$ of the form $\psi_{j}(\sqrt{-\Delta})$, where $\Delta$ is the Laplace operator on $M$.

Again, we first reduce the problem to local coordinates. Then, in Theorem 4.7 we carefully compare the symbols of $\psi_{j}(D)$ and $\psi_{j}(A)$, where $A$ is the operator obtained by transporting the Laplacian on $M$ to $\mathbb{R}^{n}$ via a coordinate chart. An asymptotic formula for the symbol of $\psi_{j}(A)$ can be obtained in terms of the solution operator to the wave equation $\cos (t \sqrt{-\Delta})$, by using geometrical optics methods. We follow Schrader and Taylor [ST], although our approach is rather similar to that of Seeger and Sogge for Besov spaces [SeSo]. However, it is necessary here to bound explicitly the remainder term at each step in the symbol expansion, uniformly in $j$, since no reduction to smooth functions is possible. Finally, $N_{p, q, r}^{s}(M)$ can still be characterized as a real interpolation space of powers of $(I-\Delta)$ acting on $M_{q}^{p}(M)$ (Proposition 4.12).

Section 5 is finally devoted to the study of solutions to certain semi-linear parabolic equations, with an emphasis on Navier-Stokes. Existence and regularity results for this class of equations were obtained in $[\mathrm{KY}]$, when the initial data belong to certain BM spaces of negative index $s$. Similar results can be found in $[\mathrm{Kr}$ ] concerning the spaces $B E^{\alpha}$. Our aim here is to give a unifying approach using the theory developed in the previous sections and extend the analysis to compact manifolds.

Section [5.1] deals with background material and briefly introduces techniques which are used later. As discussed above, we look for mild solutions by applying the contraction mapping theorem or by direct inspection of the Picard iteration. In Section 5.2 we consider scalar perturbations of the heat equation, while section 5.3 treats the Navier-Stokes system.

A typical feature of this approach is the existence of a critical index for the space of initial data - it is $s=n / p-1,1 \leq p \leq \infty$, for NS-which corresponds to so-called limit spaces, characterized by invariance of the norm under the natural scaling of the equation. In the subcritical case, i.e., $s>n / p-1$, one expects (local) existence for all initial data, while an extra condition needs to be imposed in the critical case, but global existence then follows by means of time dilations.

Under the identification (1.13), the results in $[\mathrm{KY}$ are indeed covered by the existence theorem in $\overline{\mathrm{Kr}}$ for the spaces $B E^{\alpha}$, and the condition on the initial data in $[\mathrm{KY}]$ is, in fact, redundant for subcritical indices. Additionally, we show persistence of regularity for spaces with positive index $s$, where condition (1.4) holds. Specifically, $N_{p, q, r}^{s}$ is adapted to NS for

(1) $s>n / 2 p$, if $p>n, 1 \leq q<2, r \in[1, \infty]$,

(2) $s>0$, if $p>n, 2 \leq q \leq p<\infty, r \in[1, \infty]$.

In Section 5.5. we consider the same equations on compact manifolds. In particular, we prove that, provided the Littlewood-Paley operators $\psi_{j}(D)$ are substituted by $\psi_{j}(\sqrt{-\Delta})$, virtually all previous results extend. Because of the finite size of the domain, the behavior for $t \sim 0$ is the relevant question in this case. The analysis is slightly more complicated for NS than for scalar semi-linear equations, since curvature adds an extra term to the equation.

Summarizing, short-time existence and uniqueness for Navier-Stokes holds in $N_{p, q, \infty}^{s}$, if 
- $n / p-1<s<0$ or $s>n / 2 p, p>n$, for arbitrary initial data,

- $s=n / p-1$ for data satisfying an extra condition.

The solution persists for all times for sufficiently small initial data in $\mathcal{N}_{p, q, \infty}^{n / p-1}$.

We conclude by introducing some notation that will be used throughout the paper.

First of all, $\mathcal{F}$ and $\mathcal{F}^{-1}$ denote respectively the Fourier and inverse Fourier transforms, and we set

$$
(\mathcal{F} f)(\xi)=\hat{f}(\xi),\left(\mathcal{F}^{-1} g\right)(x)=\check{g}(x) .
$$

$\mathcal{S}$ is the Schwartz class of rapidly decreasing functions and and its dual $\mathcal{S}^{\prime}$ is the space of tempered distributions. We indicate continuous injections with $\hookrightarrow$.

Unless otherwise noted, we consider functions or distributions on $\mathbb{R}^{n}$, where $n \geq 2$ is fixed, and we usually omit the reference to the underlying space, i.e., we write $L^{p}, \mathcal{M}_{q}^{p}$ for $L^{p}\left(\mathbb{R}^{n}\right), \mathcal{M}_{q}^{p}\left(\mathbb{R}^{n}\right)$, and so on.

With $e^{t \Delta}, t \geq 0$, we mean the Weierstrass or heat semigroup and $p_{t}$ is the corresponding Schwartz kernel. Recall that

$$
p_{t}(x)=\frac{1}{(4 \pi t)^{n / 2}} \exp \left\{\frac{-|x-y|^{2}}{4 t}\right\} .
$$

$D$ stands for the differential operator $(1 / i) \nabla$, where $i=\sqrt{-1}$, so that $\mathcal{F}\left(D_{j} f\right)(\xi)$ $=\xi_{j} \hat{f}$. If $P$ is a pseudo-differential operator, $\sigma(P)$ will denote its symbol and $\sigma_{0}(P)$ its principal symbol.

Finally, we denote any irrelevant constant with $C$, unless we want to emphasize that $C$ depends on certain indices, say $p$ or $q$, in which case we write $C_{p, q}$ or $C(p, q)$.

\section{Two Classes of Function Spaces}

It is a common experience that different time and length scales appear in the motion of fluids and that certain phenomena seem to be independent of the choice of such scales. Mathematically, scaling properties of non-linear differential equations are an important tool in analyzing solutions, especially when the non-linearity is a homogeneous function.

Invariance under some scaling law allows us, for example, to construct new solutions from known ones. Also, self-similar solutions, if they exist, persist for all times. For the Navier-Stokes system, global-in-time existence of smooth solutions has not been proven to this day, except for special cases.

It is, therefore, natural to choose initial data in function spaces that are constructed by imposing some scaling behavior. This behavior can be tailored to the type of non-linearity in the equation.

2.1. Besov-Morrey spaces. Due to the smoothing effect of the heat semigroup, solutions to the Navier-Stokes equation exist when the initial data have minimal regularity - for example, they can be distributions whose associated vorticity is a singular measure (see [GM], GMO]). This motivates in part the study of the Navier-Stokes and other evolution equations in Morrey spaces and generalizations. Examples of such spaces were constructed by H. Kozono and M. Yamazaki ([KY]).

We start by recalling the definition and some of the properties of the Morrey spaces $\mathcal{M}_{q}^{p}$ and $M_{q}^{p}$. 
Definition 2.1. For $p$ and $q$ satisfying $1 \leq q \leq p<\infty$, define the homogeneous Morrey space, $\mathcal{M}_{q}^{p}$, and the inhomogeneous or local Morrey space, $M_{q}^{p}$, respectively as

$$
\mathcal{M}_{q}^{p} \equiv\left\{f \in L_{l o c}^{q} \mid\|f\|_{\mathcal{M}_{q}^{p}}=\sup _{x_{0} \in \mathbb{R}^{n}} \sup _{0<R} R^{n / p-n / q}\|f\|_{L^{q}\left(B\left(x_{0}, R\right)\right)}<\infty\right\}
$$

and

$$
M_{q}^{p} \equiv\left\{f \in L_{l o c}^{q} \mid\|f\|_{M_{q}^{p}}=\sup _{x_{0} \in \mathbb{R}^{n}} \sup _{0<R \leq 1} R^{n / p-n / q}\|f\|_{L^{q}\left(B\left(x_{0}, R\right)\right)}<\infty\right\},
$$

where $B\left(x_{0}, R\right)$ is the closed ball of $\mathbb{R}^{n}$ with center $x_{0}$ and radius $R$.

It is clear that $\mathcal{M}_{q}^{p} \subset M_{q}^{p}$ and that they agree on compactly supported elements. Moreover, by Hölder's inequality,

$$
M_{p}^{p} \subset M_{q}^{p} \subset M_{1}^{p} .
$$

Other embeddings that will be useful later are

$$
\begin{array}{ll}
M_{q}^{p} \subset M_{q}^{p^{\prime}} & \text { if } 1 \leq q \leq p^{\prime} \leq p<\infty, \\
M_{r}^{p} \subset M_{q}^{p} \text { and } \mathcal{M}_{r}^{p} \subset \mathcal{M}_{q}^{p} & \text { if } 1 \leq q<r \leq p<\infty, \\
M_{r}^{p^{\prime}} \subset M_{q}^{p} & \text { if } 1 \leq q \leq \min \{r, p\} \leq \max \{r, p\} \leq p^{\prime}<\infty .
\end{array}
$$

For example, let $n=1$ and pick $f(x)=1 / \sqrt{x}$. Then, $f \in M_{q}^{p}$ for $1 \leq q<p \leq 2$, but $f \notin M_{q}^{p}$ for $p>2$ or $q=p=2$. On the other hand, $L^{\infty} \subset M_{q}^{p}$ for all values of $p$ and $q$.

Morrey spaces can be seen as a complement to $L^{p}$ spaces. As a matter of fact, $\mathcal{M}_{p}^{p} \equiv L^{p}$ and $\mathcal{M}_{q}^{p} \subset L^{q}$. They are part of a larger class, that of Morrey-Campanato spaces $\mathcal{L}_{q, \lambda}$, which also include Lipschitz spaces and BMO. $M_{q}^{p}$ corresponds to the choice of parameter $\lambda=n(1-q / p)$ (see $[\mathrm{Pe}]$ ).

Morrey spaces describe local regularity more precisely than $L^{p}$. For example, Morrey's Lemma gives

$$
M_{1}^{p} \subset C_{*}^{-n / p}\left(\mathbb{R}^{n}\right) .
$$

Note that it is stronger than the usual Sobolev embedding, since $M_{1}^{p}$ is strictly larger than $L^{p}$. (2.5) is a consequence of the characterization below in terms of heat kernel estimates Tay3].

If $f \in L_{\mathrm{loc}}^{1}$, then

$$
f \in M_{1}^{p} \longleftrightarrow p_{r} *|f| \leq C r^{-n / 2 p}
$$

for $0<r \leq 1$ and

$$
f \in \mathcal{M}_{1}^{p} \longleftrightarrow p_{r} *|f| \leq C r^{-n / 2 p},
$$

for $0<r$, with a similar estimate for $q>1$. These are sharp results. Consequently, the heat semigroup is not strongly continuous on Morrey spaces.

As anticipated in the Introduction, some of the interest for Morrey spaces in fluid mechanics is related to spaces of measures. Most of the properties carry over if we replace the integral of $f$ in Definition 2.1] with the total variation of a Radon measure $\mu$. The corresponding space is sometimes indicated by $\tilde{\mathcal{M}}^{p}$ Tay3 and $\mathcal{M}_{1}^{p}$ corresponds to the subspace of measures absolutely continuous with respect to the Lebesgue measure.

Following $[\mathrm{KY}]$, we now define Besov spaces based on $M_{q}^{p}$ and $\mathcal{M}_{q}^{p}$. 
We first recall the concept of Littlewood-Paley partition of unity, $\left\{\psi_{j}\right\}, j \in$ $\mathbb{Z}$. The functions $\psi_{j}(\xi)$ are constructed as follows. Let $\varphi_{0}$ be a smooth function supported in the ball $B(0,1)$, such that $\varphi_{0} \equiv 1$ for $0 \leq|\xi| \leq 2 / 3$. Set $\varphi_{j}(\xi)=$ $\varphi_{0}\left(\xi / 2^{j}\right)$, so that $\varphi_{j}$ is supported on the ball $B\left(0,2^{j}\right)$. Define $\psi_{j}=\varphi_{j+1}-\varphi_{j}$. Note that $\psi_{j}(\xi)=\psi_{0}\left(\xi / 2^{j}\right) . \psi_{j}$ is supported on the dyadic shell $D_{j}=\left\{\xi \in \mathbb{R}^{n} \mid 2^{j-1}<\right.$ $\left.|\xi|<2^{j+1}\right\}$. The collection of all shells $D_{j}$, as $j$ runs over the integers, covers $\mathbb{R}_{\xi}^{n} \backslash\{0\}$, with $D_{\ell} \cap D_{k}=\emptyset$ only if $|k-\ell|>1$. Although the width of each shell doubles in size as $j$ increases, $D_{j}$ is still centered around the frequency $|\xi|=2^{j}$. In fact, the $\psi_{j}$ 's act as band filters in the language of digital processing, while the $\phi_{j}$ are low pass filters.

If $f \in \mathcal{S}^{\prime}$, then

$$
f=\left(\varphi_{0}+\sum_{j \geq 0} \psi_{j}\right) f
$$

with convergence in the sense of distributions (telescopic sum). On the other hand, in general, $\left(\sum_{j \in \mathbb{Z}} \psi_{j}\right) f \neq f$, since $\psi_{j} u=0$ in $\mathcal{S}^{\prime}$ for every distribution $u$ that is supported at the origin.

Finally, let $\psi_{j}(D)$ be the Fourier multiplier with symbol $\psi_{j}$ defined as

$$
\psi(D) u(x)=\mathcal{F}^{-1}\left(\psi_{j} \cdot \hat{u}\right)(x)=\int_{\mathbb{R}^{n}} \psi_{j}(\xi) \hat{u}(\xi) e^{i x \cdot \xi} d \xi, \quad u \in \mathcal{S} .
$$

Definition 2.2. For $1 \leq q \leq p<\infty, r \in[1, \infty]$, and $s \in \mathbb{R}$, we say that $f \in \mathcal{S}^{\prime}$ belongs to the space $N_{p, q, r}^{s}$ if

$$
\|f\|_{N_{p, q, r}^{s}}=\left\|\varphi_{0} f\right\|_{M_{q}^{p}}+\left\{\sum_{j \geq 0}\left(2^{s j}\left\|\psi_{j}(D) f\right\|_{M_{q}^{p}}\right)^{r}\right\}^{1 / r}<\infty,
$$

and, similarly, we say that the equivalence class of distributions modulo polynomials, $f \in \mathcal{S}^{\prime} / \mathcal{P}$, belongs to the space $\mathcal{N}_{p, q, r}^{s}$ if

$$
\begin{aligned}
\|f\|_{\mathcal{N}_{p, q, r}^{s}}= & \left\{\sum_{j \in \mathbb{Z}}\left(2^{s j}\left\|\psi_{j}(D) f\right\|_{\mathcal{M}_{q}^{p}}\right)^{r}\right\}^{1 / r} \\
& =\left\|\left\{2^{s j}\left\|\psi_{j}(D) f\right\|_{\mathcal{M}_{q}^{p}}\right\}_{j=-\infty}^{\infty}\right\|_{\ell^{r}}<\infty .
\end{aligned}
$$

The spaces $\mathcal{N}_{p, q, r}^{s}$ and $N_{p, q, r}^{s}$ are hence defined respectively as the homogeneous and inhomogeneous Besov space $\dot{B}_{p, r}^{s}, B_{p, r}^{s}$ with $\mathcal{M}_{q}^{p}$ and $M_{q}^{p}$ replacing $L^{p}$. For brevity, we will call $\mathcal{N}_{p, q, r}^{s}$ and $N_{p, q, r}^{s}$ respectively the homogeneous BM space and inhomogeneous BM space.

It is not difficult to see that different choices for the family of functions $\left\{\psi_{j}\right\}$ give equivalent norms, as long as the $\psi_{j}$ form a Littlewood-Paley partition of unity.

Remark 2.3. One could consider constructing a non-local version of $N_{p, q, r}^{s}$ by taking distributions such that $\left\|\left\{2^{j s}\left\|\psi_{j}(D) f\right\|_{M_{q}^{p}}\right\}_{j \in \mathbb{Z}}\right\|_{\ell^{r}}<\infty$. However, $M_{q}^{p}$ is not invariant under dilations, and so the norm of the corresponding homogeneous space would not have a definite scaling degree.

\footnotetext{
${ }^{3}$ See also Section 3.1
} 
As discussed in [KY], the spaces $\mathcal{N}_{p, q, \infty}^{s}$ and $N_{p, q, \infty}^{s}$ are strictly larger then Morrey spaces; for example, the principal value distribution p.v. $\frac{1}{x}$ belongs to $\mathcal{N}_{1,1, \infty}$, but not to $\mathcal{M}_{1}^{1}$. On the other hand, their behavior in frequency space is simpler and they are a better candidate for the analysis of partial differential equations, as we aim to show in the paper. They are also strictly larger than the corresponding Besov spaces, when $p>q$.

Let us, again, collect some useful inclusion relations. From (2.4) and the fact that $\ell^{r} \subset \ell^{r^{\prime}}$ if $r \leq r^{\prime}$, it follows immediately that

$$
N_{p, q, r}^{s} \subset N_{p, q^{\prime}, r^{\prime}}^{s} \text { and } \mathcal{N}_{p, q, r}^{s} \subset \mathcal{N}_{p, q^{\prime}, r^{\prime}}^{s}
$$

if $1 \leq q^{\prime} \leq q \leq p<\infty$ and $1 \leq r \leq r^{\prime} \leq \infty$. Hölder's inequality for $\ell^{r}$-spaces gives instead

$$
N_{p, q, r}^{s} \subset N_{p, q, r^{\prime}}^{s^{\prime}}
$$

for every $r, r^{\prime} \in[1, \infty]$ if $s \geq s^{\prime}$. Hence, the inhomogeneous spaces form a scale. In particular, $s$ can be interpreted as a "smoothness" index, i.e., elements of $N_{p, q, r}^{s}$ are more and more regular as $s$ increases (cf. Theorem 2.4 below). However, we never have $\mathcal{N}_{p, q, r}^{s} \subset \mathcal{N}_{p, q, r^{\prime}}^{s^{\prime}}$, intuitively because low and high frequencies "weigh the same" in the Fourier representation of $f \in \mathcal{N}_{p, q, r}^{s}$.

In general, a Littlewood-Paley component $\psi_{j}(D) f$ may not decay at infinity, but it is slowly increasing by virtue of the Paley-Wiener theorem. The Morrey norm essentially controls the growth over balls of given radius. Therefore, by carefully decomposing the convolution $\psi_{j}(D) f=\breve{\psi}_{j} * f$, one can prove the following Sobolevtype embedding theorem.

Theorem 2.4 ( $[\mathrm{KY}])$. The following inclusions hold:

$$
N_{p, q, r}^{s} \subset B_{\infty, r}^{s-n / p}, \quad \mathcal{N}_{p, q, r}^{s} \subset \dot{B}_{\infty, r}^{s-n / p},
$$

if $1 \leq q \leq p<\infty, s \in \mathbb{R}$ and $r \in[1, \infty]$.

In the critical case $s=n / p$; in particular, we have (we state only the homogeneous case for simplicity)

$$
\begin{aligned}
\mathcal{N}_{p, q, \infty}^{n / p} & \subset \dot{B}_{\infty, \infty}^{0} \equiv \dot{C}_{*}^{0}, \\
\mathcal{N}_{p, q, r}^{n / p} & \subset \dot{B}_{\infty, 2}^{0} \subset \mathrm{BMO}, r \in[1,2], \\
\mathcal{N}_{p, q, 1}^{n / p} & \subset L^{\infty},
\end{aligned}
$$

where $\dot{C}_{*}^{s}$ are homogeneous Zygmund spaces Trieb2 and BMO is the space of functions of bounded mean oscillations, introduced by John and Nirenberg [JN]. Recall that $C_{*}^{s} \equiv C^{s}$ if $s>0$ is not an integer, but $C^{k} \subset C_{*}^{k}$ for $k \in \mathbb{Z}_{+}$.

Potential estimates give $(-\Delta)^{-n / 2 p} \mathcal{M}_{q}^{p} \hookrightarrow$ BMO Miy. So, (2.15) follows immediately from a theorem of Littlewood-Paley type for Morrey spaces [Maz2].

From Theorem 2.4. completeness of BM spaces follows (cf. proof of Lemma 2.21). Also, the inclusions $\mathcal{N}_{p, q, r}^{s} \hookrightarrow \mathcal{S}^{\prime} / \mathcal{P}, N_{p, q, r}^{s} \hookrightarrow \mathcal{S}^{\prime}$ are continuous

In general, elements of $\mathcal{N}_{p, q, r}^{s}$ belong only to $\mathcal{S}^{\prime} / \mathcal{P}$, since the kernel of each operator $\psi_{j}(D)$ contains those distributions for which the Fourier Transform is supported at the origin. However, for certain values of $p, q$ and $s$, one can canonically identify each class $f$ in $\mathcal{N}_{p, q, r}^{s}$ with a distribution. To do so, note that for each fixed $l \in \mathbb{Z}$,

\footnotetext{
${ }^{4} \mathcal{S}^{\prime} / \mathcal{P}$ is endowed with the quotient topology.
} 
$\sum_{j=l}^{\infty} \psi_{j}(D) f$ converges to an element, $f_{(l)}$, of $\mathcal{S}^{\prime}$. Using the fact that the supports of $\psi_{j}(\xi)$ and $\psi_{k}(\xi)$ do not overlap for $|j-k|>2$, one can show that actually the sequence $\left\{f_{(l)}\right\}$ converges in $\mathcal{N}_{p, q, r}^{s}$ and to $f$. So, it is enough to prove that $\left\{f_{(l)}\right\}$ converges in $\mathcal{S}^{\prime}$. This will depend on the values of $p, q$ and $s$.

Proposition 2.5 ([KY] $)$. Let $s<n / p$, or $s=n / p$ and $r=1$. Then, the sequence $\left\{f_{(l)}\right\}$ converges in $\mathcal{S}^{\prime}$ and the limit is the canonical representative of the class $f$.

The spaces $\mathcal{N}_{p, q, r}^{s}$ and $N_{p, q, r}^{s}$ can also be described as real interpolation spaces, in the same fashion as the classical Besov spaces are.

Proposition $2.6([\mathrm{KY}])$. For $s_{1} \neq s_{2} \in \mathbb{R}$ and $\theta \in(0,1)$, the following interpolation relations hold:

(a) $\quad\left(N_{p, q, r_{1}}^{s_{1}}, N_{p, q, r_{2}}^{s_{2}}\right)_{\theta, r}=N_{p, q, r}^{s} \quad$ and $\left(\mathcal{N}_{p, q, r_{1}}^{s_{1}}, \mathcal{N}_{p, q, r_{2}}^{s_{2}}\right)_{\theta, r}=\mathcal{N}_{p, q, r}^{s}$

(b) $\quad\left((I-\Delta)^{-s_{1} / 2} M_{q}^{p},(I-\Delta)^{-s_{2} / 2} M_{q}^{p}\right)_{\theta, r}=N_{p, q, r}^{s} \quad$ and

$$
\left((-\Delta)^{-s_{1} / 2} \mathcal{M}_{q}^{p},(-\Delta)^{-s_{2} / 2} \mathcal{M}_{q}^{p}\right)_{\theta, r}=\mathcal{N}_{p, q, r}^{s}
$$

where $s=(1-\theta) s_{1}+\theta s_{2}, r, r_{1}, r_{2} \in[1, \infty]$, and $1 \leq q \leq p<\infty$.

The proof is essentially an adaptation of the $L^{p}$ case. Other useful relations follow in a similar fashion. For the sake of brevity, we consider homogeneous spaces only.

Proposition 2.7. Again, let $s, s_{j} \in \mathbb{R}, 1 \leq q, q_{j} \leq p \leq \infty, r, r_{j} \in[1, \infty], j=1,2$, $\theta \in(0,1)$. Then

(1) $\left(\mathcal{N}_{p, q, r_{1}}^{s}, \mathcal{N}_{p, q, r_{2}}^{s}\right)_{\theta, r^{*}}=\mathcal{N}_{p, q, r^{*}}^{s}$, if $\frac{1}{r^{*}}=\frac{1-\theta}{r_{1}}+\frac{\theta}{r_{2}}$,

(2) $\left(\mathcal{N}_{p, q_{1}, r_{1}}^{s_{1}}, \mathcal{N}_{p, q_{2}, r_{2}}^{s_{2}}\right)_{\theta, q^{*}} \subset \mathcal{N}_{p, q^{*}, r^{*}}^{s}$, if $s=(1-\theta) s_{1}+\theta s_{2}, \frac{1}{r^{*}}=\frac{1-\theta}{r_{1}}+\frac{\theta}{r_{2}}$, $\frac{1}{q^{*}}=\frac{1-\theta}{q_{1}}+\frac{\theta}{q_{2}}$ and $q^{*}=r^{*}$.

Proof. Following [BL] (pp. 149-153), it is enough to show that $\mathcal{N}_{p, q, r}^{s}$ is a retract of $\dot{\ell}_{r}^{s}\left(\mathcal{M}_{q}^{p}\right)$, where

$$
\dot{\ell}_{r}^{s}\left(\mathcal{M}_{q}^{p}\right)=\left\{\left\{\alpha_{n}\right\}, \alpha_{n} \in \mathcal{M}_{q}^{p}, n \in \mathbb{Z} \mid\left(\sum_{j=-\infty}^{+\infty} 2^{j s r}\left\|\alpha_{j}\right\|_{\mathcal{M}_{q}^{p}}^{r}\right)^{1 / r} \leq \infty\right\} .
$$

For $\alpha=\left\{\alpha_{j}\right\} \in \dot{\ell}_{r}^{s}\left(\mathcal{M}_{q}^{p}\right)$, define a map $\mathcal{I}$ by

$$
\mathcal{I}(\alpha)=\sum_{j} \Psi_{j} * \alpha_{j}
$$

where $\Psi_{j}=\sum_{k=j-1}^{j+1} \psi_{k}$; so, $\Psi_{j}$ is identically one on the support of $\psi_{j}$. Similarly, for $f \in \mathcal{S}^{\prime}$, define a map $\mathcal{J}$ by

$$
\mathcal{J}(f)_{j}=\psi_{j} * f .
$$


Then, clearly $\mathcal{I} \circ \mathcal{J}$ is the identity map on $\mathcal{S}^{\prime} / \mathcal{P}$. To prove our claim, we need to show that

and

$$
\mathcal{J}: \mathcal{N}_{p, q, r}^{s} \rightarrow \dot{\ell}_{r}^{s}\left(\mathcal{M}_{q}^{p}\right)
$$

$$
\mathcal{P}: \dot{\ell}_{r}^{s}\left(\mathcal{M}_{q}^{p}\right) \rightarrow \mathcal{N}_{p, q, r}^{s}
$$

boundedly. But $\|\mathcal{J}(f)\|_{\dot{\ell}_{r}^{s}\left(\mathcal{M}_{q}^{p}\right)} \approx\|f\|_{\mathcal{N}_{p, q, r}^{s}}$ by definition. Vice versa, since $\Psi_{j} * \psi_{k} \equiv$ 0 if $|k-j|>1$,

$$
\begin{aligned}
\|\mathcal{P}(\alpha)\|_{\mathcal{N}_{p, q, r}^{s}} & \leq C\left[\sum_{k \in \mathbb{Z}}\left(2^{k s}\left\|\psi_{k} * \alpha_{k}\right\|_{\left.\mathcal{M}_{q}^{p}\right)^{r}}\right]^{1 / r}\right. \\
& \leq C\|\alpha\|_{\dot{\ell}_{r}^{s}\left(\mathcal{M}_{q}^{p}\right)} .
\end{aligned}
$$

The last inequality follows from the following simple observation, which will be frequently exploited.

Remark 2.8. If $E$ is any normed vector space of distributions, whose norm is translation-invariant, then the Fourier multiplier $\phi(D)$ is bounded on $E$ for all $\phi$ such that $\check{\phi} \in L^{1}$ and

$$
\|\phi(D)\|_{\text {op }} \leq\|\check{\phi}\|_{L^{1}} .
$$

In particular, the Littlewood-Paley operators are uniformly bounded with

$$
\left\|\psi_{j}(D)\right\|_{\text {op }} \leq 1, \forall j .
$$

To conclude the proof, we use the following results on interpolation of $\dot{\ell}_{r}^{s}(A)$ spaces, where $A$ is any Banach space ([BL, pp. 121-124):

$$
\left(\dot{\ell}_{r_{1}}^{s}(A), \dot{\ell}_{r_{2}}^{s}(A)\right)_{\theta, r^{*}}=\dot{\ell}_{r^{*}}^{s}(A),
$$

which proves part (1), and

$$
\left(\dot{\ell}_{r_{1}}^{s_{1}}\left(A_{1}\right), \dot{\ell}_{r_{2}}^{s_{2}}\left(A_{2}\right)\right)_{\theta, r^{*}}=\dot{\ell}_{r^{*}}^{s}\left(\left(A_{1}, A_{2}\right)_{\theta, q^{*}}\right)
$$

if $r^{*}=q^{*}$. Part 2 now follows from the inclusion $\left(\mathcal{M}_{q_{1}}^{p}, \mathcal{M}_{q_{2}}^{p}\right)_{\theta, q^{*}} \subset \mathcal{M}_{q^{*}}^{p}$, which is in turn a simple consequence of interpolation of $L^{q}$ spaces.

Contrary to the case of Besov spaces, the inclusion in part 2 is strict. The opposite inclusion does not hold, because Morrey spaces are not interpolation spaces. As shown in [BRV], it is possible to construct operators $T$ bounded from $\mathcal{M}_{q_{j}}^{p}$ into $L^{q_{j}}$ that are not bounded from $\mathcal{M}_{q_{*}}^{p}$ into $L^{q^{*}}$.

The above proposition will be useful in Section 3, when proving regularity of pseudo-differential operators in the spaces $\mathcal{N}_{p, q, r}^{s}$ and $N_{p, q, r}^{s}$.

2.2. Wavelet decomposition. In this section, we are going to develop a decomposition of elements of $\mathcal{N}_{p, q, r}^{s}$ and $N_{p, q, r}^{s}$ in terms of quasi-orthogonal "smooth wavelets", similar to that obtained by Frazier and Jawerth for Besov spaces [FJ1]. The type of wavelets used here are not compactly supported, although they are concentrated on compact sets (dyadic cubes); on the other hand, they have infinitely many vanishing moments.

Wavelets have been used in the analysis of the Navier-Stokes equation [Fe, yet there does not seem to be a clear advantage over the Littlewood-Paley approach 
(cf. [Can]). On the other hand, wavelets have been successful in treating the Euler equation, where there is no smoothing effect of the heat kernel [Vi2].

Let us begin defining dyadic cubes $Q_{\nu k}$, for $\nu \in \mathbb{Z}$ and $k \in \mathbb{Z}^{n}$ :

$$
Q_{\nu k}=\left\{x \in \mathbb{R}^{n} \mid 2^{-\nu} k_{i} \leq x_{i} \leq 2^{-\nu}\left(k_{i}+1\right), i=1, \ldots, n\right\} .
$$

For fixed $\nu$, the collection $\left\{Q_{\nu k}, k \in \mathbb{Z}^{n}\right\}$ tiles the whole space and the cubes are pairwise disjoint. Each cube $Q=Q_{\nu k}$ is uniquely identified by its length $\ell(Q)=2^{-\nu}$ and a preferred corner $x_{Q}=2^{-\nu} k$. We also denote the volume of the cube $Q$ with $|Q|$.

The smooth wavelets we are going to employ arise naturally from LittlewoodPaley components. The first step is contained in the following lemma (see [FJ1]).

Lemma 2.9. Let $f \in \mathcal{S}^{\prime} / \mathcal{P}$ and let $\left\{\psi_{\nu}\right\}$ be a (homogeneous) Littlewood-Paley partition of unity. Then

$$
f(\cdot)=\sum_{\nu \in \mathbb{Z}} 2^{-n \nu} \sum_{k \in \mathbb{Z}^{n}} \check{\psi}_{\nu} * f\left(2^{-\nu} k\right) \check{\psi}_{\nu}\left(\cdot-2^{-\nu} k\right),
$$

where the convergence is in $\mathcal{S}^{\prime} / \mathcal{P}$ and, as before, $\psi_{\nu}(\xi)=\psi\left(\xi / 2^{\nu}\right)$.

The main idea behind the proof is to exploit the compact support of $\psi_{\nu}$ to extend $\psi_{\nu} \hat{f}$ to a periodic function of period $2^{\nu+1} \pi$ in $\mathbb{R}_{\xi}^{n}$, and then use Fourier series to represent it as a discrete sum of "wavenumbers" $2^{-\nu} k$ coupled to the frequency $\xi$. $f$ is now a superposition of elements of a given family of test functions with coefficients equal to the value of $f_{\nu}=\check{\psi}_{\nu} * f$ at the sampling points $2^{-\nu} k$.

For notational convenience, as we will be working in physical space $\mathbb{R}_{x}^{n}$, we set

$$
\check{\psi}_{\nu}(x)=\sigma_{\nu}(x)=2^{\nu n} \sigma\left(2^{\nu} x\right), \quad \sigma=\check{\psi}_{0},
$$

and

$$
\sigma_{\nu k}(x)=\sigma\left(2^{\nu} x-k\right) .
$$

Then, after some simple manipulations, (2.24) becomes

$$
f=\sum_{\nu} 2^{-\nu n} \sum_{k}\left(\sigma_{\nu k}, f\right) \sigma_{\nu k}
$$

which is an almost orthogonal decomposition, since $\left(\sigma_{\nu k}, \sigma_{\mu l}\right)=0$ if $|\nu-\mu|>1$, or anyway negligible if $|k-l|$ is large enough. Each $\sigma_{\nu k}$ is a smooth wavelet concentrated on the dyadic cube $Q_{\nu k}$ with infinite vanishing moments, i.e.,

$$
\begin{gathered}
\int_{\mathbb{R}^{n}} x^{M} \sigma_{\nu k}(x) d x=0, \\
\left|\sigma_{\nu k}(x)\right| \leq C_{M}\left(1+2^{\nu}\left(x-2^{-\nu} k\right)\right)^{-M}
\end{gathered}
$$

for all $M \geq 0$.

The next step is to rewrite the norm of each Littlewood-Paley component $f_{\nu}$ appropriately in terms of dyadic cubes. For $L^{p}$ spaces, a classical result of PlancherelPolya holds [FJ1].

Lemma 2.10. Let $0<p \leq \infty, \nu \in \mathbb{Z}, f \in \mathcal{S}^{\prime}$. Assume Supp $\hat{f} \subset B\left(0,2^{\nu}\right)$. Then,

$$
\left(\sum_{k \in \mathbb{Z}^{n}} \sup _{Q_{\nu k}}|f(x)|^{p}\right)^{1 / p} \leq C_{n, p} 2^{\nu(n / p)}\|f\|_{L^{p}} .
$$


We will prove a similar lemma in the case of Morrey spaces. Clearly, we do not expect to have a full sum over the wave-vectors $k$, but only a partial sum.

Lemma 2.11. Let $1 \leq q \leq p<\infty, \nu \in \mathbb{Z}, f \in \mathcal{S}^{\prime}$. Suppose Supp $\hat{f} \subset B\left(0,2^{\nu}\right)$. Then,

$$
\begin{gathered}
\left(\sup _{\ell(J) \geq 2^{-\nu}}\left(\frac{1}{|J|}\right)^{1-q / p} \sum_{Q_{\nu k} \subset J}|Q|^{1-q / p} \sup _{Q_{\nu k}}|f(x)|^{q}\right)^{1 / q} \\
\leq C_{n, p, q} 2^{\nu(n / p)}\|f\|_{\mathcal{M}_{q}^{p}},
\end{gathered}
$$

where $J$ are dyadic cubes.

Proof. We can choose test functions $\sigma_{\nu}$ satisfying all the hypotheses of Lemma 2.9 such that $\hat{\sigma}_{\nu} \equiv 1$ on the support of $\hat{f}$. Hence, from (2.24),

$$
\begin{aligned}
f(x+y)=\sigma_{\nu} * f_{y}(x) & =2^{-\nu n} \sum_{k \in \mathbb{Z}^{n}} f\left(2^{-\nu} k+y\right) \sigma_{\nu}\left(x-2^{-\nu} k\right) \\
& =\sum_{k \in \mathbb{Z}^{n}} f\left(2^{-\nu} k+y\right) \sigma_{\nu k}(x),
\end{aligned}
$$

with $f_{y}(x)=f(x+y)$. But $\sigma_{\nu k}$ is concentrated on the dyadic cube $Q_{\nu k}$. Therefore (2.26b) easily gives with $M=n+1$,

$$
\begin{aligned}
\sup _{z \in Q_{\nu k}}|f(z)| & \leq \sup _{x \in Q_{\nu 0}}|f(x+y)| \\
& \leq \sup _{|x|<2^{-\nu} \sqrt{n}}|f(x+y)| \\
& \leq C_{n} \sum_{l \in \mathbb{Z}^{n}} f\left(2^{-\nu} l+y\right) \sup _{|x|<2^{-\nu} \sqrt{n}}\left|\sigma_{\nu l}(x)\right| \\
& \leq C_{n} \sum_{l \in \mathbb{Z}^{n}} f\left(2^{-\nu} l+y\right)(1+|l|)^{-(n+1)},
\end{aligned}
$$

where $y$ is some fixed point in $Q_{\nu k}$. Using the triangle inequality $(q=1)$ or Hölder's inequality $(q>1)$, and integrating both sides with respect to $y$ gives

$$
\begin{aligned}
2^{-\nu n} \sup _{z \in Q_{\nu k}}|f(z)|^{q} & \leq C_{n, q} \sum_{l \in \mathbb{Z}^{n}}(1+|l|)^{-(n+1)} \int_{Q_{\nu k}}\left|f\left(2^{-\nu} l+y\right)\right|^{q} d y \\
& \leq C_{n, q} \sum_{l \in \mathbb{Z}^{n}}(1+|l|)^{-(n+1)} \int_{Q_{\nu k+l}}|f(y)|^{q} d y .
\end{aligned}
$$

Clearly, a dyadic cube $J$ can be covered by cubes of length $2^{-\nu}$ only if $\ell(J) \geq 2^{-\nu}$, so that

$$
\begin{aligned}
\sup _{\ell(J) \geq 2^{-\nu}} \sum_{Q_{\nu k} \subset J} 2^{-\nu n} \sup _{x \in Q_{\nu k}}|f(x)|^{q} \leq & C_{n, q}\left(\sum_{l \in \mathbb{Z}^{n}}(1+|l|)^{-(n+1)}\right) \\
& \cdot \sup _{\ell(J) \geq 2^{-\nu}} \sum_{Q_{\nu k} \subset J} \int_{Q_{\nu k}}|f(y)|^{q} d y .
\end{aligned}
$$


In the last step we have trivially shifted all cubes along the dyadic grid, which does not affect the supremum. Finally,

$$
\begin{aligned}
\sup _{\ell(J) \geq 2^{-\nu}} 2^{-\nu n p / q} \ell(J)^{n(q / p-1)} & \sum_{Q_{\nu k} \subset J}|Q|^{1-q / p} \sup _{x \in Q_{\nu k}}|f(x)|^{q} \\
& \leq \sup _{\ell(J) \geq 2^{\nu}} \ell(J)^{n(q / p-1)} \sum_{Q_{\nu k} \subset J} 2^{-\nu n} \sup _{x \in Q_{\nu k}}|f(x)|^{q} \\
& \leq C_{n, q} \sup _{\ell(J) \geq 2^{\nu}} \ell(J)^{n(q / p-1)} \int_{J}|f(y)|^{q} d y \\
& \leq C_{n, q}\|f\|_{\mathcal{M}_{q}^{p}}^{q} .
\end{aligned}
$$

If $f$ is any distribution, regardless of its support, replacing $f$ with $f_{\nu}$ in the above lemma easily gives

$$
\begin{aligned}
& {\left[\sum_{\nu \in \mathbb{Z}}\left(2^{\nu n(s / n-1 / p)}\left(\sup _{\ell(J) \geq 2^{-\nu}}\left(\frac{1}{|J|}\right)^{1-q / p} \sum_{Q_{\nu k} \subset J} 2^{-\nu n} \sup _{x \in Q_{\nu k}}\left|f_{\nu}(x)\right|^{q}\right)^{1 / q}\right)^{r}\right]^{1 / r}} \\
& \leq C\left[\sum_{\nu \in \mathbb{Z}}\left(2^{s \nu}\left\|\sigma_{\nu} * f\right\|_{\left.\mathcal{M}_{q}^{p}\right)^{r}}\right]^{1 / r}=C\|f\|_{\mathcal{N}_{p, q, r}^{s}},\right.
\end{aligned}
$$

for all $s \in \mathbb{R}, 1 \leq q \leq p<\infty, r \in[1, \infty]$.

Following [FJ1, let us introduce the following suggestive notation.

Notation 2.12. If $Q=Q_{\nu k}$, then set

$$
\begin{aligned}
\sigma_{Q} & =|Q|^{s / n-1 / p} \sigma_{\nu k}, \\
s_{Q} & =2^{\nu(s-n / p)} \sigma_{\nu} * f\left(2^{-\nu} k\right) .
\end{aligned}
$$

We can collect all previous results to obtain the following wavelet representation for the space $\mathcal{N}_{p, q, r}^{s}$.

Proposition 2.13. Let $f \in \mathcal{N}_{p, q, r}^{s}$. Then

$$
f=\sum_{Q \text { dyadic }} s_{Q} \sigma_{Q}
$$

in $\mathcal{S}^{\prime} / \mathcal{P}$, and

$$
C(n, p, q, r)\|f\|_{\mathcal{N}_{p, q, r}^{s}} \geq\left[\sum_{\nu \in \mathbb{Z}}\left(\sup _{\ell(J) \geq 2^{-\nu}}\left(\frac{1}{|J|}\right)^{1-q / p} \sum_{Q_{\nu k} \subset J}|Q|^{1-q / p}\left|s_{Q}\right|^{q}\right)^{r / q}\right]^{1 / r} .
$$

Note there is no explicit dependence on the index $p$ and $s$ in this decomposition. It is, in fact, hidden in the definition of $\sigma_{Q}$ and $s_{Q}$. Also, for $p=q$, we correctly recover the decomposition for Besov spaces (see [FJ1]). 
Proof. The representation formula for $f$ follows directly from Lemma 2.9 As for (2.33), it is enough to notice that $\sum_{Q} s_{Q} \sigma_{Q}$ with $Q=Q_{\nu k}$ satisfies the hypotheses of Lemma 2.11 for $\nu$ fixed. Hence, (2.32) becomes

$$
\begin{gathered}
C\|f\|_{\mathcal{N}_{p, q, r}^{s}} \geq\left[\sum _ { \nu \in \mathbb { Z } } \left(2^{-\nu n(s / n-1 / p) q} \sup _{\ell(J) \geq 2^{-\nu}}\left(\frac{1}{|J|}\right)^{1-q / p} .\right.\right. \\
\left.\left.\cdot \sum_{Q_{\nu k} \subset J}|Q|^{1-q / p} \sup _{x \in Q_{\nu k}}\left|\sum_{Q_{\nu l}} s_{Q} \sigma_{Q}(x)\right|^{q}\right)^{r / q}\right]^{1 / r} \\
=\left[\sum_{\nu \in \mathbb{Z}}\left(\sup _{\ell(J) \geq 2^{-\nu}}\left(\frac{1}{|J|}\right)^{1-q / p} \sum_{Q_{\nu k} \subset J}|Q|^{1-q / p} \sup _{x \in Q_{\nu k}}\left|\sum_{Q_{\nu l}} s_{Q} \sigma_{\nu k}(x)\right|^{q}\right)^{r / q}\right]^{1 / r} .
\end{gathered}
$$

But the cubes $Q_{\nu k}$ are pairwise disjoint. So (2.26) easily implies that the largest contribution to the sum above is attained when $k=l$.

It is straightforward to prove analogous propositions for the inhomogeneous spaces $N_{p, q, r}^{s}$. First of all, replace the homogeneous Littlewood-Paley partition of unity $\left\{\psi_{\nu}\right\}, \nu \in \mathbb{Z}$, with the corresponding inhomogeneous one $\left\{\phi_{0}, \psi_{\nu}\right\}, \nu \geq 0$ (using the notation introduced in Section 2.1), and let $s_{k}=\check{\varphi}_{0} * f(k)$. Second, since we take the local Morrey space $M_{q}^{p}$ as base space, we must restrict dyadic cubes to those of length $\ell(Q) \leq 1$.

Proposition 2.14. Let $f \in N_{p, q, r}^{s}$. Then

$$
f=\sum_{k \in \mathbb{Z}^{n}} s_{k} \check{\varphi_{0}}(\cdot-k)+\sum_{\nu \geq 0} \sum_{k \in \mathbb{Z}} s_{Q_{\nu k}} \sigma_{Q_{\nu k}}
$$

in $\mathcal{S}^{\prime}$, and

$$
\begin{aligned}
C\|f\|_{N_{p, q, r}^{s}} \geq \sup _{k \in \mathbb{Z}^{n}}\left|s_{k}\right| & \\
& +\left[\sum_{\nu \geq 0}\left(\sup _{2^{-\nu} \leq \ell(J) \leq 1}\left(\frac{1}{|J|}\right)^{1-q / p} \sum_{Q_{\nu k} \subset J}|Q|^{1-q / p}\left|s_{Q}\right|^{q}\right)^{r / q}\right]^{1 / r}
\end{aligned}
$$

Remark 2.15. The reverse of inequality (2.33) (and similarly (2.34) ) can be shown to hold. If $\left\{s_{Q}\right\}$ is any sequence indexed by dyadic cubes, $f=\sum_{Q} s_{Q} \sigma_{Q}$ in the sense of distributions, and

$$
\|f\|_{*}=\left[\sum_{\nu \in \mathbb{Z}}\left(\sup _{\ell(J) \geq 2^{-\nu}}\left(\frac{1}{|J|}\right)^{1-q / p} \sum_{Q_{\nu k} \subset J}|Q|^{1-q / p}\left|s_{Q}\right|^{q}\right)^{r / q}\right]^{1 / r}<\infty,
$$

then $f \in \mathcal{N}_{p, q, r}^{s}$ with $\|f\|_{N_{p, q, r}^{s}} \sim\|f\|_{*}$. More generally, $\sigma_{Q}$ can be replaced by an $(s, p)$-molecule, as is the case for Besov spaces FJ1. We do not have to decompose molecules concentrated on dyadic cubes in atoms supported on cubes (cf. [Uch] and the decomposition for BMO), because cancellations are not crucial in this context. Details are provided in Maz2. 
The above construction should also be compared with a similar one for the space BMO, which follows from work of Uchiyama [Uch]. Using the above notation, we have

$$
\|f\|_{\text {BMO }} \approx \sup _{J \text { dyadic }}\left(\sum_{\nu=-\log _{2}(\ell(J))}^{+\infty} \frac{1}{|J|} \sum_{Q_{\nu k} \subset J} 2^{-\nu n} \sup _{Q_{\nu k}}\left|\sigma_{\nu} * f\right|^{2}\right)^{1 / 2},
$$

and it is then easy to see, for example, that $\mathcal{N}_{p, q, r}^{n / p} \subset$ BMO for $q \geq 2, r \leq 2$.

Frazier and Javerth have extended Uchiyama's result to the whole class of Triebel-Lizorkin spaces $\dot{F}_{\infty, r}^{s}, 1 \leq r<\infty, s \in \mathbb{R}[$ FJ2]. Their decomposition along with (2.33) allows us to compare the Besov-Morrey space $\mathcal{N}_{p, q, \infty}^{n / p-1}, p>n$, with $\mathrm{BMO}^{-1}$. We are particularly interested in the relation between these spaces in connection with well-posedness at the critical scaling for the Navier-Stokes equation. We refer to Section 5 for a more extensive discussion.

The space $\mathrm{BMO}^{-1}$ is the space of tempered distributions that can be written as divergence of a vector field with components in BMO. The norm in $\mathrm{BMO}^{-1}$ is given by

$$
\|f\|_{\mathrm{BMO}^{-1}} \approx \sup _{x \in \mathbb{R}^{n}} \sup _{R>0}\left(R^{-n} \int_{B(x, R)} \int_{0}^{R^{2}}\left|e^{t \Delta} f(y)\right|^{2} d t d y\right)^{1 / 2} .
$$

Using the characterization (2.57), which is proved in the Section 2.3, it is easy to see that

$$
\mathcal{N}_{p, q, \infty}^{n / p-1} \subset \mathrm{BMO}^{-1}, \quad q \geq 2, p>n
$$

However, for $q=1$,

$$
\mathcal{N}_{p, 1, \infty}^{n / p-1} \nsubseteq \mathrm{BMO}^{-1}
$$

(and similarly $\mathrm{BMO}^{-1} \nsubseteq \mathcal{N}_{p, 1, \infty}^{n / p-1}$ ).

2.3. Scaling properties: the spaces $B E^{\alpha}$. Recently, G. Karch introduced a large class of Banach spaces based on scaling properties of the heat semigroup, and used them to study certain semi-linear parabolic equations $[\mathrm{Kr}$. BM spaces belong to this class for a particular range of indices.

We begin again with some definitions and background notation.

Definition 2.16. Let $\left(E,\|\cdot\|_{E}\right)$ be a functional Banach space, that is, $\mathcal{S} \hookrightarrow E \hookrightarrow$ $\mathcal{S}^{\prime}$. Set $f_{\lambda}(x)=f(\lambda x), \forall \lambda>0$. The norm $\|\cdot\|_{E}$ has scaling degree equal to $\kappa$, if $\forall f \in E$ and $\forall \lambda>0, f_{\lambda} \in E$ and $\left\|f_{\lambda}\right\|_{E}=\lambda^{\kappa}\|f\|_{E}$.

We will denote a space with scaling degree $-n / p$ by $E_{p}$. Both the space $L^{p}$ and the (homogeneous) Morrey space $\mathcal{M}_{q}^{p}$ have scaling degree equal to $-n / p$. BM spaces have the same scaling degree as Besov spaces (namely $s-n / p$ ), while being strictly larger. This property will be exploited in studying differential equations. We implicitly assume that $E$ always has some scaling degree, which is true for all classical spaces.

Norm estimates for the heat semigroup can be easily obtained by rescaling arguments. 
Lemma $2.17([\overline{\mathrm{Kr}}])$. Suppose $e^{t_{0} \Delta}: E_{q} \rightarrow E_{p}$ is a bounded linear operator for some $t_{0}>0$. Then, there exists $C=C\left(p, q, t_{0}\right)>0$ such that

$$
\left\|e^{t \Delta} f\right\|_{E_{p}} \leq C t^{n(1 / p-1 / q) / 2}\|f\|_{E_{q}}, \quad \forall t>0, \quad \forall f \in E_{q} .
$$

Moreover, if $q>p$, then necessarily $E_{q}=\{0\}$.

In view of the above Lemma, one expects $t^{\alpha / 2}\left\|e^{t \Delta} f\right\|_{E_{p}}$ to be bounded for some $\alpha>0$, even if $f$ belongs to a weak space of distributions.

Definition 2.18. Given a functional Banach space E, define

$$
B E^{\alpha}=\left\{f \in \mathcal{S}^{\prime} \mid\|f\|_{B E^{\alpha}} \equiv \sup _{t>0}\left\{t^{\alpha / 2}\left\|e^{t \Delta} f\right\|_{E}\right\}<\infty\right\} .
$$

It is not difficult to see that $B E^{\alpha}=\{0\}$ for $\alpha<0$, while $E \subset B E^{0}$, although this space is not as interesting. Therefore, we will implicitly assume in the following that $\alpha>0$.

Lemma (2.17) implies that $E_{q} \subset B E_{p}^{\alpha} \forall q$ such that $n(1 / q-1 / p) / 2 \leq \alpha / 2$ if $e^{t \Delta}: E_{q} \rightarrow E_{p}$ is bounded for some $t>0$. In particular,

$$
\begin{aligned}
& L^{q} \subset B E^{\alpha} \text { for } E=L^{p} \text { if } 1 \leq q \leq p \leq \infty \text { and } \alpha=n(1 / q-1 / p), \\
& \mathcal{M}_{q_{1}}^{p_{1}} \subset B E^{\alpha} \text { for } E=\mathcal{M}_{q_{2}}^{p_{2}} \text { if } 1 \leq p_{1} \leq p_{2} \leq \infty \text { and } \alpha=n\left(1 / p_{1}-1 / p_{2}\right) .
\end{aligned}
$$

In the case $E=L^{p}$, the space $B E^{\alpha}$ is, in fact, the homogeneous Besov Space $\dot{B}_{p, \infty}^{-\alpha}$. This is a consequence of the following characterization of Besov spaces in terms of the Weierstrass semigroup (see [Trieb1], page 192):

Theorem 2.19. For every $f \in \mathcal{S}^{\prime}$, the following norms are equivalent:

$$
\begin{gathered}
\|f\|_{\dot{B}_{p, \infty}^{-\alpha},}, \\
\sup _{t \geq 0}\left\{t^{\alpha / 2}\left\|e^{t \Delta} f\right\|_{L^{q}}\right\} .
\end{gathered}
$$

Intuitively, Theorem 2.19 holds because the symbol of the operator $e^{t \Delta}$ and the symbol of $\varphi_{j}(D)$ have a similar behavior in frequency.

The spaces $B E^{\alpha}$ are complete if the class of underlying $E$ 's is appropriately restricted.

Definition 2.20. $\mathcal{K}$ denotes the class of functional Banach spaces $E$ such that $e^{t \Delta}: E \rightarrow L^{q}$ is bounded $\forall t>0$ for some $q \in[1, \infty]$.

For example, the Morrey spaces $\mathcal{M}_{q}^{p}$ have this property with $q=\infty$.

Lemma $2.21(\underline{\mathrm{Kr}})$. Assume $E_{p} \in \mathcal{K}$. Then $\left(B E_{p}^{\alpha},\|\cdot\|_{B E_{p}^{\alpha}}\right)$ is a Banach Space.

In fact, if $E_{p} \in \mathcal{K}$, by Lemma 2.17 $B E_{p}^{\alpha} \hookrightarrow \dot{B}_{q, \infty}^{\gamma}$, where $q \geq p$ and $\gamma=$ $-\alpha-n(1 / p-1 / q)$. So, a Cauchy sequence $\left\{f_{k}\right\}$ in $B E_{p}^{\alpha}$ converges to $f$ in $\dot{B}_{q, \infty}^{\gamma}$. Moreover, $\left\{e^{t \Delta} f_{k}\right\}$ is a Cauchy sequence in $E_{p}$ for $t>0$ and, therefore, converges to some $g(t)$ in $E_{p}$. Since the $f_{k}$ 's are uniformly bounded in $B E^{\alpha}$, we can conclude that $\|g(t)\|_{E_{p}}$ grows in $t$ at most as $t^{-\alpha / 2}$, as $t \rightarrow 0$. So, it is enough to show that $g(t)=e^{t \Delta} f$ in $\mathcal{S}^{\prime}$. But this is true by continuity of the heat semigroup in $\mathcal{S}^{\prime}$ and because $E_{p} \hookrightarrow \mathcal{S}^{\prime}$.

Note that, if $E$ has scaling degree $\kappa$, then $B E^{\alpha}$ has scaling degree $\kappa-\alpha$. So, estimates similar to (2.39) hold for certain values of $\alpha$.

In view of Theorem 2.19, we expect all $B E^{\alpha}$ spaces to be characterized in terms of a Littlewood-Paley partition of unity. 
Proposition 2.22. Let $E$ be a functional Banach space such that the norm has some scaling degree $\gamma>0$. Then, an equivalent norm in $B E^{\alpha}$ is given by

$$
\|f\|_{B E^{\alpha}}^{*}=\sup _{j \in \mathbb{Z}}\left\{2^{-j \alpha}\left\|\psi_{j}(D) f\right\|_{E}\right\} .
$$

For clarity, we split the proof into different lemmas.

Lemma 2.23. If $f \in B E^{\alpha}$, then $\psi_{j}(D) f \in E, \forall j \in \mathbb{Z}$, and there is a constant $C=C(E, \alpha)$ such that

$$
\sup _{j \in \mathbb{Z}}\left\{2^{-j \alpha}\left\|\psi_{j}(D) f\right\|_{E}\right\} \leq C\|f\|_{B E^{\alpha}} .
$$

Proof. Set $t=4^{-j}$. Since $\psi_{j}(\xi)$ is supported on the shell $D_{j}=\left\{\xi \in \mathbb{R}^{n} \mid 2^{j-1}<\right.$ $\left.|\xi|<2^{j+1}\right\}$

$$
\begin{aligned}
\left\|\psi_{j}(D) f\right\|_{E} & =\left\|\mathcal{F}^{-1}\left(\psi_{j}(\xi) \hat{f}(\xi)\right)\right\|_{E} \\
& \leq \tilde{C}\left\|\mathcal{F}^{-1}\left(e^{-t|\xi|^{2}} \psi_{j}(\xi) \hat{f}(\xi)\right)\right\|_{E},
\end{aligned}
$$

so that

$$
\begin{aligned}
2^{-j \alpha}\left\|\psi_{j}(D) f\right\|_{E} & \leq \tilde{C} t^{\alpha / 2}\left\|\check{\psi}_{j}\right\|_{L^{1}}\left\|\mathcal{F}^{-1} e^{-t|\xi|^{2}} \hat{f}(\xi)\right\|_{E} \\
& \leq C t^{\alpha / 2}\left\|\mathcal{F}^{-1} e^{-t|\xi|^{2}} \hat{f}(\xi)\right\|_{E} \leq C t^{\alpha / 2}\left\|e^{t \Delta} f\right\|_{E},
\end{aligned}
$$

by means of Remark 2.8

Lemma 2.24. If $f \in \mathcal{S}^{\prime}$ is such that $\sup _{j \in \mathbb{Z}}\left\{2^{-j \alpha}\left\|\psi_{j}(D) f\right\|_{E}\right\}<\infty$, then

$$
\sup _{j \in \mathbb{Z}}\left\{2^{-j \alpha}\left\|\varphi_{j}(D) f\right\|_{E}\right\} \approx \sup _{j \in \mathbb{Z}}\left\{2^{-j \alpha}\left\|\psi_{j}(D) f\right\|_{E}\right\} .
$$

Proof. It is enough to show that, for each fixed $k, \varphi_{k}(D) f=\sum_{j=-\infty}^{k} \psi_{j}(D) f$ in $E$. Let $P_{l}^{(k)}=\sum_{j=-l}^{k} \psi_{j}(D) f$. Set $m \geq l$ and consider

$$
\left\|P_{m}^{(k)}-P_{l}^{(k)}\right\|_{E} \leq C \sum_{j=-m}^{-l-1}\left\|\psi_{j}(D) f\right\|_{E} \leq C\left(\sum_{j=-m}^{-l-1} 2^{j \alpha}\right) \sup _{j \in \mathbb{Z}}\left\{2^{-j \alpha}\left\|\psi_{j}(D) f\right\|_{E}\right\},
$$

where the last term tends to 0 as $l, m \rightarrow \infty$ if $\alpha>0$. Hence,

$$
\begin{aligned}
2^{-k \alpha}\left\|\varphi_{k}(D) f\right\|_{E} & \leq 2^{-k \alpha} \sum_{j=-\infty}^{k}\left\|\psi_{j}(D) f\right\|_{E} \\
& \leq\left(\sum_{j=-\infty}^{k} 2^{(j-k) \alpha}\right) \sup _{j \in \mathbb{Z}}\left\{2^{-j \alpha}\left\|\psi_{j}(D) f\right\|_{E}\right\} \\
& =\left(\sum_{j=-\infty}^{0} 2^{j \alpha}\right) \sup _{j \in \mathbb{Z}}\left\{2^{-j \alpha}\left\|\psi_{j}(D) f\right\|_{E}\right\} \\
& \leq C \sup _{j \in \mathbb{Z}}\left\{2^{-j \alpha}\left\|\psi_{j}(D) f\right\|_{E}\right\} .
\end{aligned}
$$

The reverse inequality is obvious.

Note that this lemma would be clearly false if $\alpha$ were negative or zero. 
Lemma 2.25. Suppose that the norm of $E$ has scaling degree equal to some $\gamma>0$. Then,

$$
\|f\|_{B E^{\alpha}} \leq C \sup _{j \in \mathbb{Z}}\left\{2^{-j \alpha}\left\|\psi_{j}(D) f\right\|_{E}\right\} .
$$

Proof. By Lemma 2.24 it is enough to show

$$
\|f\|_{B E^{\alpha}} \leq C \sup _{j \in \mathbb{Z}}\left\{2^{-j \alpha}\left\|\varphi_{j}(D) f\right\|_{E}\right\} .
$$

We need to estimate $t^{\alpha / 2}\left\|e^{t \Delta} f\right\|_{E}$. Using homogeneity of the norm, one can restrict to the case $t=1$. First, the semigroup property of $e^{t \Delta}$ allows us to reduce easily to $t=4^{-k}$ for $k \in \mathbb{Z}$. Then, if we set $f_{\lambda}(x)=f(\lambda x)$ for $\lambda>0$, we have

$$
e^{t \Delta} f_{\frac{1}{\sqrt{t}}}(x)=\left(e^{\Delta} f\right)_{\frac{1}{\sqrt{t}}}(x),
$$

which follows simply by changing variables $\tilde{y}=\frac{y}{\sqrt{t}}$ in the convolution $p_{t} * f$.

Similarly,

$$
\begin{aligned}
\varphi_{j}(D) f_{\frac{1}{\sqrt{t}}}(x) & =2^{n j}\left(\check{\varphi}_{0}\left(2^{j} \cdot\right) * f_{\frac{1}{\sqrt{t}}}\right)(x) \\
& =2^{n j} \int_{\mathbb{R}^{n}} \check{\varphi}_{0}\left(2^{j}(x-y)\right) f\left(2^{k} y\right) d y \\
& =2^{n(j-k)} \int_{\mathbb{R}^{n}} \check{\varphi}_{0}\left(2^{j-k}\left(2^{k} x-\tilde{y}\right)\right) f(\tilde{y}) d \tilde{y} \\
& =2^{n(j-k)} \int_{\mathbb{R}^{n}} \check{\varphi}_{0}\left(2^{j-k}(x / \sqrt{t}-\tilde{y})\right) f(\tilde{y}) d \tilde{y}=\left(\varphi_{(j-k)}(D) f\right)_{\frac{1}{\sqrt{t}}}(x) .
\end{aligned}
$$

So, replacing $f$ by $f_{\frac{1}{\sqrt{t}}}$ in (2.49) and setting $t=4^{-k}$ gives

$$
\begin{aligned}
t^{\alpha / 2}\left\|e^{t \Delta} f_{\frac{1}{\sqrt{t}}}\right\|_{E} & =t^{\alpha / 2}\left\|\left(e^{\Delta} f\right)_{\frac{1}{\sqrt{t}}}\right\|_{E} \\
& =t^{\alpha / 2} t^{-\gamma / 2}\left\|e^{\Delta} f\right\|_{E} \\
& =2^{k(\gamma-\alpha)}\left\|e^{\Delta} f\right\|_{E} \\
& \leq \sup _{j \in \mathbb{Z}}\left\{2^{-j \alpha}\left\|\varphi_{j}(D) f_{\frac{1}{\sqrt{t}}}\right\|_{E}\right\} \\
& =\sup _{j \in \mathbb{Z}}\left\{2^{-j \alpha}\left\|\left(\varphi_{(j-k)}(D) f\right)_{\frac{1}{\sqrt{t}}}\right\|_{E}\right\} \\
& =\sup _{j \in \mathbb{Z}}\left\{2^{-j \alpha} 2^{k \gamma}\left\|\varphi_{(j-k)}(D) f\right\|_{E}\right\} \\
& =\sup _{j \in \mathbb{Z}}\left\{2^{-(j-k) \alpha} 2^{k(\gamma-\alpha)}\left\|\varphi_{(j-k)}(D) f\right\|_{E}\right\},
\end{aligned}
$$

because $E$ has scaling degree $\gamma$.

By renaming indices $j-k \rightarrow j$, it remains to show that

$$
\left\|e^{\Delta} f\right\|_{E} \leq C \sup _{j \in \mathbb{Z}}\left\{2^{-j \alpha}\left\|\varphi_{j}(D) f\right\|_{E}\right\} .
$$

Now, on the left-hand side apply a Littlewood-Paley partition to $f$, so that it is more convenient to replace the supremum by a sum over $j$ on the right-hand side. 
To do so, fix $\beta$ and $\epsilon \geq 0$ such that $\beta-\epsilon>\alpha$. Then,

$$
\sum_{j \geq 0} 2^{-j \beta}\left\|\varphi_{j}(D) f\right\|_{E} \leq 2\left(\sum_{j \geq 0} 2^{-j \epsilon}\right) \sup _{k \in \mathbb{Z}}\left\{2^{-k \alpha}\left\|\varphi_{k}(D) f\right\|_{E}\right\} .
$$

Next, note that in $\mathcal{S}^{\prime}$,

$$
\begin{aligned}
\sum_{j \geq 0} 2^{-j \beta} \varphi_{j}(D) & =c \varphi_{0}(D)+\sum_{j \geq 0} 2^{-j \beta} \sum_{k=0}^{j} \psi_{k}(D) \\
& =c \varphi_{0}(D)+\sum_{k=0}^{\infty} \psi_{k}(D) \sum_{j=k}^{\infty} 2^{-j \beta} .
\end{aligned}
$$

But if $\Phi_{k}$ is a smooth compactly-supported function, which is identically one on the shell $D_{k}$, then the operator norm of the multiplier $e^{-|\xi|^{2}} \Phi_{k}(\xi)$ does not exceed $C_{\beta} 2^{-k \beta}$ for every $\beta \geq 0$, where $C_{\beta}$ does not depend on $k$ (cf. Proposition 3.3). Hence, (2.54) implies

$$
\begin{aligned}
\left\|e^{\Delta} f\right\|_{E} & \leq\left\|e^{\Delta} \varphi_{0}(D) f\right\|_{E}+\left\|e^{\Delta} \sum_{j \geq 0} \psi_{j}(D) f\right\|_{E} \\
& \leq C_{\beta} \sum_{j \geq 0} 2^{-j \beta}\left\|\varphi_{j}(D) f\right\|_{E}, \quad \forall \beta .
\end{aligned}
$$

Equivalence of norms such as (2.43) are very well-known in the theory of function spaces, where they are a consequence of interpolation relations between semigroups of operators and their infinitesimal generators BL, [Trieb2]. However, such results require that the semigroup be strongly continuous or even analytic. Our proof, instead, is based solely on some simple scaling arguments and requires the semigroup only to be uniformly bounded. Note, in particular, that the heat semigroup is not strongly continuous on Morrey spaces.

Consider now the homogeneous BM space $\mathcal{N}_{p, q, \infty}^{-\alpha}, \alpha>0$. By virtue of Proposition 2.5, it can be canonically identified with the set of distributions

$$
\left\{f \in \mathcal{S}^{\prime} \mid \sup _{j \in \mathbb{Z}}\left\{2^{-j \alpha}\left\|\Delta_{j} f\right\|_{E}\right\}<\infty\right\} .
$$

Therefore, Proposition 2.22 yields immediately

$$
\mathcal{N}_{p, q, \infty}^{-\alpha}=B E^{\alpha}
$$

for $E=\mathcal{M}_{q}^{p}$. Note that $\mathcal{M}_{q}^{p}$ has scaling degree $-n / p$; so Proposition 2.22 does indeed apply. In turn, this identification gives an equivalent norm in the space $\mathcal{N}_{p, q, \infty}^{s}, s<0$, which is useful when dealing with semi-linear parabolic equations:

$$
\|f\|_{\mathcal{N}_{p, q, \infty}^{s}} \approx \sup _{t>0}\left\{t^{-s / 2}\left\|e^{t \Delta} f\right\|_{\mathcal{M}_{q}^{p}}\right\} .
$$

Similar results hold for the local space $N_{p, q, r}^{s}$.

Proposition 2.26. Let $1 \leq q \leq p<\infty, s<0$. Then

$$
\|f\|_{N_{p, q, \infty}^{s}} \approx \sup _{0<t \leq 1}\left\{t^{-s / 2}\left\|e^{t \Delta} f\right\|_{M_{q}^{p}}\right\} .
$$

\footnotetext{
${ }^{5}$ This identification gives, in fact, a positive answer to a question of G. Karch [ $\left.\mathrm{Kr}\right]$.
} 
The proof is actually simpler, since no low-frequency part has to be considered.

Remark 2.27. Using the inclusion $N_{p, q, r}^{s} \subset N_{p, q, \infty}^{s}$ for $1 \leq r<\infty$, we immediately conclude that, if $s<0$,

$$
f \in N_{p, q, r}^{s} \Rightarrow \sup _{0<t \leq 1}\left\{t^{-s / 2}\left\|e^{t \Delta} f\right\|_{M_{q}^{p}}\right\}<\infty .
$$

Note that (2.59) is a bit stronger than norm estimates obtained by a simple rescaling argument, which would read:

$$
\|f\|_{N_{p, q, \infty}^{0}} \leq C t^{s / 2}\|f\|_{N_{p, q, r}^{s}} .
$$

\section{Pseudo-Differential Calculus}

Our focus in this section is to study pseudo-differential operators in the function spaces previously introduced. Pseudo-differential calculus is a well-established tool for the analysis of partial differential equations, especially non-linear ones. Our results improve decisively upon those in $[\mathrm{Kr}]$ and $[\mathrm{KY}]$. In particular, the theory developed here will be used extensively to define spaces on manifolds in Section 4

We will be mostly concerned with inhomogeneous spaces. Little can be said for the spaces $B E^{\alpha}\left(\mathcal{N}_{p, q, r}^{s}\right.$ in particular), unless the symbol definition is appropriately modified. Intuitively, the reason is the essentially local (or pseudo-local) nature of the operators we study. Their kernel can be highly singular along the diagonal, but it is smooth and rapidly decreasing away from it.

3.1. Basic estimates. For the sake of clarity, let us recall some basic definitions. A rather complete, but concise, treatment of the theory of pseudo-differential operators is given in Tay1. A classic, thorough reference is Tay4.

Given a function $p(x, \xi)$ on $\mathbb{R}^{n} \times \mathbb{R}^{n}$, one can define an operator $P$, acting say on $\mathcal{S}$ (if $p$ does not grow too fast at infinity), by

$$
P f(x)=\int_{\mathbb{R}^{n}} p(x, \xi) \hat{f}(\xi) e^{i x \cdot \xi} d \xi .
$$

The function $p$ is called the symbol, $\sigma(P)$, of the operator $P$. Fourier multipliers correspond to taking functions of $\xi$ alone.

We will also use the standard notation $p(x, D)$ for the operator $P$ with the function $p$ as symbol.

Definition 3.1. A smooth function $p(x, \xi)$ belongs to the symbol class $S_{\rho, \delta}^{m}, m \in \mathbb{R}$, $\rho, \delta \in[0,1]$, if it satisfies

$$
\left|D_{\xi}^{\alpha} D_{x}^{\beta} p(x, \xi)\right| \leq C_{\alpha, \beta}\langle\xi\rangle^{m-|\alpha| \rho+|\beta| \delta}
$$

for all multi-indices $\alpha$ and $\beta$. A pseudo-differential operator $P$ belongs to the class $O P S_{\rho, \delta}^{m}$ if its symbol is a function in $S_{\rho, \delta}^{m}$.

In $(\underline{3.2}),\langle\xi\rangle$ stands for $\left(1+|\xi|^{2}\right)^{1 / 2}$. The number $m$ is the order of the operator, while $(\rho, \delta)$ is its type.

Example 3.2. Let $P=\sum_{|\alpha|=0}^{m} a_{\alpha}(x) \partial_{1}^{\alpha_{1}} \cdots \partial_{n}^{\alpha_{n}}$ be any $m$-th order differential operator. Then, $P \in O P S_{1,0}^{m}$ and $\sigma(P)=\sum_{|\alpha|=0}^{m} a_{\alpha}(x) \xi_{1}^{\alpha_{1}} \cdots \xi_{n}^{\alpha_{n}}$. 
The symbol class $S_{\rho, \delta}^{m}$ was first introduced by Hörmander [Ho]. Operators in this class behave nicely on Sobolev spaces, namely $P: H^{s} \rightarrow H^{s-m}$ for $P \in S_{\rho, \delta}^{m}$ with $0 \leq \delta<\rho \leq 1$. Also, as long as $\delta<1, P: \mathcal{S}^{\prime} \rightarrow \mathcal{S}^{\prime}$.

Under the above hypotheses, composition and taking adjoints are well-defined operations; precisely,

(1) $P \in O P S_{\rho, \delta}^{m}, 0 \leq \delta<\rho \leq 1 \Rightarrow P^{*} \in O P S_{\rho, \delta}^{m}$,

(2) $P_{j} \in O P S_{\rho_{j}, \delta_{j}}^{m_{j}}, j=1,2 \Rightarrow P_{1} \circ P_{2} \in O P S_{\rho, \delta}^{m}$ with $\rho=\min \left(\rho_{1}, \rho_{2}\right)$ and $\delta=\max \left(\delta_{1}, \delta_{2}\right)$ if $0 \leq \delta_{2}<\rho \leq 1$.

Finally, it is customary to set

$$
O P S^{-\infty}=\bigcap_{\substack{m \in \mathbb{R} \\ \rho, \delta}} O P S_{\rho, \delta \in[0,1]}^{m} .
$$

An operator $P \in O P S^{-\infty}$ is called a smoothing operator, because

$$
\begin{aligned}
& P: \mathcal{S}^{\prime} \rightarrow C^{\infty}, \text { and } \\
& P: \mathcal{E}^{\prime} \rightarrow \mathcal{S} .
\end{aligned}
$$

Many other definitions and generalizations of symbol classes are clearly possible, and we will consider some later in the section. For the applications we have in mind, it is enough to take $\rho=1$. On the other hand, $\delta$ will range over the whole interval $[0,1]$.

We now turn to establishing mapping properties of pseudo-differential operators for $\mathrm{BM}$ and $B E^{\alpha}$ spaces. Since the symbol of the Stokes operator (on $\mathbb{R}^{n}$ ) has constant coefficients, the results found in $[\mathrm{KY}]$ and $[\mathrm{Kr}]$ concern the behavior of Fourier multipliers. Let $\left\{\psi_{j}\right\}$ be the usual (homogeneous) Littlewood-Paley partition of unity subordinated to the dyadic decomposition of $\mathbb{R}^{n}$ into the shells $D_{j}$.

Proposition 3.3 ([KY]). Let $m \in \mathbb{R}$ and $j \in \mathbb{Z}$. If $P(\xi)$ is a $C^{\infty}$ function on $D_{j-1} \cup D_{j} \cup D_{j+1}=\tilde{D}_{j}$ such that $P$ satisfies

$$
\left|\partial^{|\alpha|} P / \partial \xi^{\alpha}\right| \leq A 2^{(m-|\alpha|) j},
$$

for $\xi \in \tilde{D}_{j}$ and some constant $A, \forall \alpha \in \mathbb{N}^{n}$ such that $|\alpha| \leq[n / 2]+1$, then there exists $C>0$ with the following property: $\forall u \in M_{q}^{p}$ (respectively $\mathcal{M}_{q}^{p}$ ), $1 \leq q \leq p<\infty$, such that $\operatorname{Supp} \mathcal{F} u \subset D_{j}$, we have $P(D) u \in M_{q}^{p}$ (respectively $\mathcal{M}_{q}^{p}$ ) and

$$
\|P(D) u\|_{M_{q}^{p}} \leq C A 2^{m j}\|u\|_{M_{q}^{p}}
$$

(with a similar estimate for $\mathcal{M}_{q}^{p}$ ).

It is enough to establish the following bound on the Schwartz kernel of $P(D) \Phi(D)$, $K(x-y)$ :

$$
\|K\|_{L^{1}} \leq C A 2^{m j},
$$

which is an easy consequence of (3.4) and the Plancherel theorem. Here, $\Phi$ is a function in $C_{0}^{\infty}$ that is identically one on $\tilde{D}_{j}$.

Proposition 3.3 readily gives continuity of Fourier multipliers in BM spaces. 
Proposition 3.4 ([KY] $)$. Let $m, s \in \mathbb{R}, 1 \leq q \leq p<\infty, r \in[1, \infty]$. Let $N \geq[n / 2]+1$.

a) If $P(\xi)$ is a $C^{N}$ function on $\mathbb{R}^{n} \backslash\{0\}$ such that

$$
\left|\partial^{|\alpha|} P / \partial \xi^{\alpha}\right| \leq A|\xi|^{(m-|\alpha|)}, \quad \forall|\alpha| \leq N
$$

then

$$
P(D): \mathcal{N}_{p, q, r}^{s} \rightarrow \mathcal{N}_{p, q, r}^{s-m}
$$

is a bounded linear operator.

b) If $P(\xi)$ is a $C^{N}$ function on $\mathbb{R}^{n}$ such that

$$
\left|\partial^{|\alpha|} P / \partial \xi^{\alpha}\right| \leq A\langle\xi\rangle^{(m-|\alpha|)}, \quad \forall|\alpha| \leq N
$$

then

$$
P(D): N_{p, q, r}^{s} \rightarrow N_{p, q, r}^{s-m}
$$

is a bounded linear operator.

Note that if $P(\xi) \in S_{1,0}^{m}$, then it satisfies (3.7) for every $N$. Similarly, following Tay3, we define the symbol class $\Sigma_{1}^{m}$ to be the set of smooth functions on $\mathbb{R}^{n} \backslash\{0\}$ such that (3.6) holds for every $N$. Note that $S_{1,0}^{m} \subset \Sigma_{1}^{m}$ if $m \leq 0$, but if $m>0$, condition (3.6) is stronger than (3.7), at least when $|\alpha|<m$.

It is straightforward to extend these results to the whole class $B E^{\alpha}$, since they are essentially a consequence of invariance under translation for the norm of the base space.

Notation 3.5. To avoid confusion with the multi-index $\alpha$ in the definition of symbol classes, in this section we will denote $B E^{\alpha}$ by $B E^{-s}, s<0$.

We restrict $E$ to the class $\mathcal{K}$ (see Section 2.3) and assume the norm has scaling degree $-n / p$, so that $B E^{-s}$ is a Banach space. This is not a severe restriction. In particular, it is satisfied by $L^{p}$ and $\mathcal{M}_{q}^{p}$. The analogue of Proposition 3.3 holds word by word if $\mathcal{M}_{q}^{p}$ is replaced by $E$ :

Lemma 3.6. Let $m \in \mathbb{R}$ and $j \in \mathbb{Z}$. If $P(\xi)$ is a $C^{\infty}$ function on $D_{j-1} \cup D_{j} \cup D_{j+1}=$ $\tilde{D}_{j}$ such that $P$ satisfies

$$
\left|\partial^{|\alpha|} P / \partial \xi^{\alpha}\right| \leq A 2^{(m-|\alpha|) j},
$$

for $\xi \in \tilde{D}_{j}$ and some constant $A, \forall \alpha \in \mathbb{N}^{n}$ such that $|\alpha| \leq[n / 2]+1$, then there exists $C>0$ with the following property: $\forall u \in E$ such that $\operatorname{Supp} \mathcal{F} u \subset D_{j}$, we have $P(D) u \in E$ and

$$
\|P(D) u\|_{E} \leq C A 2^{m j}\|u\|_{E} .
$$

Proposition 3.7. Let $m \in \mathbb{R}$. Suppose $s<0$ and $s<m$. Let $N \geq[n / 2]+1$. If $P(\xi)$ is a $C^{N}$ function on $\mathbb{R}^{n} \backslash\{0\}$ such that

$$
\left|\partial^{|\alpha|} P / \partial \xi^{\alpha}\right| \leq A|\xi|^{(m-|\alpha|)}, \quad \forall|\alpha| \leq N,
$$

then

$$
P(D): B E^{-s} \rightarrow B E^{m-s}
$$

is a bounded linear operator. 
Proof. Recall that an equivalent norm in $B E^{m-s}$, if $m-s>0$, is given by

$$
\|u\|_{B E^{m-s}} \approx \sup _{j \in \mathbb{Z}}\left\{2^{-j(m-s)}\left\|\psi_{j}(D) u\right\|_{E}\right\} .
$$

It is therefore enough to prove that

$$
\left\|\psi_{j}(D) P(D) u\right\|_{E} \leq C 2^{m j}\left\|\psi_{j}(D) u\right\|_{E}
$$

where $P(D)$ and $\psi_{j}(D)$ commute, as scalar Fourier multipliers. Hence, we apply Lemma 3.6 with $u$ replaced by $u_{j}=\psi_{j}(D) u$ and $P(D)$ by $P(D) \Phi(D)$. On the shell $D_{j},|\xi| \sim 2^{j}$, so that $P(\xi) \Phi(\xi)$ satisfies (3.8).

The spaces $B E^{\alpha}$ do not form a scale. Consequently, operators of negative index do not have the usual smoothing effect. In the following sections we will concentrate on inhomogeneous BM spaces, which do form a scale.

Remark 3.8. The identification given by Proposition 2.22 breaks down if $s>0$, since then $B E^{-s} \equiv\{0\}$, while the spaces defined as

$$
\left\{f \in \mathcal{S}^{\prime} / \mathcal{P} \mid \sup _{j \in \mathbb{Z}}\left\{2^{s j}\left\|\psi_{j}(D) f\right\|_{E}\right\}<\infty\right\}, \quad s \in \mathbb{R},
$$

are non-trivial even if $s>0$ (take $E=L^{p}$, which gives the classical homogeneous Besov spaces). The proof above still holds for these spaces when $s>m$, but they are not spaces of distributions in general.

3.2. Further results: non-regular symbols. Now we consider general pseudodifferential operators $p(x, D)$. The symbol class $S_{1, \delta}^{m}$ is a little bit too restrictive for applications to non-linear equations, where typically differential operators have coefficients that depend on the solution itself. Therefore, while their symbol is still a smooth function of $\xi$, in general it will have only limited regularity in $x$.

We will concern ourselves with symbols for which $x$-regularity is measured in Hölder or Zygmund spaces. Other scales of spaces can be used [Tay2]. Our choice is motivated especially by Theorem 2.4 in connection with para-differential calculus (cf. Section 3.3).

Definition 3.9. The function $p(x, \xi)$ on $\mathbb{R}^{n} \times \mathbb{R}^{n}$ belongs to the symbol class $C_{*}^{\ell} S_{1, \delta}^{m}$, $\delta \in[0,1], \ell>0$, if it is smooth in $\xi$ and satisfies the following estimates:

$$
\begin{aligned}
\left\|\partial^{\alpha} p(\cdot, \xi) / \partial \xi^{\alpha}\right\|_{C_{*}^{\ell}} & \leq A_{\alpha}\langle\xi\rangle^{(m-|\alpha|+\ell \delta)} \text { and } \\
\left|\partial^{\alpha} p(x, \xi) / \partial \xi^{\alpha}\right| & \leq C_{\alpha}\langle\xi\rangle^{(m-|\alpha|)} .
\end{aligned}
$$

Similarly, one defines the class $C^{\ell} S_{1, \delta}^{m}, \delta \in[0,1], \ell>0$, as the collection of symbols $p(x, \xi)$ such that

$$
\begin{aligned}
\left\|\partial^{\alpha} p(\cdot, \xi) / \partial \xi^{\alpha}\right\|_{C^{\ell}} & \leq A_{\alpha}\langle\xi\rangle^{(m-|\alpha|+\ell \delta)} \quad \text { and } \\
\left|\partial^{\alpha} p(x, \xi) / \partial \xi^{\alpha}\right| & \leq C_{\alpha}\langle\xi\rangle^{(m-|\alpha|)} .
\end{aligned}
$$

The differences between the two classes are minor, especially if $\ell \in \mathbb{R}_{+} \backslash \mathbb{Z}$.

Notation 3.10. If $X$ is a function space, we will write $p(x, D) \in X O P S_{1, \delta}^{m}$ if $p(x, \xi)$ satisfies (3.12) with $\|\cdot\|_{X}$ in place of the $C_{*}^{\ell}$-norm.

Propositions 3.4 or 3.7 do not immediately extend to pseudo-differential operators, in particular, because $p(x, D)$ and $\psi_{j}(D)$ do not commute. Since $\psi_{j}(D)$ is 
scalar, the commutator $Q=\left[p(x, D), \psi_{j}(D)\right]$ will have lower order. However, it will not be a smoothing operator, even if $p(x, \xi)$ is a regular symbol.

However, if $p(x, \xi) \in S_{1, \delta}^{m}, \delta<1$, there is an asymptotic expansion for the symbol of $Q$ :

$$
q(x, \xi) \sim-\sum_{|\beta|>0} \frac{i^{|\beta|}}{\beta !} D_{\xi}^{\beta} \psi_{j}(\xi) D_{x}^{\beta} p(x, \xi),
$$

and $q(x, \xi)$ resembles a so-called elementary symbol (see, for example, Bour2 or CM]).

The asymptotic relation $\sim$ is given as follows. If $q$ is a symbol in $S_{1, \delta}^{m}$, then $q \sim \sum_{j \geq 0} p_{j}$ if, for each $k>0, q-\sum_{j \leq k} p_{j} \in S_{1, \delta}^{m-k}$. In other words, $\sum_{j} p_{j}(x, D)$ converges to $q(x, D)$ modulo smoothings. $p_{0}$ is called the principal symbol of $q(x, D)$, also denoted with $\sigma_{0}(q)$. It is customary to refer to $\sum_{j} p_{j}$ as "the" asymptotic expansion of $q$; although, clearly only the principal symbol is uniquely defined.

If $p \sim \sum_{j} p_{j}$ and each $p_{j}$ is a smooth function that is homogeneous of degree $m-j$ for $\xi$ large, then $p$ is called a classical symbol of order $m$, and we write $p \in S_{\mathrm{cl}}^{m}$.

Definition 3.11. We call elementary symbol in the class $C_{*}^{\ell} S_{1, \delta}^{m}, 0 \leq \delta \leq 1$, an expression of the form

$$
\sigma(x, \xi)=\sum_{j \geq 0} \sigma_{j}(x) \psi_{j}(\xi),
$$

where $\psi_{0}$ is smooth supported on the ball $B(0,2), \psi_{j}(\xi)=\psi\left(\xi / 2^{j}\right)$ and $\psi \in C_{0}^{\infty}$ is supported on the dyadic shell $D_{0}=\left\{\xi \in \mathbb{R}^{n}|1 / 2 \leq| \xi \mid \leq 2\right\}$, while $\left\{\sigma_{j}\right\}$ is a uniformly bounded sequence such that

$$
\left\|\sigma_{j}\right\|_{C_{*}^{\ell}} \leq C 2^{j(m+\ell \delta)} .
$$

Convergence is intended in the Fréchet topology of $C_{*}^{\ell} S_{1, \delta}^{m}$ induced by the seminorms

$$
\begin{aligned}
& \pi_{N}(\sigma)=\sup _{\substack{\xi \in \mathbb{R}^{n} \\
|\alpha| \leq N}}\langle\xi\rangle^{\alpha-(\ell \delta+m)}\left\|\partial_{\xi}^{\alpha} \sigma(\cdot, \xi)\right\|_{C_{*}^{\ell}}, \\
& \tilde{\pi}_{N}(\sigma)=\sup _{\substack{\xi \in \mathbb{R}^{n} \\
|\alpha| \leq N}}\langle\xi\rangle^{\alpha-m}\left\|\partial_{\xi}^{\alpha} \sigma(\cdot, \xi)\right\|_{L^{\infty}} .
\end{aligned}
$$

Example 3.12. If $\left\{\psi_{j}\right\}$ is a Littlewood-Paley partition of unity and $\left\{\sigma_{j}\right\}$ is a sequence uniformly bounded in $C_{*}^{\ell}\left(\mathbb{R}^{n}\right)$, then

$$
\sigma(x, \xi)=\sum_{j \geq 0} \sigma_{j}\left(2^{j \delta} x\right) \psi_{j}(\xi)
$$

is an elementary symbol in $C_{*}^{\ell} S_{1, \delta}^{0}$.

It is well-known ( Bour, , Ma $)$ that every symbol $\sigma$ can be decomposed into a rapidly convergent sum of elementary symbols,

$$
\sigma=\sum_{k \in \mathbb{Z}^{n}} \sigma_{k}
$$

\footnotetext{
${ }^{6}$ Here $\psi_{0}$ corresponds to $\varphi_{0}$ in our previous notation.
} 
where $\sigma_{k}$ is such that $\pi_{N}\left(\sigma_{k}\right) \leq C \pi_{N}(\sigma)$. Informally, such a decomposition is obtained by first applying a Littlewood-Paley partition of unity and then expanding each component in an orthonormal basis of $L^{2}\left(\mathbb{R}_{\xi}^{n}\right)$ to separate the $x$ and $\xi$ dependence.

Therefore, the operator with symbol $\sigma$ can be resolved into "elementary operators" with symbols $\sigma_{k}$, which justifies restricting to operators with elementary symbols. Thus, whenever the norm of target and source spaces is defined itself in terms of Littlewood-Paley components, the decomposition of the symbol and the decomposition of the function can be "matched" and the problem is reduced to bound localized objects.

This idea has been exploited to establish continuity of pseudo-differential operators with non-regular symbols in inhomogeneous Sobolev spaces $H^{s, p}$ and Zygmund spaces $C_{*}^{s} \mathrm{Ma}$, [Ru2], [Bour]. Here $\delta$ can be equal to 1 ; so the "exotic" Hörmander class $L_{1,1}$ is included.

However, the proof breaks down for the spaces $C_{*}^{s}$, when $s \leq 0$. Indeed, a crucial step is given by the following lemma (see [Tay1], for example).

Lemma 3.13. Let $f=\sum_{j \geq 0} f_{j}$ in $\mathcal{S}^{\prime}$, with $\operatorname{Supp} \hat{f}_{j} \subset B\left(0, A 2^{j}\right)$ for some $A>0$. Then, for $s>0$,

$$
\|f\|_{C_{*}^{s}} \leq C(A) \sup _{j \geq 0}\left\{2^{j s}\left\|f_{j}\right\|_{L^{\infty}}\right\} .
$$

The restriction on the range of $s$ cannot be lifted, since the hypotheses imply that the support of each Littlewood-Paley function $\psi_{j}$ intersects the support of infinitely many $f_{k}$. But each $\left\|f_{k}\right\|_{L^{\infty}}$ is weighted by a factor of $2^{-k s}$. So the total contribution is divergent for $s \leq 0$ (cf. proof of Lemma 3.16). For Sobolev spaces, duality and interpolation can be used instead to extend the analysis to the case $s<0$.

Recall that the spaces $B E^{-s}$ correspond to Besov-type spaces of negative index. So the above proof cannot be adapted in this context. On the other hand, even for the classical (homogeneous) Besov spaces, there is no analog to the lemma above if $s$ is positive large enough.

Clearly, there is no restriction on $s$ if the $f_{k}$ 's are supported on dyadic shells instead of balls (cf. Lemma 3.16). But then, to use the same approach, one is forced to consider symbols modeled on the class $\Sigma_{1}^{m}$. Due to the possible singularity of the symbol at the origin, convergence issues arise and there is no good calculus.

For the homogeneous BM spaces, one could try and exploit the representation of $\mathcal{N}_{p, q, r}^{s}$ as real interpolation spaces (see Proposition 2.6). However, the spaces $\mathcal{N}_{p, q, r}^{s}$ are actually better behaved than the spaces $(-\Delta)^{s / 2} \mathcal{M}_{q}^{p}$. For example, the former are closed under the action of Fourier multipliers of the Mikhlin-Hörmander type while the latter are not (for $q=1$ ) Tay3], Tay6]. Indeed, in general such symbols are not even bounded in $L^{1}$.

Hence, from now on we will concentrate on local spaces. As expected, the theory is similar to that for Besov and Zygmund spaces [Ru1], [Ru2], Bour, [Ma].

First of all, there are better interpolation results, since one uses real interpolation on the scale of spaces $(I-\Delta)^{-s / 2} M_{q}^{p}$. From Proposition 2.6,

$$
\left((I-\Delta)^{-s_{1} / 2} M_{q}^{p},(I-\Delta)^{-s_{2} / 2} M_{q}^{p}\right)_{\theta, r}=N_{p, q, r}^{s},
$$

\footnotetext{
${ }^{7} L_{1, \delta}$ is another standard notation for $O P S_{1, \delta}^{0}$.
} 
where $\theta \in(0,1)$ and $s=(1-\theta) s_{1}+\theta s_{2}$. Therefore, a linear operator $P$ bounded on $(I-\Delta)^{-s_{i} / 2} M_{q}^{p}$ for $i=1,2$ is also bounded on $N_{p, q, r}^{s}$ ( [Trieb2], p. 63). A first partial result follows easily.

Proposition 3.14. If $P \in O P S_{1, \delta}^{m}, m \in \mathbb{R}, \delta<1$, then

$$
P: N_{p, q, r}^{s} \rightarrow N_{p, q, r}^{s-m}, \quad \forall s \in \mathbb{R}, r \in[1, \infty], 1<q \leq p<\infty .
$$

Proof. Since operators in $O P S_{1, \delta}^{m}, \delta<1$, compose well, we can reduce to the case $m=0 . P$ is bounded in $M_{q}^{p}$ for $1<q \leq p<\infty$ Tay3. But $\sigma(P) \in S_{1, \delta}^{0}$ implies $(I-\Delta)^{s / 2} P(I-\Delta)^{-s / 2} \in O P S_{1, \delta}^{0}$.

To treat non-regular symbols, which do not have good multiplicative properties, we use elementary symbols and follow the approach in Bour and Ma]. Again, the idea is to compare the decomposition in frequency of the symbol with that of the function on which the symbol acts. To do so, we need a couple of preliminary lemmas. For the reader's convenience we give proofs, although they are very similar to that of Lemma 3.13 .

Lemma 3.15. Let $\left\{f_{k}\right\}, k \geq 0$, be a sequence of tempered distributions such that for some $A>0$, Supp $\hat{f}_{0} \subset B(0, A 2)$ and $\operatorname{Supp} \hat{f}_{k} \subset\left\{\xi \in \mathbb{R}^{n}\left|A 2^{k-1}<\right| \xi \mid<A 2^{k+1}\right\}$ for $k>0$. Then

$$
\left\|\sum_{k} f_{k}\right\|_{N_{p, q, r}^{s}} \leq C(A)\left(\left\|f_{0}\right\|_{M_{q}^{p}}+\|\left\{2^{s k}\left\|f_{k}\right\|_{\left.M_{q}^{p}\right\}_{k=1}^{\infty}} \|_{\ell^{r}}\right) .\right.
$$

Proof. Let $\left\{\varphi_{0}, \psi_{j}\right\}$ be the usual (inhomogeneous) Littlewood-Paley partition of unity. By hypothesis, $\psi_{j}$ is supported on the dyadic shell $D_{j}$, while $\varphi_{0}$ is supported on the ball $B(0,2)$. Hence, there is an $N=N(A)$ independent of $k$ such that

$$
\begin{aligned}
& \psi_{j}(D)\left(\sum_{k} f_{k}\right)=\psi_{j}(D)\left(\sum_{k=j-N}^{j+N} f_{k}\right), \\
& \varphi_{0}(D)\left(\sum_{k} f_{k}\right)=\varphi_{0}(D)\left(\sum_{k=0}^{N} f_{k}\right) .
\end{aligned}
$$

Consequently,

$$
\begin{aligned}
\left\|\sum_{k} f_{k}\right\|_{N_{p, q, r}^{s}} & =\left\|\varphi_{0}(D)\left(\sum_{k=0}^{N} f_{k}\right)\right\|_{M_{q}^{p}} \\
& +\left\{\sum_{j \geq 0}\left[2^{s j}\left\|\psi_{j}(D)\left(\sum_{k=j-N}^{j+N} f_{k}\right)\right\|_{M_{q}^{p}}\right]^{r}\right\}^{1 / r} \\
& \leq C \sum_{k=0}^{N}\left\|f_{k}\right\|_{M_{q}^{p}}+\left\{\sum_{j \geq N}\left(2^{s j} \sum_{k=j-N}^{j+N}\left\|f_{k}\right\|_{M_{q}^{p}}\right)^{r}\right\}^{1 / r},
\end{aligned}
$$


since $\left\{\varphi_{0}(D), \psi_{j}(D)\right\}$ is uniformly bounded in $O P S_{1,0}^{0}$. We estimate each part separately:

$$
\begin{aligned}
\sum_{k=0}^{N}\left\|f_{k}\right\|_{M_{q}^{p}} & \leq\left(\sum_{k=0}^{N} 2^{-j k r^{\prime}}\right)^{1 / r^{\prime}}\left\{\sum_{k \geq 0}\left(2^{k s}\left\|f_{k}\right\|_{M_{q}^{p}}\right\}^{r}\right\}^{1 / r} \\
& \leq C\left\{\sum_{k \geq 0}\left(2^{k s}\left\|f_{k}\right\|_{\left.M_{q}^{p}\right)^{r}}\right\}^{1 / r}\right.
\end{aligned}
$$

using Hölder's inequality with exponent $r^{\prime}$ conjugate to $r$. We then bound the remaining piece:

$$
\begin{gathered}
\left\{\sum_{j \geq N}\left(2^{s j} \sum_{k=j-N}^{j+N}\left\|f_{k}\right\|_{M_{q}^{p}}\right)^{r}\right\}^{1 / r} \\
=C\left\{\sum_{j \geq 0}\left[\sum_{l=0}^{2 N} 2^{-l s}\left(2^{(l+j) s}\left\|f_{l+j}\right\|_{\left.M_{q}^{p}\right)}\right]^{r}\right\}^{1 / r}\right. \\
\leq C \sum_{l=0}^{2 N} 2^{-l s}\left\{\sum_{k \geq 0}\left(2^{(l+k) s}\left\|f_{l+k}\right\|_{\left.M_{q}^{p}\right)^{r}}\right\}^{1 / r}\right. \\
\leq \tilde{C}\left\{\sum_{k \geq 0}\left(2^{k s}\left\|f_{k}\right\|_{M_{q}^{p}}\right)^{r}\right\}^{1 / r} .
\end{gathered}
$$

This is basically Proposition 2.8 in [KY], except that our proof works also for $r=\infty$ (all we used is the triangle and Hölder inequalities).

Lemma 3.16. Let $\left\{f_{k}\right\}, k \geq 0$, be a sequence of tempered distributions such that for some $A>0$, Supp $\hat{f}_{k} \subset B\left(0, A 2^{k+1}\right)$. Then for $s>0$,

$$
\left\|\sum_{k} f_{k}\right\|_{N_{p, q, r}^{s}} \leq C(A)\left\|\left\{2^{s k}\left\|f_{k}\right\|_{M_{q}^{p}}\right\}_{k \geq 0}\right\|_{\ell^{r}} .
$$

Proof. The low-frequency part is taken care of by the previous lemma. Concentrate on the high-frequency part. Again, in view of the hypothesis on $\operatorname{Supp} \psi_{j}$, there exists an $N=N(A)$ independent of $j$ such that

$$
\psi_{j}(D)\left(\sum_{k \geq 0} f_{k}\right)=\psi_{j}(D)\left(\sum_{k \geq j-N} f_{k}\right) .
$$


Hence, by the triangle inequality:

$$
\begin{aligned}
\left\|\sum_{k} f_{k}\right\|_{N_{p, q, r}^{s}} & \leq\left\|\left\{2^{s j}\left(\sum_{k \geq j-N}\left\|f_{k}\right\|_{M_{q}^{p}}\right)\right\}_{j \geq N}\right\|_{\ell^{r}} \\
& =\left\|\left\{2^{s j}\left(\sum_{l \geq-N} 2^{-(j+l) s} 2^{(j+l) s}\left\|f_{j+l}\right\|_{M_{q}^{p}}\right)\right\}_{j \geq N}\right\|_{\ell^{r}} \\
& \leq \sum_{l \geq-N} 2^{-l s} \|\left\{2^{(j+l) s}\left\|f_{j+l}\right\|_{\left.M_{q}^{p}\right\}_{j \geq N} \|_{\ell^{r}}}\right. \\
& \leq\left(\sum_{l \geq-N} 2^{-l s}\right) \|\left\{2^{j s}\left\|f_{j}\right\|_{\left.M_{q}^{p}\right\}_{j \geq 0} \|_{\ell^{r}}}\right. \\
& \leq C \|\left\{2^{j s}\left\|f_{j}\right\|_{\left.M_{q}^{p}\right\}_{j \geq 0} \|_{\ell^{r}},}\right.
\end{aligned}
$$

as long as $s>0$.

We are now ready to prove our main result.

Theorem 3.17. Let $p(x, \xi) \in C_{*}^{\ell} S_{1, \delta}^{m}$, where $m \in \mathbb{R}, \delta \in[0,1]$. Then

$$
p(x, D): N_{p, q, r}^{s+m} \rightarrow N_{p, q, r}^{s}
$$

if $0<s<\ell, 1 \leq q \leq p<\infty, r \in[1, \infty]$.

Remark 3.18. Note that $\delta$ can be equal to 1 . Therefore, the para-differential cas 8 is included.

The proof follows closely that for Sobolev Spaces Ma. First of all, by Proposition [3.4] it is enough to examine the case $m=0$. Indeed, $(I-\Delta)^{m / 2}$ is an isomorphism between $N_{p, q, r}^{s}$ and $N_{p, q, r}^{s+m}$ and the composition $p(x, D) \circ(I-\Delta)^{m / 2}$ is well-defined even for symbols with limited smoothness.

In addition, we are reduced to considering elementary symbols. This is possible, because for elementary operators, convergence in the operator norm of $\mathcal{L}\left(N_{p, q, r}^{s}\right)$ is equivalent to convergence of the symbols in the Fréchet topology of $C_{*}^{\ell} S_{1, \delta}^{0}$, which will be established in the course of the proof.

Proof. Let $q$ be an elementary symbol of the form

$$
q(x, \xi)=\sum_{j \geq 0} q_{j}(x) \psi_{j}(\xi)
$$

where $q_{j}$ satisfies

$$
\begin{aligned}
\left\|q_{j}\right\|_{C_{*}^{\ell}} & \leq C 2^{j \ell \delta}, \\
\left\|q_{j}\right\|_{L^{\infty}} & \leq C,
\end{aligned}
$$

with $C$ depending on $\delta$ and $\ell$ but not on $j$. By modifying $q_{j}$, if necessary, we can always assume $\psi_{j}$ is exactly a Littlewood-Paley function.

Set $\psi_{k}(D) q_{j}=q_{k j}$. Then, (3.29a) is equivalent to

$$
\left\|q_{k j}\right\|_{L^{\infty}} \leq C 2^{j \ell \delta} 2^{-k \ell} .
$$

${ }^{8}$ See Section 3.3 
Again, the idea is to "match" the decomposition of each $q_{j}$ into $q_{k j}$ with the de-

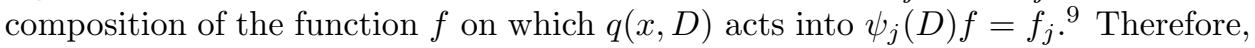
we rewrite the symbol as a sum of three parts, a "low-high", a "high-high", and a "high-low" part. Here, high and low refer to the range of frequencies for $q_{j}$, i.e., $k$, compared to $j$ :

$$
\begin{aligned}
q(x, \xi) & =\sum_{j \geq 0}\left(\sum_{k<j-3} q_{k j}(x)+\sum_{k=j-3}^{j+3} q_{k j}(x)+\sum_{k>j+3} q_{k j}(x)\right) \psi_{j}(\xi) \\
& =q_{1}(x, \xi)+q_{2}(x, \xi)+q_{3}(x, \xi) .
\end{aligned}
$$

We will treat each term separately, starting with the simpler piece $q_{2}$.

Since $q_{2}(x, D) f=\sum_{k>0}\left(\sum_{k=j-3}^{j+3} q_{k j} f_{j}\right)$ in $\mathcal{S}^{\prime}$, where $\mathcal{F}\left(\sum_{k=j-3}^{j+3} q_{k j} f_{j}\right)$ is supported on the ball $B\left(0,2^{j+4}\right)$, by Lemma 3.16

$$
\begin{aligned}
\left\|q_{2}(x, D) f\right\|_{N_{p, q, r}^{s}} & \leq C\left\|\left\{2^{j s}\left\|\sum_{k=j-3}^{j+3} q_{k j} f_{j}\right\|_{M_{q}^{p}}\right\}\right\|_{\ell^{r}} \\
& \leq C\left\|\left\{2^{j s} \sum_{k=j-3}^{j+3}\left\|q_{k j}\right\|_{L^{\infty}}\left\|f_{j}\right\|_{M_{q}^{p}}\right\}\right\|_{\ell^{r}} .
\end{aligned}
$$

But (3.30) implies

$$
\sum_{k=j-3}^{j+3}\left\|q_{k j}\right\|_{L^{\infty}} \leq 2^{j \ell(\delta-1)} \sum_{k=-3}^{3} 2^{-k \ell} \leq C,
$$

with $C$ depending only on $\ell$ and $\delta$. So

$$
\left\|q_{2}(x, D) f\right\|_{N_{p, q, r}^{s}} \leq \tilde{C}\left\|\left\{2^{j s}\left\|f_{j}\right\|_{M_{q}^{p}}\right\}\right\|_{\ell^{r}} \leq \tilde{C}\|f\|_{N_{p, q, r}^{s}} .
$$

In the same fashion, since $\mathcal{F}\left(\sum_{k<j-3} q_{k j} f_{j}\right)$ is supported on a shell centered at the frequency $2^{j}$, by Lemma 3.15,

$$
\begin{aligned}
\left\|q_{1}(x, D) f\right\|_{N_{p, q, r}^{s}} & \leq C\left\|\left\{2^{j s}\left\|\sum_{0 \leq k<j-3} q_{k j} f_{j}\right\|_{M_{q}^{p}}\right\}\right\|_{\ell^{r}} \\
& \leq C\left\|\left\{2^{j s}\left\|\sum_{0 \leq k} q_{k j} f_{j}\right\|_{M_{q}^{p}}\right\}\right\|_{\ell^{r}} \\
& \leq C\left\|\left\{2^{j s}\left\|q_{j}\right\|_{L^{\infty}}\left\|f_{j}\right\|_{M_{q}^{p}}\right\}\right\|_{\ell^{r}} \leq \tilde{C}\|f\|_{N_{p, q, r}^{s}} .
\end{aligned}
$$

In the last line, we used the fact that the $q_{j}$ 's are uniformly bounded in $L^{\infty}$.

Finally, let us examine $q_{3}(x, D) f$. Here $\sum_{k>j+3} q_{k j} f_{j}$ is not supported on balls or shells; hence we cannot directly proceed as above. However, note that in $\mathcal{S}^{\prime}$,

$$
\sum_{j \geq 0} \sum_{k>j+3} q_{k j} f_{j}=\sum_{k>3} \sum_{j<k-3} q_{k j} f_{j},
$$

${ }^{9}$ Note that in this case: $\|f\|_{N_{p, q, r}^{s}} \equiv \|\left\{2^{s j}\left\|f_{j}\right\|_{\left.M_{q}^{p}\right\}_{j \geq 0}} \|_{\ell^{r}}\right.$. 
and $\mathcal{F}\left(\sum_{j \leq k-4} q_{k j} f_{j}\right)$ is now supported on a shell around the frequency $|\xi|=2^{k}$. Applying again Lemma 3.15 yields

$$
\begin{aligned}
\left\|q_{3}(x, D) f\right\|_{N_{p, q, r}^{s}} & =\left\|\sum_{k>3} \sum_{j<k-3} q_{k j} f_{j}\right\|_{N_{p, q, r}^{s}} \\
& \leq C\left\|\left\{2^{k s}\left\|\sum_{j<k-3} q_{k j} f_{j}\right\|_{M_{q}^{p}}\right\}\right\|_{\ell^{r}} \\
& \leq C \|\left\{2^{k s} \sum_{j<k-3}\left\|q_{k j}\right\|_{L^{\infty}}\left\|f_{j}\right\|_{\left.M_{q}^{p}\right\}} \|_{\ell^{r}} .\right.
\end{aligned}
$$

Now, use again (3.30) (where we can set $\delta=1$, since $k \geq 0$ ) to write

$$
\begin{aligned}
& 2^{k s} \sum_{j \leq k-4}\left\|q_{k j}\right\|_{L \infty}\left\|f_{j}\right\|_{M_{q}^{p}} \\
& \leq C \sum_{j \leq k-4} 2^{k(s-\ell)} 2^{j \ell}\left\|f_{j}\right\|_{M_{q}^{p}} \\
& =C \sum_{j \leq k-4} 2^{(k-j)(s-\ell)}\left(2^{j s}\left\|f_{j}\right\|_{M_{q}^{p}}\right),
\end{aligned}
$$

with $C$ depending only on $\ell$. But this is the convolution between $\left\{2^{k(s-\ell)}\right\}$, which is in $\ell^{1}$ as long as $s<\ell$, and $\left\{2^{j s}\left\|f_{j}\right\|_{M_{q}^{p}}\right\}$, which is in $\ell^{r}$ by hypothesis. Therefore, by the Young-Hausdorff inequality,

$$
\begin{aligned}
& \left\|\left\{2^{k s} \sum_{j \leq k-4}\left\|q_{k j}\right\|_{L^{\infty}}\left\|f_{j}\right\|_{M_{q}^{p}}\right\}\right\|_{\ell^{r}} \\
& \leq C \|\left\{\sum _ { j \leq k - 4 } 2 ^ { ( k - j ) ( s - \ell ) } \left(2^{j s}\left\|f_{j}\right\|_{\left.\left.M_{q}^{p}\right)\right\}} \|_{\ell^{r}}\right.\right. \\
& \leq C\left\|\left\{2^{k(s-\ell)}\right\}\right\|_{\ell^{1}}\left\|\left\{2^{j s}\left\|f_{j}\right\|_{M_{q}^{p}}\right\}\right\|_{\ell^{r}} \leq \tilde{C}\|f\|_{N_{p, q, r}^{s}} .
\end{aligned}
$$

Combining (3.33), (3.34), and (3.37) produces the desired estimate.

In particular, it ensues that the operator norm of $p(x, D)$ is dominated by an appropriate seminorm of its symbol in the topology of $C_{*}^{\ell} S_{1, \delta}^{m}$.

Because of the good multiplicative properties of regular symbols, if $p(x, \xi) \in S_{1, \delta}^{0}$ with $\delta<1$, then (3.27) holds for every $s \in \mathbb{R}$. This result extends Proposition 3.14 to include the case $q=1$.

Remark 3.19. Since Lemma 3.15 was used to bound $q_{1}(x, D) f$, the restriction $s>0$ is not necessary for this type of symbol, as long as $\ell>0$. Moreover, the only other ingredient in the proof is (3.29b) or an even weaker estimate, namely $\sup _{j}\left\|\sum_{k \leq j} q_{k j}\right\|_{L^{\infty}}<\infty$. Consequently, it is also possible to relax the regularity assumption to $q(x, \xi) \in L^{\infty} S_{1, \delta}^{0}$ (with obvious notation). If $q(x, \xi) \in C_{*}^{0} S_{1, \delta}^{0}$, (3.34) just barely fails, while (3.33) still holds for $s$ positive. This observation can be formalized further and leads us to study the symbol class $\mathcal{B}^{r} S_{1, \delta}^{m}$ introduced by Y. Meyer, which has important applications to non-linear partial differential equations Tay2].

By using symbol smoothing, we can strengthen our result a bit for the case $\delta<1$, namely we can take $s$ negative (but small) in Theorem 3.17. Essentially, symbol smoothing consists of applying to the symbol standard mollifiers in $x$. For details, we refer to Tay1, Vol. 3. 
Corollary 3.20. Under the hypotheses of Theorem [3.17, assume, in particular, that $\delta<1$. Fix $\gamma \in(\delta, 1)$. Then, for $0<\epsilon<(\gamma-\delta) \ell$,

$$
p(x, \xi): N_{p, q, r}^{-\epsilon+m} \rightarrow N_{p, q, r}^{-\epsilon} .
$$

Proof. Again, we can reduce to the case $m=0$. Recall that a symbol $p(x, \xi) \in$ $C_{*}^{\ell} S_{1, \delta}^{0}, \delta<1$, can be decomposed as follows:

$$
p(x, \xi)=p^{\#}(x, \xi)+p^{b}(x, \xi),
$$

with $p^{\#} \in S_{1, \gamma}^{0}$ and $p^{b} \in C_{*}^{\ell} S_{1, \gamma}^{-(\gamma-\delta) \ell}$ for a chosen $\gamma \in(\delta, 1)$. Therefore,

$$
p^{\#}(x, D): N_{p, q, r}^{-\epsilon} \rightarrow N_{p, q, r}^{-\epsilon}, \quad \forall \epsilon,
$$

while

$$
p^{b}(x, D): N_{p, q, r}^{-\epsilon} \rightarrow N_{p, q, r}^{-\epsilon},
$$

as long as $0<\epsilon<(\gamma-\delta) \ell$. In fact, although there is no good calculus for nonregular symbols, $p^{b}(x, D) \circ(I-\Delta)^{(\gamma-\delta) \ell}$ belongs nevertheless to $C_{*}^{\ell} O P S_{1, \gamma}^{0}$.

3.3. Para-differential operators. Para-differential operators are a powerful tool for the study of non-linear differential equations.

As before, we start by outlining the main points of para-differential calculus, which was first introduced by J.-M. Bony $[\mathrm{Bo}$ and developed by Y. Meyer [Me]. A rather complete treatment can be found again in [Tay1, Vol. 3.

Meyer's idea is simple, yet ingenious. It consists of writing a non-linear differential operator as a sum of a linear pseudo-differential operator (which will be of type $(1,1))$ plus a smooth remainder.

Suppose $F: \mathbb{R} \rightarrow \mathbb{R}$ is a smooth (non-linear) function and pick $u$ in some appropriate space. Let $\left\{\phi_{0}, \psi_{j}\right\}, j \geq 1$, be the usual Littlewood-Paley partition of unity. Set $\Psi_{k}=\phi_{0}+\sum_{j \leq k} \psi_{j}$ and write $F(u)$ as

$$
F(u)=F\left(u_{0}\right)+\left[F\left(u_{1}\right)-F\left(u_{0}\right)\right]+\left[F\left(u_{2}\right)-F\left(u_{1}\right)\right]+\cdots
$$

with $u_{k}=\Psi_{k}(D) u$. Applying the Fundamental Theorem of Calculus to each difference gives

$$
\begin{aligned}
F(u) & =\sum_{k=0}^{\infty} m_{k}(u, x) \psi_{k+1}(D) u+F\left(u_{0}\right) \\
& =M_{F}(u, x, D) u+F\left(u_{0}\right),
\end{aligned}
$$

where

$$
m_{k}(u, x)=\int_{0}^{1} F^{\prime}\left(u_{k}+t \psi_{k+1}(D) u\right) d t
$$

and $F\left(u_{0}\right)=R(u)$ is a smooth remainder (recall $\left.\psi_{0}(D) \in O P S^{-\infty}\right) . M_{F}(u, x, D)$ is called a para-differential operator.

Notation 3.21. Even though, in general, $M_{F}$ does depend on $u$ and $F$, it is customary to write $M(x, D)$ instead of $M_{F}(u, x, D)$.

If $u \in L^{\infty}$, then $M_{F}(u, x, D) \in O P S_{1,1}^{0}$, since by the chain rule

$$
\begin{aligned}
\left|D_{\xi}^{\alpha} M(x, \xi)\right| & \leq C_{\alpha}\left\|F^{\prime}\right\|_{L^{\infty}(I)}\langle\xi\rangle^{-|\alpha|}, \\
\left|D_{\xi}^{\alpha} D_{x}^{\beta} M(x, \xi)\right| & \leq C_{\alpha \beta}\left\|F^{\prime \prime}\right\|_{C^{|\beta|-1}(I)}\left\langle\|u\|_{L^{\infty}}\right\rangle^{|\beta|}\langle\xi\rangle^{|\beta|-|\alpha|},
\end{aligned}
$$


where $I$ is an interval containing the essential range of $u$. Note that, in particular, $F$ need not be bounded. But $F$ must be smooth in order to have a regular symbol for $M$. Therefore, if $u \in N_{p, q, r}^{s} \cap L^{\infty}$, results from the previous section imply

$$
M(x, D): N_{p, q, r}^{t} \rightarrow N_{p, q, r}^{t}, \quad \forall t>0 .
$$

Since the operator norm of $p(x, D) \in C_{*}^{\ell} O P S_{1, \delta}^{0}$ depends only on the $C_{*}^{\ell}$-norm of $p(\cdot, \xi)$ from Theorem 3.17, we obtain the bound

$$
\|M(x, D)\|_{\mathcal{L}\left(N_{p, q, r}^{t}\right)} \leq K_{N}(F, u)=C\left\|F^{\prime}\right\|_{C^{N}}\left\langle\|u\|_{L^{\infty}}\right\rangle^{N},
$$

by choosing $N>t$. If $s>0$, we can apply the above estimates to $F(u)$ itself and conclude

$$
\|F(u)\|_{N_{p, q, r}^{s}} \leq K_{N}(F, u)\|u\|_{N_{p, q, r}^{s}}+\|R(u)\|_{N_{p, q, r}^{s}}, \quad N>s .
$$

Actually, so far we know $R(u)$ is smooth, but there is no prescribed behavior at infinity. Since $u$ is bounded, the chain rule again implies $R(u)=F\left(u_{0}\right) \in C^{\ell}$ for every $\ell \geq 0$, i.e.,

$$
\left\|\Delta_{j} F\left(u_{0}\right)\right\|_{L^{\infty}} \leq C_{F} 2^{-j \ell}, \quad \forall \ell \geq 0 .
$$

Since $L^{\infty} \subset M_{q}^{p}, R(u) \in N_{p, q, \infty}^{\ell}$ for all $\ell>0$, and $N_{p, q, \infty}^{\ell} \subset N_{p, q, r}^{s}$ if $\ell>s$.

The following Moser-type estimate can be obtained by specializing (3.46) to $F(u)=u^{2}$,

$$
\left\|u^{2}\right\|_{N_{p, q, r}^{s}} \leq C_{s}\|u\|_{L^{\infty}}\|u\|_{N_{p, q, r}^{s}}, \quad \forall u \in L^{\infty} \cap N_{p, q, r}^{s}, s>0 .
$$

Indeed, in this case $\left\|F^{\prime}\right\|_{L^{\infty}}+\left\|F^{\prime \prime}\right\|_{L^{\infty}}\left\langle\|u\|_{L^{\infty}}\right\rangle \approx 2\left\langle\|u\|_{L^{\infty}}\right\rangle$, and no other term arises because higher-order derivatives of $F$ are zero.

Let us now consider a function of several variables $F(u)=F\left(u^{1}, \ldots, u^{m}\right)$. Then, expanding $F$ as before gives

$$
F(u)=\sum_{i=0}^{m} M_{F, i}(u, x, D) u+F\left(\psi_{0}(D) u\right),
$$

where

$$
M_{F, i}(x, \xi)=\sum_{k=0}^{\infty} m_{k}^{i}(x) \psi_{k+1}(\xi)
$$

and

$$
m_{k}^{i}(x)=\int_{0}^{1} \partial_{i} F\left(\Psi_{k}(D) u+t \psi_{k+1}(D) u\right) d t .
$$

Hence, as long as each $u^{i}$ belongs to $L^{\infty}$, all the results of the one-dimensional case extend. In particular, for $F(u, v)=u \cdot v$, (3.49) becomes

$$
u \cdot v=\tilde{M}_{F}(u, x, D) v+\tilde{M}_{F}(v, x, D) u+\psi_{0}(D) u \psi_{0}(D) v,
$$

with

$$
\tilde{M}_{F}(u, x, \xi)=\sum_{k \geq 0}\left[\Psi_{k}(D) u+\frac{1}{2} \psi_{k+1}(D) u\right] \psi_{k+1}(\xi) .
$$

The equivalent of (3.44) is

$$
\begin{aligned}
\left\|\tilde{M}_{F}(u, \cdot, \xi)\right\|_{C^{\ell}} & \leq C \sum_{k \geq 0} 2^{k \ell}\|u\|_{L^{\infty}} \psi_{k+1}(\xi) \\
& \approx C\|u\|_{L^{\infty}}\langle\xi\rangle^{\ell}
\end{aligned}
$$


since $\langle\xi\rangle^{\ell} \sim 2^{k \ell}$ on Supp $\psi_{k+1}$. Therefore, a Moser-type estimate follows:

$$
\|u \cdot v\|_{N_{p, q, r}^{s}} \leq C\left(\|u\|_{L^{\infty}}\|v\|_{N_{p, q, r}^{s}}+\|u\|_{N_{p, q, r}^{s}}\|v\|_{L^{\infty}}\right) .
$$

It is interesting, especially in view of applications to the Navier-Stokes equation 10 to establish when the space $N_{p, q, r}^{s}$ is an algebra. Given the discussion above, it is enough to find when $N_{p, q, r}^{s} \hookrightarrow L^{\infty}$.

Recall first the situation for Besov spaces [Ru1]. For $0<p_{0} \leq p_{1} \leq \infty, 0<q \leq$ $\infty$ and $-\infty \leq s_{1} \leq s_{0}<\infty$,

$$
B_{p_{0}, q}^{s_{0}} \hookrightarrow B_{p_{1}, q}^{s_{1}} \quad \text { if } s_{0}-\frac{n}{p_{0}}=s_{1}-\frac{n}{p_{1}} .
$$

But $B_{\infty, \infty}^{s}=C_{*}^{s}$, so that

$$
B_{p, q}^{s+n / p} \hookrightarrow L^{\infty} \quad \text { if } s>0,0<p, q \leq \infty .
$$

Also, in the critical case $s=n / p, B_{p, q}^{n / p} \subset L^{\infty}$ for $0<q \leq 1$. Both these embeddings are sharp [SiT].

Combining (3.55) and (3.56) with Theorem 2.4 yields immediately

$$
N_{p, q, r}^{s} \hookrightarrow L^{\infty} \text { for } s>n / p, 1<r \leq \infty,
$$

and

$$
N_{p, q, 1}^{s} \hookrightarrow L^{\infty} \quad \text { for } s \geq n / p .
$$

Recall also the relation with BMO established in Sections 2.1 and 2.2.

Accordingly, (3.54) provides the following criterion.

Corollary 3.22. The space $N_{p, q, r}^{s}$ is a Banach algebra for

(1) $s>n / p, 1 \leq q \leq p<\infty$, if $r \in(1, \infty]$, or

(2) $s \geq n / p, 1 \leq q \leq p<\infty$, if $r=1$.

Another way of decomposing a product of two functions is the paraproduct of J.-M. Bony [Bo]. We follow [Tay2] and define it as

$$
\pi(v, w)=\sum_{k \geq 1}\left(\Psi_{k-1}(D) v\right)\left(\psi_{k+1} w\right) .
$$

So, $\pi(u, v)$ is obtained by applying symbol smoothing directly to the multiplication operator by $v, m_{v}$. When $v \in C^{\ell}, \ell>0$, one has

$$
v \cdot w=\pi(v, w)+\rho_{v}(x, D) w,
$$

with $\rho_{v}(x, \xi) \in C^{\ell} S_{1,1}^{-\ell}$. Note that, by the results of the previous section,

$$
\rho_{v}(x, D): N_{p, q, r}^{s-\ell} \rightarrow N_{p, q, r}^{s}
$$

only if $0<s<\ell$.

To compare the paraproduct with (3.43) $)$, set $v=F^{\prime}(u)$. Then

$$
M_{F}(x, \xi)-\pi_{F^{\prime}}(x, \xi) \in S_{1,1}^{-\ell},
$$

if also $u \in C^{\ell}, \ell>0$ [Tay2, which allows us to improve slightly upon (3.60).

\footnotetext{
${ }^{10}$ Recall the non-linearity in the Navier-Stokes equation is quadratic.
} 
Corollary 3.23. Let $F$ be a smooth function. If $u \in C^{\ell} \cap N_{p, q, r}^{s}$, with $s>0, \ell>0$, the following decomposition holds:

$$
F(u)=\pi\left(F^{\prime}(u), u\right)+\tilde{R}(u),
$$

where $\tilde{R}(u) \in N_{p, q, r}^{s+\ell}$.

In particular, the best approximation to $u^{2}$ is not $\pi(u, u)$, but rather $2 \pi(u, u)$.

Proof. Write

$$
F(u)=\pi\left(F^{\prime}(u), u\right)+\left(M_{F}(x, D) u-\pi\left(F^{\prime}(u), u\right)\right)+R(u),
$$

with $\tilde{R}(u)=\left(M_{F}(x, D) u-\pi\left(F^{\prime}(u), u\right)\right)+R(u)$. But $R(u)$ is smooth and $\left(M_{F}(x, D) u\right.$ $\left.-\pi\left(F^{\prime}(u), u\right)\right)=Q_{F}(x, D) u$ belongs to $N_{p, q, r}^{s+\ell}$ because of (3.61).

The remainder term $\rho_{u}$ in (3.60) can be decomposed further to obtain a form of paraproduct that matches closely the expansion (3.31) for an elementary symbol and emphasizes the symmetry between $u$ and $v$. Set

$$
u \cdot v=T_{u} v+T_{v} u+R(u, v),
$$

where

$$
\begin{aligned}
& T_{u} v=\sum_{j \geq 1} \Psi_{j-2}(D) u \psi_{j}(D) v, \\
& R(u, v)=R_{u} v=\sum_{|k-j| \leq 1} \psi_{k}(D) u \psi_{j}(D) v .
\end{aligned}
$$

Note that both $T_{u}$ and $R_{u}$ have symbols of the form discussed in Remark 3.19 Precisely, $T_{u}$ is a symbol of "type" $q_{1}$, while $R_{u}$ is of "type" $q_{2}$.

Here, $q_{k j}=\psi_{k}(D) u$, if $k \leq j-2$ or $j-1 \leq k \leq j+1$ respectively, and zero otherwise (in the notation of Section (3.2). Therefore, whenever the $q_{k j}$ 's are uniformly bounded in $L^{\infty}$, that is $u \in C_{*}^{0}$,

$$
R_{u}: N_{p, q, r}^{s} \rightarrow N_{p, q, r}^{s}, \quad T_{u}: N_{p, q, r}^{s} \rightarrow N_{p, q, r}^{s-\epsilon},
$$

for all $\epsilon>0$, as long as $s>0$. In fact, a similar analysis shows that, if $u \in C_{*}^{-\mu}$, $\mu>0, T_{u}$ and $R_{u}$ belong to the operator class $O P S_{1,1}^{\mu}$, so that

$$
\begin{array}{ll}
\left\|T_{u} v\right\|_{N_{p, q, r}^{s-\mu}} \leq C\|u\|_{C_{*}^{-\mu}}\|v\|_{N_{p, q, r}^{s}} & \forall \mu>0, \\
\left\|R_{u} v\right\|_{N_{p, q, r}^{s-\mu}} \leq C\|u\|_{C_{*}^{-\mu}}\|v\|_{N_{p, q, r}^{s}} & \forall \mu \in \mathbb{R}, s>\mu .
\end{array}
$$

We can then extend the Moser estimates (3.54):

$$
\begin{aligned}
& \|u \cdot v\|_{N_{p, q, r}^{s-\mu}} \leq C\left(\|u\|_{C_{*}^{-\mu}}\|v\|_{N_{p, q, r}^{s}}+\|u\|_{N_{p, q, r}^{s}}\|v\|_{C_{*}^{-\mu}}\right), \quad \forall \mu>0, s>\mu \\
& \|u \cdot v\|_{N_{p, q, r}^{s+-n / p}} \leq C\left(\|u\|_{N_{p, q, r}^{s}}\|v\|_{N_{p, q, r}^{\ell}}^{\ell}\right), \quad \text { if } s+\ell>n / p
\end{aligned}
$$

For $s=n / p$, in particular,

$$
\|u \cdot v\|_{N_{p, q, r}^{n / p-\epsilon}} \leq C\|u\|_{N_{p, q, r}^{n / p}}\|v\|_{N_{p, q, r}^{n / p}}, \quad \forall \epsilon>0,
$$

which shows how close $N_{p, q, r}^{n / p}$ is to a Banach algebra. 


\section{Extension to MANifolds}

The aim of this section is to define the BM spaces on compact manifolds. In hydrodynamics, boundary value problems are a rather natural setting. At the same time, due to the incompressibility constraint, non-local effects are likely to become relevant. Even when boundaries are not present, curvature terms may not be negligible.

We will concentrate on the simplest case, that of a (smooth) compact manifold $M$ without boundary. We plan to address the question of manifolds with boundary in future work.

It will be necessary to use pseudo-differential calculus both on BM and Morrey spaces. Consequently, we are able to consider only the inhomogeneous spaces $N_{p, q, r}^{s}$, $q>1$.

4.1. Main definition. A natural way to extend the definition of a function space $X$ from $\mathbb{R}^{n}$ to compact manifolds is by partitions of unity and coordinate charts. Then any property that is localizable also extends. There are clearly two steps to this process:

(1) given $f \in X\left(\mathbb{R}^{n}\right)$ and $\phi \in C_{0}^{\infty}\left(\mathbb{R}^{n}\right)$, show $\phi \cdot f \in X\left(\mathbb{R}^{n}\right)$;

(2) given a diffeomorphism $\chi$ of $\mathbb{R}^{n}$, show that the topology of $X\left(\mathbb{R}^{n}\right)$ is invariant under the action of $\chi$.

Here, as throughout the section, we implicitly identify a coordinate chart $U$ on $M$ with its image in $\mathbb{R}^{n}$ and, consequently, $\chi$ represents a certain coordinate transformation. By refining the charts on $M$, if necessary, one can reduce to considering a diffeomorphism that is linear outside a compact set $K$. In particular, the Jacobian of the transformation $|\partial \chi / \partial x|$ is bounded by some constant $A>0$.

Notation 4.1. Since we are primarily concerned with the inhomogeneous space $N_{p, q, r}^{s}$, here $\left\{\psi_{j}\right\}_{j \geq 0}$ is a Littlewood-Paley partition of unity as in (3.15)), i.e., $\psi_{j}$ is supported on the dyadic shell $D_{j}=\left\{\xi \in \mathbb{R}^{n}\left|2^{j-2} \leq\right| \xi \mid \leq 2^{j}\right\}$ for $j>0$, but $\psi_{0}$ is supported on the ball $B(0,2)$.

Recall first how the Morrey spaces $M_{q}^{p}, 1 \leq q \leq p<\infty$, are defined on manifolds. It is enough to prove that $M_{q}^{p}$ is invariant under the action of $\chi$, since it is immediate that the multiplication operator $m_{\phi}$, where $m_{\phi} f=\phi f$, is bounded on $M_{q}^{p}$. An easy change of variable gives

$$
\left\|\chi^{*} \circ f\right\|_{M_{q}^{p}} \leq A^{1 / q} \sup _{\substack{0<R \leq 1 \\ x_{0} \in \mathbb{R}^{n}}}\left[R^{n / p-n / q}\left(\int_{\chi\left(B\left(x_{0}, R\right)\right)}|f(y)|^{q} d y\right)^{1 / q}\right],
$$

where $\chi^{*}$ is the pull-back on functions, i.e., $\chi^{*} f(x)=f(\chi(x))$. Even though $\chi\left(B\left(x_{0}, R\right)\right)$ is not a ball in general, $\chi\left(B\left(x_{0}, R\right)\right)$ is covered by a finite number $N$ of balls of radius $R$, where $N$ is independent of $x \in \mathbb{R}^{n}$ and $R \in(0,1]$, since $\chi$ is linear outside $K$ compact. Hence,

$$
\left\|\chi^{*} \circ f\right\|_{M_{q}^{p}} \leq N A^{1 / q} \sup _{\substack{0<R \leq 1 \\ x_{0} \in \mathbb{R}^{n}}}\left[R^{n / p-n / q}\left(\int_{B\left(x_{0}, R\right)}|f(y)|^{q} d y\right)^{1 / q}\right] \leq C\|f\|_{M_{q}^{p}} .
$$

Definition 4.2. The space $M_{q}^{p}(M)$ is the set of all $f \in \mathcal{D}^{\prime}(M)$ such that, for every coordinate patch $U$ on $M$ and $\phi \in C_{0}^{\infty}(U), \phi f \in M_{q}^{p}\left(\mathbb{R}^{n}\right)$. 
Next, we proceed similarly in identifying $N_{p, q, r}^{s}(M)$. However, the situation is not as straightforward, because the Littlewood-Paley theory does not carry over directly to manifolds. Indeed, each $\psi_{j}(D)$ is inherently a non-local operator. Moreover, only the principal symbol of a pseudo-differential operator is a well-defined covariant object. We are forced to deal with asymptotic expansions and equivalence classes of symbols.

Remark 4.3. Note that, obviously, $M_{q}^{p}(M) \approx \mathcal{M}_{q}^{p}(M)$, if $M$ is compact. On the other hand, compactly-supported elements of $N_{p, q, r}^{s}\left(\mathbb{R}^{n}\right)$ and $\mathcal{N}_{p, q, r}^{s}\left(\mathbb{R}^{n}\right)$ do not necessarily coincide. Take, for example, $f=\chi_{B(0,1)}$, the characteristic function of the unit ball in $\mathbb{R}^{n}$. We can estimate its Morrey norm as follows. First,

$$
\psi_{j}(D) f(x)=\int_{B(x, 1)} 2^{j n} \check{\psi}\left(2^{j} y\right) d y=\int_{B\left(x, 2^{j}\right)} \check{\psi}(z) d z
$$

where $\psi=\psi_{1}$. Since $\check{\psi}$ is rapidly decreasing at infinity, the largest contribution to $\left\|\psi_{j}(D) f\right\|_{M_{q}^{p}}$ comes from picking $x=0$, so that

$$
\left\|\psi_{j}(D) f\right\|_{M_{q}^{p}} \approx C\left|\int_{B\left(0,2^{j}\right)} \check{\psi}(z) d z\right| .
$$

Choose $s$ negative. Then

$$
\begin{aligned}
\|f\|_{N_{p, q, r}^{s}} & =\left[\sum_{j>0}\left(2^{j s}\left\|\psi_{j}(D) f\right\|_{\left.M_{q}^{p}\right)^{r}}\right]^{1 / r}+\left\|\psi_{0}(D) f\right\|_{M_{q}^{p}}\right. \\
& \leq C\left\|\check{\psi}_{0}\right\|_{L^{1}}\left(\sum_{j>0} 2^{-j|s|}\right)+\left\|\psi_{0}(D) f\right\|_{M_{q}^{p}}<\infty .
\end{aligned}
$$

On the other hand, if $|s|>n$ the low-frequency part $(j<0)$ determines a divergent contribution to the norm of $\mathcal{N}_{p, q, r}^{s}$. As a matter of fact, since $\check{\psi}(0)=\int_{\mathbb{R}^{n}} \psi(\xi) d \xi>$ $0, \check{\psi}$ is strictly positive on some neighborhood of the origin. (4.3) then gives

$$
\left\|\psi_{j}(D) f\right\|_{M_{q}^{p}} \geq C 2^{-|j| n},
$$

for $j$ negative large enough (say $j<j_{0}$ ), where the constant $C$ depends on $n$ and $\check{\psi}$, but not on $j$. Consequently,

$$
\|f\|_{\mathcal{N}_{p, q, r}^{s}} \geq\left[\sum_{j<j_{0}}\left(2^{j s}\left\|\psi_{j}(D) f\right\|_{M_{q}^{p}}\right)^{r}\right]^{1 / r} \geq C\left[\sum_{j<j_{0}}\left(2^{|j|(|s|-n)}\right)^{r}\right]^{1 / r} .
$$

But the last sum diverges, given the choice of $s$.

So, in principle, $\mathcal{N}_{p, q, r}^{s}(M) \not \approx N_{p, q, r}^{s}(M)$. One could try to use interpolation (cf. Proposition 2.6) to define the homogeneous spaces, but this is not a good working definition, as we have seen already in Section 3.1

Let $U$ be a coordinate patch on $M$ and $\phi \in C^{\infty}(M)$ be supported on $U$. The multiplication operator $m_{\phi}$ is a pseudo-differential operator in $O P S_{1,0}^{0}$. Therefore, it is bounded on $N_{p, q, r}^{s}$ by virtue of Proposition [3.14, and we can restrict to functions $f$ supported on coordinate patches. 
The next necessary ingredient is to prove that the action of a diffeomorphism preserves the topology of $N_{p, q, r}^{s}\left(\mathbb{R}^{n}\right)$. We have to estimate

$$
\left\|\left\{2^{j s}\left\|\psi_{j}(D)\left(\chi^{*} f\right)\right\|_{M_{q}^{p}\left(\mathbb{R}^{n}\right)}\right\}\right\|_{\ell^{r}} .
$$

But, since $\chi^{*}$ maps $M_{q}^{p}\left(\mathbb{R}^{n}\right)$ to itself, it is equivalent to showing instead that

$$
\left\|\left\{2^{j s}\left\|\chi^{*-1} \psi_{j}(D) \chi^{*} f\right\|_{M_{q}^{p}\left(\mathbb{R}^{n}\right)}\right\}\right\|_{\ell^{r}} \leq C(\chi, s, p, q, r)\|f\|_{N_{p, q, r}^{s}} .
$$

The advantage is that the action by conjugation of a diffeomorphism on a pseudodifferential operator (at least of type $(1, \delta)$ with $\delta<1 / 2$ ) can be treated using Egorov's theorem, and there is a complete asymptotic expansion of the resulting symbol (see [Tay4 or [Tr] for a proof).

For convenience, we denote the symbol of $\chi^{*-1} \psi_{j}(D) \chi^{*}$ with $p_{j}(x, \xi)$. The following expansion holds:

$$
\left.p_{j}(x, \xi) \sim \sum_{\alpha} \frac{1}{\alpha !} \partial_{\xi}^{\alpha} D_{z}^{\alpha} q_{j}(x, \xi, z)\right|_{z=x} \sim \sum_{k} r_{j}^{(k)}(x, \xi),
$$

where

$$
q_{j}(x, \xi, z)=\psi_{j}\left(H(x, z)^{t-1} \xi\right)\left|\frac{\partial \chi}{\partial z}\right| \frac{1}{|H(x, z)|} \phi(x, z),
$$

with $H(x, z)$ defined by

$$
\chi(x)-\chi(z)=H(x, z)(x-z),
$$

and $r_{j}^{(k)}(x, \xi)$ is obtained by collecting all the terms in the expansion of the same order, namely $|\alpha|=k$. Since clearly $H(x, x)=\partial \chi / \partial x$, which is non-zero by hypothesis, $H(x, z)$ is well-defined and smooth near the diagonal in $\mathbb{R}^{n} \times \mathbb{R}^{n}$. The function $\phi$ is supported in a neighborhood of $x=z$ and acts as a regularizing factor, as $H$ may not be defined everywhere. The error introduced in this way is a symbol supported in $(x, z)$ away from the diagonal; thus it is of order $-\infty$ and does not contribute to the asymptotic expansion.

Note that, even though each term in (4.9) is the symbol of a smoothing operator, its norm depends on $j$, while we want uniform estimates. Since $\psi_{j}$ is supported on the shell $D_{j}$ where $\langle\xi\rangle \sim 2^{j}$, it follows that $\left\{r_{j}^{(k)}(x, \xi)\right\}_{j \geq 0}$ is bounded in $S_{1,0}^{-k}$ and $\left\{2^{j l} r_{j}^{(k)}(x, \xi)\right\}_{j \geq 0}$ is $O\left(2^{l-k}\right)$ in $S_{1,0}^{0}$ for $0 \leq l \leq k$.

Let us concentrate first on the principal symbol of $p_{j}(x, \xi)$. Write

$$
p_{j}(x, \xi)=\psi_{j}\left(\frac{\partial \chi}{\partial x} \stackrel{t-1}{(x)} \xi\right)+p_{j}^{(1)}(x, \xi)=r_{j}^{(0)}(x, \xi)+p_{j}^{(1)}(x, \xi) .
$$

Again, the $\xi$-support of $\psi_{j}\left(\frac{\partial \chi}{\partial x} \stackrel{t-1}{(x)} \xi\right)$ can be covered by a finite number $N$, independent of $x$, of shells $D_{m}$, as the Jacobian matrix $\frac{\partial \chi}{\partial x}$ is constant outside a compact set. Therefore,

$$
\psi_{j}\left(\frac{\partial \chi}{\partial x} \stackrel{t-1}{(x) \xi)}=\sum_{m=j-N}^{j+N} \psi_{j}\left(\frac{\partial \chi}{\partial x} \stackrel{t-1}{(x) \xi)} \psi_{m}(\xi) .\right.\right.
$$


But $\left\{\psi_{j}\left(\frac{\partial \chi}{\partial x} \stackrel{t-1}{(x)} \xi\right)\right\}_{j \geq 0}$ is bounded in $S_{1,0}^{0}$. Accordingly, these symbols define operators $Q_{j}$ that are uniformly bounded in $N_{p, q, r}^{s}$ and

$$
\left\|Q_{j} f\right\|_{M_{q}^{p}} \leq \sum_{k=j-N}^{j+N}\left\|Q_{j} \circ \psi_{k}(D) f\right\|_{M_{q}^{p}} \leq C \sum_{k=j-N}^{j+N}\left\|\psi_{k}(D) f\right\|_{M_{q}^{p}}
$$

with $C$ independent of $j$. Furthermore,

$$
\left\|\left\{2^{j s}\left\|Q_{j} f\right\|_{M_{q}^{p}}\right\}\right\|_{\ell^{r}} \leq C\left\|\left\{\sum_{k=j-N}^{j+N} 2^{(j-k) s} 2^{k s}\left\|\psi_{k}(D) f\right\|_{M_{q}^{p}}\right\}\right\|_{\ell^{r}} \leq \tilde{C}\|f\|_{N_{p, q, r}^{s}},
$$

by the Young-Hausdorff inequality (cf. proof of Theorem[3.17).

Next, we look at the residual term $p_{j}^{(1)}$. From (4.9),

$$
\begin{aligned}
p_{j}^{(1)}(x, \xi) & =\left.\sum_{i=1}^{n} \partial_{\xi_{i}} D_{z_{i}} q_{j}(x, \xi, z)\right|_{z=x}+p_{j}^{(2)}(x, \xi) \\
& =r_{j}^{(1)}(x, \xi)+p_{j}^{(2)}(x, \xi)
\end{aligned}
$$

with $2^{2 j} p_{j}^{(2)}(x, \xi)$ bounded uniformly in $j$ in $S_{1,0}^{0} \cdot r_{j}^{(1)}$ can be written as a finite sum of terms of the form

$$
h_{j}(x) g_{j}(x, \xi),
$$

with $h_{j}(x)$ uniformly bounded in $L^{\infty}$ and $g_{j}$ smooth with compact support in both $x$ and $\xi$, uniformly bounded in $S_{1,0}^{0}$. Moreover, since $g_{j}$ is basically either $2^{-j} \psi_{j}^{\prime \prime}\left(H(x, z)^{t-1} \xi\right)\left(2^{j} \xi_{i}\right)(i=1, \cdots, n)$ or $2^{-j} \psi_{j}^{\prime}\left(H(x, z)^{t-1} \xi\right)$, estimates similar to (4.13) apply. Hence, an analysis parallel to that of $r_{j}^{(0)}$ can be carried out yielding

$$
\left\|\left\{2^{j s}\left\|r_{j}^{(1)}(\cdot, D) f\right\|_{M_{q}^{p}}\right\}\right\|_{\ell^{r}} \leq C\|f\|_{N_{p, q, r}^{s}} .
$$

Remark 4.4. Note that, when differentiating $q_{j}(x, \xi, z)$ with respect to $z$, factors of $\xi_{i}$ are produced. However, they do not change estimates of the symbol for large $j$, since they always appear in the combination $2^{-j} \xi_{i}$, which is $O(1)$.

Iterating this process takes care of any finite number of terms in the expansion (4.9). At the $k$-th step,

$$
p_{j}(x, \xi)=\sum_{l \leq k-1} r_{j}^{(l)}(x, \xi)+p_{j}^{(k)}(x, \xi),
$$

where all the $r_{j}^{(l)}(x, \xi)$ share a form similar to (4.17) and satisfy an estimate similar to (4.18), while the remainder is such that $\left\{2^{j l} p_{j}^{(k)}(x, \xi)\right\}_{j \geq 0}$ is bounded in $S_{1,0}^{l-k}$ if $0 \leq l \leq k$. So, if $k$ is large enough $(k>\max (s+1, n / p+2)$ for $s \geq 0$ and $k>n / p+2-s$ if $s<0)$ and $l \leq \max (s, 0)+1$, we have the following estimate:

$$
\begin{aligned}
2^{j l}\left\|p_{j}^{(k)}(x, D) f\right\|_{M_{q}^{p}} & \leq 2^{j l}\left\|p_{j}^{(k)}(x, D) f\right\|_{L^{\infty}} \leq 2^{j l}\left\|p_{j}^{(k)}(x, D) f\right\|_{N_{p, q, r}^{n / p+1}} \\
& \leq C_{l, p, q, r}\|f\|_{N_{p, q, r}^{n / p+1-(k-l)}} \leq C_{l, p, q, r}\|f\|_{N_{p, q, r}^{s}}
\end{aligned}
$$


by Sobolev embedding. (Recall $L^{\infty} \subset M_{q}^{p}$.) Then, choose $l=\max (s, 0)+\epsilon, 0<$ $\epsilon<1$ to obtain

$$
\begin{aligned}
\left\|\left\{2^{j s}\left\|p_{j}^{(k)}(x, D) f\right\|_{M_{q}^{p}}\right\}\right\|_{\ell^{r}} & \leq\left\|\left\{2^{j(l-\epsilon)}\left\|p_{j}^{(k)}(x, D) f\right\|_{M_{q}^{p}}\right\}\right\|_{\ell^{r}} \\
& \leq\left\|\left\{2^{-\epsilon j}\right\}\right\|_{\ell^{\infty}}\left\|\left\{2^{j l}\left\|p_{j}^{(k)}(x, D) f\right\|_{M_{q}^{p}}\right\}\right\|_{\ell^{r}} \\
& \leq C_{s, p, q, r}\|f\|_{N_{p, q, r}^{s}} .
\end{aligned}
$$

(4.8) now follows from (4.15), (4.18), and (4.21).

Definition 4.5. A distribution $f$ on $M$ belongs to $N_{p, q, r}^{s}(M)$ if, for every coordinate patch $U$ on $M$ and $\phi \in C_{0}^{\infty}(U), \phi f \in N_{p, q, r}^{s}\left(\mathbb{R}^{n}\right)$. Then, we set for each $f \in$ $N_{p, q, r}^{s}(M)$ :

$$
\|f\|_{N_{p, q, r}^{s}(M)}=\sup _{\left\{\phi_{\alpha}\right\}}\left\{\sum_{\alpha}\left\|\phi_{\alpha} f\right\|_{N_{p, q, r}^{s}\left(\mathbb{R}^{n}\right)}\right\}
$$

where the supremum is taken over all possible representations of $f$ in terms of partitions of unity $\left\{\phi_{\alpha}\right\}$ supported on coordinate patches $U_{\alpha}$.

4.2. Equivalent norms. When considering spaces on manifolds, it is useful to work in a covariant setting. Clearly, the operator $D=(1 / i) \partial$ cannot be used. A covariantly defined operator that is intimately related to the geometry of a manifold is the Laplace operator $\Delta$. It is, therefore, natural to try to define the topology of $N_{p, q, r}^{s}(M)$ in terms of functions of $\sqrt{-\Delta}$, an operator of order one such as $D$.

As before, we assume that $M$ is smooth, compact, without boundary. We assume further that $M$ is Riemannian with metric $g=\left[g_{i j}\right]$. We shall write $\Delta_{g}$ for the Laplace operator associated to the metric $g$, i.e.,

$$
\Delta_{g}=(\operatorname{det} g)^{-1 / 2} \partial_{j} g^{j k}(\operatorname{det} g)^{1 / 2} \partial_{k},
$$

while $\Delta$ still denotes the Euclidean Laplacian, and we will identify $\Delta_{g}$ with its local representation on $\mathbb{R}^{n}$ via a coordinate system.

Families of operators of the form $\rho\left(\delta \sqrt{-\Delta_{g}}\right)$, where $\rho$ is an even, rapidly decreasing function, and $\delta \in(0,1]$, are called approximate identities if $\rho(\xi)=1$ in a neighborhood of the origin. They have several applications to the theory of partial differential equations on manifolds (see [ST], for example).

Remark 4.6. Let $\left\{\psi_{j}\right\}$ be the Littlewood-Paley partition of unity used in Section 4.1 Recall that, by construction, $\psi_{j}=\psi\left(2^{-j} \xi\right)$ for $j>0$, where $\psi \equiv \psi_{1}$. In particular, there is no loss of generality in assuming that $\psi$ is radial, i.e., $\psi(\xi)=\tilde{\psi}(|\xi|)$ for some function $\tilde{\psi}$ on $\mathbb{R}$. With abuse of notation, we identify $\psi$ with $\tilde{\psi}$. Then, $\psi$ corresponds to $\rho$ above and $2^{-j}$ to $\delta$.

The main result of this section is the following theorem.

Theorem 4.7. Let $s \in \mathbb{R}, 1<q \leq p<\infty, r \in[1, \infty]$. Let $\left\{\psi_{j}\right\}, j \geq 0$, be a Littlewood-Paley partition of unity. Then, an equivalent norm in $N_{p, q, r}^{s}(M)$ is given by

$$
\|f\|_{N_{p, q, r}^{s}(M)}^{*}=\left\|\left\{2^{j s}\left\|\psi_{j}\left(\sqrt{-\Delta_{g}}\right) f\right\|_{M_{q}^{p}(M)}\right\}\right\|_{\ell^{r}} .
$$

Intuitively, (4.24) is true, because the principal symbol of $\psi_{j}\left(\sqrt{-\Delta_{g}}\right)$ still behaves like a bump function with a "shell-like" support, even though this support is now deformed by the non-Euclidean metric. 
We follow Schrader and Taylor [ST] in part, but our derivation is admittedly very similar to that of Seeger and Sogge [SeSo] for Besov and Triebel-Lizorkin spaces. However, their argument is simplified owing to the nice properties of $L^{p}$ spaces. In particular, they can reduce to considering a $C^{\infty}$ function and do not have to bound remainder terms. On the other hand, smooth functions are not dense in BM spaces.

The core of the proof again consists of deriving careful pseudo-differential estimates. Unfortunately, operators on manifolds correspond to equivalence classes of symbols, as already mentioned. Hence, we must deal with infinite expansions at each step.

We use the following asymptotic expansion derived in [ST:

$$
\sigma\left(\rho\left(\delta \sqrt{-\Delta_{g}}\right)\right) \sim \sum_{k \geq 0} \delta^{2 k} \rho^{(2 k)}\left(\delta|\xi|_{x}\right) p_{k}(x, \delta \xi) .
$$

Here, $p_{k}(x, \delta \xi)$ is a classical symbol of order $k, \rho^{(2 k)}$ is the $2 k$-th derivative of $\rho$ with respect to its argument, and $|\xi|_{x}=\left(\sum_{i, j} g^{i j}(x) \xi_{i} \xi_{j}\right)^{1 / 2}$ is the norm of the covector $(x, \xi)$ in the cotangent bundle $T^{*}(M)$.

The expansion above is obtained by formally writing $\rho(\delta \sqrt{-\Delta})$ in terms of its cosine transform ( $\rho$ is an even function)

$$
\rho\left(\delta \sqrt{-\Delta_{g}}\right) u(x)=\frac{1}{2 \pi^{1 / 2}} \int_{-\infty}^{\infty} \hat{\rho_{\delta}}(t) \cos \left(t \sqrt{-\Delta_{g}}\right) u(x) d t,
$$

where $\rho_{\delta}(\tau)=\rho(\delta \tau)$. Recall that $\cos \left(t \sqrt{-\Delta_{g}}\right)$ is (half of) the propagator for the wave equation and, consequently, its kernel satisfies the finite propagation speed property. It follows that the Schwartz kernel of $\rho\left(\delta \sqrt{-\Delta_{g}}\right)$ is rapidly decreasing, as $\delta \rightarrow 0$, outside an arbitrarily small region around the diagonal in $M \times M$. Therefore, one can limit integration in (4.26) to $|t| \leq T_{0}, T_{0}$ small, by localizing $\hat{\rho_{\delta}}$ to a neighborhood of the origin, the error being a smoothing operator that is rapidly decreasing in $\delta$ and, thus, negligible. Moreover, one can work in local coordinates.

In this situation, it is convenient to use geometrical optics methods and stationary phase approximation, which leads to (4.25). In particular, the appearance of only even-order derivatives is due to an extra symmetry that the phase functions and amplitudes possess.

To proceed with the proof of the theorem, we first restrict to a coordinate patch $U$, which amounts to proving

$$
\left\|\left\{2^{j s}\left\|\psi_{j}\left(\sqrt{-\Delta_{g}}\right)(\phi f)\right\|_{M_{q}^{p}(M)}\right\}\right\|_{\ell^{r}} \leq C\left\|\left\{2^{j s}\left\|\psi_{j}\left(\sqrt{-\Delta_{g}}\right) f\right\|_{M_{q}^{p}(M)}\right\}\right\|_{\ell^{r}}
$$

for all $\phi \in C_{0}^{\infty}(U)$. Since the multiplication operator $m_{\phi} \in O P S_{1,0}^{0}$, clearly,

$$
\left\|\left\{2^{j s}\left\|\phi\left(\psi_{j}\left(\sqrt{-\Delta_{g}}\right) f\right)\right\|_{M_{q}^{p}(M)}\right\}\right\|_{\ell^{r}} \leq C\left\|\left\{2^{j s}\left\|\psi_{j}\left(\sqrt{-\Delta_{g}}\right) f\right\|_{M_{q}^{p}(M)}\right\}\right\|_{\ell^{r}} .
$$

Then we switch the order of the product and attempt to bound the commutator $\left[\psi_{j}\left(\sqrt{-\Delta_{g}}\right), m_{\phi}\right]$ appropriately. Alternatively, notice that $m_{\phi}$ and $\psi_{j}\left(\sqrt{-\Delta_{g}}\right)$ are both selfadjoint with respect to $g$ ( $\phi$ can always be chosen to be real). Therefore, $\psi_{j}\left(\sqrt{-\Delta_{g}}\right) \circ m_{\phi}=\left(m_{\phi} \circ \psi_{j}\left(\sqrt{-\Delta_{g}}\right)\right)^{*}$.

If $p(x, D)$ is a pseudo-differential operator with symbol $p(x, \xi)$, there is an explicit integral expression for the action of $p(x, D)^{*}$ in local coordinates:

$$
p(x, D)^{*} f(x)=\frac{1}{(2 \pi)^{n}} \int_{\mathbb{R}^{n}} \int_{\mathbb{R}^{n}} p(y, \xi)^{*} f(y) e^{i(x-y) \cdot \xi} d y d \xi,
$$


where the integration must be performed in the given order (or else consider the Fourier Transform acting on distributions). Here, $p(y, \xi)^{*}$ is not a symbol in the usual sense. These more general objects are sometimes called amplitudes and are very useful in treating adjoints and products of pseudo-differential operators (see [Tr] and [Tay4, for example). Therefore, we can rewrite $\psi_{j}\left(\sqrt{-\Delta_{g}}\right) \circ m_{\phi} f(x)$ as

$$
\begin{aligned}
& \left(m_{\phi} \circ \psi_{j}\left(\sqrt{-\Delta_{g}}\right)\right)^{*} f(x) \\
& \quad=\frac{1}{(2 \pi)^{n}} \int_{\mathbb{R}^{n}} \int_{\mathbb{R}^{n}} \sigma\left(m_{\phi} \circ \psi_{j}\left(\sqrt{-\Delta_{g}}\right)\right)^{*}(y, \xi) f(y) e^{i(x-y) \cdot \xi} d y d \xi \\
& \quad=\frac{1}{(2 \pi)^{n}} \int_{\mathbb{R}^{n}} \int_{\mathbb{R}^{n}} \sigma\left(m_{\phi} \circ \psi_{j}\left(\sqrt{-\Delta_{g}}\right)\right)(y, \xi) f(y) e^{i(x-y) \cdot \xi} d y d \xi,
\end{aligned}
$$

because the symbols are all real.

Remark 4.8. In general, the symbol of the product $q(x, D)$ of two operators $p_{j}(X, D), j=1,2$, is given by an infinite expression of the form

$$
q(x, \xi) \sim \sum_{|\alpha| \geq 0} \frac{i^{|\alpha|}}{\alpha !} D_{\xi}^{\alpha} p_{1}(x, \xi) D_{x}^{\alpha} p_{2}(x, \xi) .
$$

By contrast, $q(x, \xi)$ becomes simply the product of the symbols, whenever $p_{1}(x, \xi)$ is actually independent of $\xi$ or $p_{2}(x, \xi)$ is independent of $x$.

In particular, since $\sigma\left(m_{\phi}\right)(x, \xi)=\phi(x)$ does not depend on $\xi$,

$$
\sigma\left(m_{\phi} \circ \psi_{j}\left(\sqrt{-\Delta_{g}}\right)\right)(y, \xi)=\phi(y) \sigma\left(\psi_{j}\left(\sqrt{-\Delta_{g}}\right)\right)(y, \xi) .
$$

So finally, after identifying $f$ and $\phi$ with their expression in local coordinates,

$$
\begin{aligned}
\| \psi_{j}\left(\sqrt{-\Delta_{g}}\right) & \circ m_{\phi} f \|_{M_{q}^{p}} \\
& =\frac{1}{(2 \pi)^{n}}\left\|\int_{\mathbb{R}^{n}} \int_{\mathbb{R}^{n}} \phi(y) \sigma\left(\psi_{j}\left(\sqrt{-\Delta_{g}}\right)\right)(y, \xi) f(y) e^{i(\cdot-y) \cdot \xi} d y d \xi\right\|_{M_{q}^{p}} \\
& \leq \frac{1}{(2 \pi)^{n}}\|\phi\|_{L^{\infty}}\left\|\psi_{j}\left(\sqrt{-\Delta_{g}}\right) f\right\|_{M_{q}^{p}},
\end{aligned}
$$

which proves (4.27).

We split the proof of Theorem (4.7) into two distinct parts. Let $A$ be the operator 11 obtained by transporting $\sqrt{-\Delta_{g}}$ to $\mathbb{R}^{n}$.

Lemma 4.9. Let $s \in \mathbb{R}, 1<q \leq p<\infty, r \in[1, \infty]$. For every $f \in \mathcal{E}^{\prime}\left(\mathbb{R}^{n}\right)$, there exists a constant $C=C(s, p, q, r)$ such that

$$
\left\|\left\{2^{j s}\left\|\psi_{j}(A) f\right\|_{M_{q}^{p}\left(\mathbb{R}^{n}\right)}\right\}\right\|_{\ell^{r}} \leq C\left\|\left\{2^{j s}\left\|\psi_{j}(D) f\right\|_{M_{q}^{p}\left(\mathbb{R}^{n}\right)}\right\}\right\|_{\ell^{r}} .
$$

Proof. The proof uses arguments very similar to those in Section 4.1. In fact, the expansion (4.25) is basically of the same type as (4.9) if we set $\delta=2^{-j}$ and replace $\rho$ with $\psi$; that is,

$$
\sigma\left(\psi\left(2^{-j} A\right)\right) \sim \sum_{k \geq 0} 2^{-2 j k} \psi^{(2 k)}\left(2^{-j}|\xi|_{x}\right) p_{k}\left(x, 2^{-j} \xi\right),
$$

with $p_{k}$ a classical symbol of order $k$. Once again, note that differentiating with respect to $\xi$ produces powers of $2^{-j}$, while differentiating with respect to $x$ does not change the asymptotic behavior as $j \rightarrow \infty$, because these are all symbols of type

\footnotetext{
11 This operator is clearly not uniquely determined.
} 
$(1,0)$ and $2^{-j}$ appears in combination with $\xi$. Therefore, the term of order $k$ in (4.34) is uniformly bounded in $S_{1,0}^{-k}$, with respect to $j$, and $O\left(2^{j(l-2 k)}\right)$ in $S_{1,0}^{-(k-l)}$ for $0 \leq l \leq k$. To prove the lemma, it is then enough to show that each term in the above expansion is a compactly-supported function and that the $\xi$-support is still essentially a dyadic shell.

We can assume that $g^{i j}$ is the identity outside a compact set in $\mathbb{R}^{n}$. The usual argument shows that $\operatorname{Supp}\left\{\psi^{(2 k)}\left(2^{-j}|\xi|_{x}\right)\right\}$ is covered by a fixed number of shells $D_{m}$, so that again, as in (4.13), one writes

$$
\psi^{(2 k)}\left(2^{-j}|\xi|_{x}\right) p_{k}\left(x, 2^{-j} \xi\right)=\sum_{j=l-N}^{l+N} \psi^{(2 k)}\left(2^{-j}|\xi|_{x}\right) p_{k}\left(x, 2^{-j} \xi\right) \psi_{l}(\xi),
$$

with $N$ independent of $x$ and $j$.

Let $Q_{k}^{j}$ be the operator with symbol $\psi^{(2 k)}\left(2^{-j}|\xi|_{x}\right) p_{k}\left(x, 2^{-j} \xi\right)$. Then, an analysis parallel to (4.14) and (4.15) yields

$$
\left\|\left\{2^{j s}\left\|Q_{k}^{j} f\right\|_{M_{q}^{p}}\right\}\right\|_{\ell^{r}} \leq C(s, p, q, r)\|f\|_{N_{p, q, r}^{s}} .
$$

Again, we obtain a bound on any finite number of terms in the expansion (4.34). To conclude the proof, it is therefore sufficient to show that the norm of the remainder is weak enough, i.e., we set

$$
\sigma\left(\psi\left(2^{-j} A\right)\right)=\sum_{0 \leq k \leq L-1} 2^{-2 j k} \psi^{(2 k)}\left(2^{-j}|\xi|_{x}\right) p_{k}\left(x, 2^{-j} \xi\right)+r_{L}\left(x, \xi, 2^{-j}\right),
$$

and we prove that

$$
\left\|\left\{2^{j s}\left\|r_{L}\left(x, D, 2^{-j}\right) f\right\|_{M_{q}^{p}}\right\}\right\|_{\ell^{r}} \leq C(s, p, q, r, L)\|f\|_{N_{p, q, r}^{s}},
$$

for $L$ large enough. But, since $2^{j s} r_{L}\left(x, \xi, 2^{-j}\right)$ is $O\left(2^{j(s+l-2 L)}\right)$ in $S_{1,0}^{l-L}$ for $0 \leq l \leq$ $L$, 4.38) follows from estimates similar to (4.20) and (4.21).

The opposite inequality is more complex. Informally, it is true because the principal symbol of $\psi\left(2^{-j} \sqrt{-\Delta_{g}}\right), \psi\left(2^{-j}|\xi|_{x}\right)$, enjoys properties similar to those of $\psi\left(2^{-j} \xi\right)$. However, a careful analysis of the terms of lower order is necessary.

Lemma 4.10. Under the hypotheses of Lemma 4.9, it also follows that

$$
\left\|\left\{2^{j s}\left\|\psi_{j}(D) f\right\|_{M_{q}^{p}\left(\mathbb{R}^{n}\right)}\right\}\right\|_{\ell^{r}} \leq C\left\|\left\{2^{j s}\left\|\psi_{j}(A) f\right\|_{M_{q}^{p}\left(\mathbb{R}^{n}\right)}\right\}\right\|_{\ell^{r}}
$$

Proof. The basic idea is somehow to "reverse" (4.35). For each fixed $x,\left\{\psi_{j}\left(|\xi|_{x}\right)\right\}$ is a partition of unity. Therefore,

$$
\psi_{j}(\xi)=\sum_{l=j-N(x)}^{l=j+N(x)} \psi_{j}(\xi) \psi_{l}\left(|\xi|_{x}\right) .
$$

In principle, $N(x)$ varies with $x \in \mathbb{R}^{n}$. However, since the metric $g^{i j}$ is the identity outside a compact set, an argument similar to that leading to 4.13) shows $N(x) \leq$ $N$ for some fixed number $N$. We would then like to replace symbols by operators and use uniform estimates on the norm of $\psi_{j}(D)$. Using the notation introduced in the previous lemma, let us call $Q_{0}^{l}$ the operator whose symbol is $\psi_{l}\left(|\xi|_{x}\right)$. Unfortunately, $\psi_{j}(\xi) \psi_{l}\left(|\xi|_{x}\right)$ is not the symbol of the product $\psi_{j}(D) \circ Q_{0}^{l}$, but one can get around 
this problem again by using amplitudes instead of symbols. Since $\psi_{j}(D)$ is selfadjoint (with respect to the Euclidean metric on $\mathbb{R}^{n}$ ), one deduces similarly to (4.30):

$$
\begin{aligned}
\psi_{j}(D) \circ Q_{0}^{l} f(x) & =\left(Q_{0}^{l *} \circ \psi_{j}(D)\right)^{*} f(x) \\
& =\frac{1}{(2 \pi)^{n}} \int_{\mathbb{R}^{n}} \int_{\mathbb{R}^{n}} \sigma\left(Q_{0}^{l *} \psi_{j}(D)\right)(y, \xi) e^{i(x-y) \cdot \xi} f(y) d y d \xi \\
& =\frac{1}{(2 \pi)^{n}} \int_{\mathbb{R}^{n}} \int_{\mathbb{R}^{n}} \psi_{j}(\xi) \sigma\left(Q_{0}^{l *}\right)(y, \xi) e^{i(x-y) \cdot \xi} f(y) d y d \xi,
\end{aligned}
$$

where the last line follows from (4.31), this time because the symbol of $\psi_{j}(D)$ is independent of $x$. Moreover,

$$
\sigma\left(Q_{0}^{l *}\right)(y, \xi) \sim \psi_{l}\left(|\xi|_{y}\right),
$$

up to a residua $12 r_{l}^{1}(x, \xi)$, which is $O\left(2^{-l}\right)$ in $S_{1,0}^{0}$.

Using (4.40) and (4.41) along with the above result, we finally get

$$
\begin{aligned}
\psi_{j}(D) f(x) & =\frac{1}{(2 \pi)^{n}} \int_{\mathbb{R}^{n}} \int_{\mathbb{R}^{n}} \psi_{j}(\xi) e^{i(x-y) \cdot \xi} f(y) d y d \xi \\
& =\sum_{l=j-N}^{l=j+N} \frac{1}{(2 \pi)^{n}} \int_{\mathbb{R}^{n}} \int_{\mathbb{R}^{n}} \psi_{j}(\xi) \psi_{l}\left(|\xi|_{x}\right) e^{i(x-y) \cdot \xi} f(y) d y d \xi \\
& =\sum_{l=j-N}^{l=j+N} \frac{1}{(2 \pi)^{n}} \int_{\mathbb{R}^{n}} \int_{\mathbb{R}^{n}} \psi_{j}(\xi)\left[\sigma\left(Q_{0}^{l *}\right)(y, \xi)-r_{l}^{1}(y, \xi)\right] e^{i(x-y) \cdot \xi} f(y) d y d \xi \\
& =\psi_{j}(D) \circ Q_{0}^{l} f(x)-\psi_{j}(D) \circ \tilde{r}_{l}^{1}(x, D) f(x) .
\end{aligned}
$$

By (4.34) replacing $Q_{0}^{l}$ with $\psi_{l}(A)$ introduces additional lower-order terms. In any case, we have

$$
\left\|\psi_{j}(D) f\right\|_{M_{q}^{p}} \leq \sum_{l=j-N}^{l=j+N}\left\|\psi_{l}(A) f\right\|_{M_{q}^{p}}+\left\|R^{1}\left(\cdot, D, 2^{-j}\right) f\right\|_{M_{q}^{p}},
$$

as $\psi_{j}(D)$ is bounded uniformly in $j$ in $O P S_{1,0}^{0}$, where $R^{1}$ is obtained by collecting all the terms of lower order. Note that $R^{1}\left(x, \xi, 2^{-j}\right)$ still has compact support essentially "centered" around the support of $\psi_{l}\left(|\xi|_{x}\right)$ and, as before, $\left\{2^{j} R^{1}\left(x, \xi, 2^{-j}\right)\right\}_{j \geq 0}$ is bounded in $S_{1,0}^{0}$. Hence, the same analysis performed in (4.40) through (4.42) can be carried out here. There is a small complication, namely $R^{1}\left(x, \xi, 2^{-j}\right) \psi_{l}\left(|\xi|_{x}\right)$ is not the symbol of the product of the operators $R^{1}\left(x, D, 2^{-j}\right)$ and $Q_{0}^{l}$ no matter what the order of the product is; so the equivalent of (4.41) is true $\bmod S_{1,0}^{-2}$. But the error contributes only to the calculation at the next order.

After $L$ steps, we obtain the following estimate:

$$
\left\|\psi_{j}(D) f\right\|_{M_{q}^{p}} \leq \sum_{l=j-M_{L}}^{l=j+M_{L}}\left\|\psi_{l}(A) f\right\|_{M_{q}^{p}}+\left\|R^{L}\left(\cdot, D, 2^{-j}\right) f\right\|_{M_{q}^{p}},
$$

where $M_{L}$ depends on $L$, but not on $j$, and $R^{L}\left(x, \xi, 2^{-j}\right)$ is $O\left(2^{-j L}\right)$ in $S_{1,0}^{0}$. As in the previous lemma, it is enough to establish that the norm of the residual is sufficiently weak to conclude the proof.

\footnotetext{
${ }^{12}$ It is precisely $\sum_{|\alpha|>0}\left(i^{|\alpha|} / \alpha !\right) D_{\xi} D_{x} \psi\left(2^{-l}|\xi|_{x}\right)$.
} 
First, we show that $\left\|R^{L}\left(\cdot, D, 2^{-j}\right) f\right\|_{M_{q}^{p}}$ is weaker than a Sobolev norm with an appropriate large negative index. Sobolev norms are a natural choice, because $\langle\xi\rangle$ has roughly size $2^{j}$ on $\operatorname{Supp} R_{L}$.

Now, $R^{L}\left(x, D, 2^{-j}\right) f \in \mathcal{S}$, since $R^{L}\left(x, D, 2^{-j}\right)$ is smoothing and $f \in \mathcal{E}^{\prime}$; so Hölder's inequality gives

$$
\left\|R^{L}\left(\cdot, D, 2^{-j}\right) f\right\|_{M_{q}^{p}} \leq \sup _{\substack{0<R \leq 1 \\ x_{0} \in \mathbb{R}^{n}}}\left[R^{n / p-n / q} \operatorname{Vol}\left(B\left(x_{0}, R\right)\right)^{1 / t}\left\|R^{L}\left(\cdot, D, 2^{-j}\right) f\right\|_{L^{m}}\right]
$$

with $m \geq q$ and $t=m q /(m-q)$. If, in addition, $m$ is chosen greater than $p$, then $n / p-n / q+n / t=n / p-n / q+n / q-n / m=n / p-n / m \geq 0$, and (4.44) becomes simply

$$
\left\|R^{L}\left(\cdot, D, 2^{-j}\right) f\right\|_{M_{q}^{p}} \leq C(n, m)\left\|R^{L}\left(\cdot, D, 2^{-j}\right) f\right\|_{L^{m}},
$$

since $\operatorname{Vol}\left(B\left(x_{0}, R\right)\right) \sim R^{n}$.

Recall that $\left\{2^{j L} R^{L}\left(x, \xi, 2^{-j}\right)\right\}$ is bounded in $S_{1,0}^{0}$, so that

$$
\begin{aligned}
\left\|R^{L}\left(\cdot, D, 2^{-j}\right) f\right\|_{L^{m}} & =\left\|\mathcal{F}_{\xi}^{-1}\left(R^{L}\left(\cdot, \xi, 2^{-j}\right) \hat{f}\right)\right\|_{L^{m}} \\
& \sim \| \mathcal{F}_{\xi}^{-1}\left(\langle\xi\rangle^{-L} 2^{L j} R^{L}\left(\cdot, \xi, 2^{-j}\right) \hat{f} \|_{L^{m}}\right. \\
& \sim\left\|2^{(L-\alpha) j} R^{L}\left(\cdot, D, 2^{-j}\right)(I-\Delta)^{\alpha-L} f\right\|_{L^{m}} \\
& \leq \tilde{C} 2^{-\alpha j}\left\|(I-\Delta)^{\alpha-L} f\right\|_{L^{m}} \\
& =C 2^{-\alpha j}\|f\|_{H_{m}^{\alpha-L}}
\end{aligned}
$$

where $H_{m}^{\alpha-L}$ is the $L^{m}$-based Sobolev space of order $\alpha-L$. We select $\alpha$ such that $|s|<\alpha<|s|+1$, and obtain

$$
\left\|\left\{2^{j s}\left\|R^{L}\left(\cdot, D, 2^{-j}\right) f\right\|_{M_{q}^{p}}\right\}\right\|_{\ell^{r}} \leq C\left\|\left\{2^{(s-\alpha) j}\right\}\right\|_{\ell^{r}}\|f\|_{H_{m}^{\alpha-L}} .
$$

It remains to show that

$$
\|f\|_{H_{m}^{\alpha-L}} \leq C\left\|\left\{2^{j s}\left\|\psi_{j}(D) f\right\|_{M_{q}^{p}}\right\}\right\|_{\ell^{r}} .
$$

But (see Tay1], for example)

$$
\|f\|_{H_{m}^{\alpha-L}\left(\mathbb{R}^{n}\right)} \approx\left[\int_{\mathbb{R}^{n}}\left(\sum_{j=0}^{\infty} 4^{j(\alpha-L)}\left|\psi_{j}(D) f\right|^{2}\right)^{\frac{m}{2}} d x\right]^{\frac{1}{m}} .
$$

Then pick $L>2|s|+1+n(1 / q-1 / m)$ :

$$
\begin{aligned}
& \|f\|_{H_{m}^{\alpha-L}} \leq C \sum_{j=0}^{\infty} 2^{j(\alpha-L)}\left\|\psi_{j}(D) f\right\|_{L^{m}} \\
& \quad \leq C\left\|\left\{2^{j(\alpha-L-s)+j n(1 / q-1 / m)}\right\}\right\|_{\ell^{\frac{r}{r-1}}}\left\|\left\{2^{j s-j n(1 / q-1 / m)}\left\|\psi_{j}(D) f\right\|_{L^{m}}\right\}\right\|_{\ell^{r}},
\end{aligned}
$$

by the triangle and Hölder's inequalities. Finally, since $\psi_{j}(D) f$ is rapidly decreasing and $m \geq p \geq q$, by Berstein's inequality

$$
\begin{aligned}
2^{-j n(1 / q-1 / m)}\left\|\psi_{j}(D) f\right\|_{L^{m}\left(\mathbb{R}^{n}\right)} & \leq C\left\|\psi_{j}(D) f\right\|_{L^{q}\left(\mathbb{R}^{n}\right)} \leq C\left\|\psi_{j}(D) f\right\|_{M_{q}^{q}\left(\mathbb{R}^{n}\right)} \\
& \leq C\left\|\psi_{j}(D) f\right\|_{M_{q}^{p}\left(\mathbb{R}^{n}\right)} .
\end{aligned}
$$

The lemma now follows from (4.43). 
To show that the $L^{q}$-norm is comparable to the Morrey norm in $M_{q}^{q}$ for a rapidly decreasing function $g$, pick a ball of large radius around the origin, say $B(0, K)$, where the radius is so big that $\|g\|_{L^{q}\left(B(0, K)^{\mathrm{c}}\right)}$ is smaller than any given $\epsilon$. Then, cover $B(0, K)$ with a finite number of balls of given radius $R \in(0,1)$. Recall that, in general, $g \in M_{q}^{p}$ is only locally in $L^{q}$. Note also that, in the case of $g=\psi_{j}(D) f$, because $\breve{\psi}_{j}$ are concentrated on balls of shrinking size, the radius $K$ can be taken to be independent of $j$, yielding uniform estimates.

Remark 4.11. As observed in [SeSo, one can replace $\sqrt{-\Delta_{g}}$ in (4.24) with any first-order elliptic (classical) pseudo-differential operator $P$ that is positive and selfadjoint on $L^{2}(M)$.

We conclude this section with one final intrinsic characterization of BM spaces. Recall $N_{p, q, r}^{s}\left(\mathbb{R}^{n}\right)$ can be characterized as a real interpolation space (Proposition [2.6):

$$
N_{p, q, r}^{s}\left(\mathbb{R}^{n}\right)=\left((I-\Delta)^{-s_{1} / 2} M_{q}^{p}\left(\mathbb{R}^{n}\right),(I-\Delta)^{-s_{2} / 2} M_{q}^{p}\left(\mathbb{R}^{n}\right)\right)_{r, \theta} .
$$

It is reasonable to expect a similar property will hold for $N_{p, q, r}^{s}(M)$, given its definition in terms of local charts. Observe that we can form the interpolation space $\left(\left(I-\Delta_{g}\right)^{-s_{1} / 2} M_{q}^{p}(M),\left(I-\Delta_{g}\right)^{-s_{2} / 2} M_{q}^{p}(M)\right)_{r, \theta}$. It is only a matter of recognizing this space appropriately.

Proposition 4.12. For $s \in \mathbb{R}, r \in[1, \infty]$ and $1<q \leq p<\infty$,

$$
N_{p, q, r}^{s}(M)=\left(\left(I-\Delta_{g}\right)^{-s_{1} / 2} M_{q}^{p}(M),\left(I-\Delta_{g}\right)^{-s_{2} / 2} M_{q}^{p}(M)\right)_{r, \theta},
$$

where $s_{1} \neq s_{2}, \theta \in(0,1)$, and $s=(1-\theta) s_{1}+\theta s_{2}$.

Proof. Suppose first $f \in N_{p, q, r}^{s}(M)$. Because of Definition 4.5 identifying $f$ with its local image, we can assume that $f \in N_{p, q, r}^{s}\left(\mathbb{R}^{n}\right)$ is compactly supported. So, by (4.52) we can write $f$ as a sum of two functions $f_{j}(t) \in(I-\Delta)^{-s_{j} / 2} M_{q}^{p}\left(\mathbb{R}^{n}\right)$, $j=1,2, t \in[0, \infty)$, and

$$
\|f\|_{N_{p, q, r}^{s}}=\sup _{\left(f_{1}, f_{2}\right)} \sum_{j=1,2}\left\|t^{\zeta_{j}}\right\| f_{j}(t)\left\|_{(I-\Delta)^{-s_{j} / 2} M_{q}^{p}\left(\mathbb{R}^{n}\right)}\right\|_{L^{r^{*}}}
$$

where the supremum is over all possible representations of $f$ with $\zeta_{j}$ real numbers such that $\zeta_{1}(1-\theta)+\zeta_{2} \theta=0$ (see [Trieb1], p. 35). Since this representation actually characterizes real interpolation spaces, it is enough to show that (4.54) gives equivalent norms for different choices of coordinate systems on $M$. In fact, $(I-\Delta)$ can be replaced by $\left(I-\Delta_{g}\right)$, because the operator obtained by transporting $\Delta_{g}$ to $\mathbb{R}^{n}$ is still elliptic and negative-definite. But, clearly the topology of $(I-\Delta)^{-s_{j} / 2} M_{q}^{p}\left(\mathbb{R}^{n}\right)$ is invariant under the action of a diffeomorphism $\chi$ in the same way as $M_{q}^{p}$ is.

The reverse inclusion follows immediately from (4.52) by means of partitions of unity, because of Definitions 4.2 and 4.5 .

\section{Applications to Semi-linear Parabolic Equations}

In [KY], Kozono and Yamazaki studied solutions to a class of semi-linear parabolic equations with initial data in certain BM spaces on $\mathbb{R}^{n}$. This class includes the Navier-Stokes equation (NS for short). 
Independently, Cannone Can developed the notion of adapted spaces for NS, combining Littlewood-Paley theory with Bony's paraproduct. This concept gives a rather general criterion under which existence and uniqueness can be established by simple contraction mapping or Picard iteration. Then, Karch $\underline{\mathrm{Kr}}$ pushed Cannone's approach further, in a more abstract setting, by introducing the spaces $B E^{\alpha}$.

In this section, we compare and generalize these apparently different results, using the material previously developed. In particular, all the conclusions obtained in $[\mathrm{KY}]$ can be recovered from the more general theory of adapted and $B E^{\alpha}$ spaces. We also study the same class of equations on compact manifolds. As pointed out by Ebin and Marsden [EM], the Navier-Stokes equation must be modified to take curvature into account. Sections 5.1-5.3 recall known results and Sections 5.45.5 present new results.

5.1. Background. Semi-linear parabolic equations on $\mathbb{R}^{n}$ can be fundamentally treated using methods for ordinary differential equations. In particular, an Initial Value Problem (IVP for short) can be recast in the form of an integral equation with coefficients in a Banach space. This formulation suggests using fixed-point theorems.

Let $A$ be a positive semi-definite operator on a Banach space $X$, such that $-A$ generates a $C^{0}$ semigroup $U(t), t \geq 0$, on $X$. Consider the following IVP:

$$
u_{t}+A u=F(u), \quad u(0)=a,
$$

where $F:[0, T] \times \mathcal{O} \rightarrow X$ is continuous, $\mathcal{O}$ is an open subset of $X$, and $a \in X$. We use the notation $u(t)(x)=u(t, x), x \in X$.

Example 5.1. Take $A=-\Delta, F(u)=\mathbb{P} \operatorname{div}(u \otimes u), X=L^{2}, \mathcal{O}=H^{s}, s>5 / 2$, $n=3$. Then (5.1) is the classical formulation of NS (see Section 5.3 ).

In this abstract setting, boundary conditions or decay at infinity for unbounded domains are implicit in the choice of the function space $X$.

Definition 5.2. A function $u \in C([0, T], X)$ that satisfies the integral equation

$$
u(t)=U(t) a+\int_{0}^{t} U(t-s) F(u(s)) d s, \quad 0 \leq t \leq T,
$$

is called a mild solution to (5.1).

In general, the integral formulation is weaker than the initial IVP, because we only need $U(t-\cdot) F\left(u(\cdot) \in L^{1}([0, T], X)\right.$. This is especially true if $U(t)$ has smoothing properties as in the heat semigroup. However, if $F$ is Lipschitz, $X$ is reflexive, and $a \in \mathcal{D}(A)$, then any mild solution to (5.2) is actually a strong solution to (5.1). Moreover, it is a theorem of J. M. Ball that, in this case, every weak solution is unique and it is, in fact, a mild solution (see $[\mathrm{Pa}]$ ).

Unfortunately, this result does not help in establishing uniqueness of Leray-Hopf solutions for NS, since we do not know if $F$ is globally Lipschitz. But it motivates looking for mild solutions for "rough" initial data, even when the data do not belong to $L^{2}$ by means of splitting techniques (see, for example, [Lio]).

Note that the heat semigroup is not strongly continuous in these spaces. Hence, not all mild solutions are also strong solutions, and the initial value is attained only in a weak sense, which will be made precise.

Typically, the data are distributions taken from a scale of spaces and there exists a range of indices bounded from below for which the Banach contraction theorem 
applies to the integral equation, yielding local-in-time, unique solutions. The lower bound corresponds to the so-called limit spaces, characterized by invariance of the norm under the natural scaling of the corresponding equation. In this case, it is necessary to impose an extra "smallness" condition on the initial data and (global) existence is then obtained by direct analysis of Picard iterates. But uniqueness holds only in an appropriate subspace of the solution space.

There is an extensive literature 13 on the Navier-Stokes equation with initial data in $L^{p}$ (e.g. [FK], Ka1]) and Morrey Spaces (e.g. [Fe, [Ka2], Ka3], GM], GMO, Tay3 ). Hydrodynamics in Morrey spaces is interesting for several reasons. For example, the theory extends to include Radon measures $\mu \in \tilde{\mathcal{M}}^{p}$, which can model vorticity supported on singular sets of $\mathbb{R}^{n}$, e.g. vortex rings and vortex sheets. Also, in the specific context of adapted spaces, we have the following result (in dimension $n=3)$.

Proposition 5.3 ([LMR] $)$. Suppose $E$ is an adapted space to NS such that

(1) $E \hookrightarrow L_{l o c}^{2}$,

(2) for all $\lambda>0$, the dilation $f \rightarrow f_{\lambda}$ is continuous on $E$.

Then $E \hookrightarrow M_{2}^{3}$ and $\lim _{t \rightarrow 0} \sqrt{t}\left\|e^{t \Delta} f\right\|_{L^{\infty}}=0$.

Recall that an adapted space $E$ is characterized by the property

$$
\left\|\psi_{j}(D)(f g)\right\|_{E} \leq \eta_{j}\|f\|_{E}\|g\|_{E}, \quad \forall j \in \mathbb{Z},
$$

where

$$
\sum_{j \in \mathbb{Z}} 2^{-|j|} \eta_{j}<\infty
$$

The above conditions ensure that the right-hand side of (5.2) defines a Lipschitz map locally-in-time and, hence, that a short-time, unique solution to NS exists.

Consequently, analysis in adapted spaces actually concerns regularity results, once existence of solutions in Morrey spaces is established.

As we remarked in the Introduction, choosing BM spaces, which are of Besovtype, over Morrey spaces has additional advantages. First of all, there is a better pseudo-differential and para-differential calculus, especially with respect to pointwise multiplication. Secondly, BM spaces of negative index contain distributions more singular than Radon measures. For example, p.v. $(1 / x) \in \mathcal{N}_{1,1, \infty}^{0}(\mathbb{R})$, so that $\left(0, \ldots, 0\right.$, p.v. $\left.\left(1 / x_{1}\right)\right) \in \mathcal{N}_{n, 1, \infty}^{0}\left(\mathbb{R}^{n}\right) \subset \mathcal{N}_{p, p / n, \infty}^{n / p-1}\left(\mathbb{R}^{n}\right)$ as long as $p>n$ (see $[\mathrm{KY}]$, p. 969). Note that Theorem 5.12 below applies with $a(x)=\delta\left(0, \ldots, 0\right.$, p.v. $\left.\left(1 / x_{1}\right)\right)$ as initial data, provided $\delta$ is sufficiently small.

As mentioned in Section 2.2. Koch and Tataru have proved existence and uniqueness of global solutions with data small in $\mathrm{BMO}^{-1}$. Their result does not include Theorem 5.12 entirely, as one can take data small in $\mathcal{N}_{p, 1, \infty}^{n / p-1}$, as long as $p>n$, and $\mathcal{N}_{p, 1, \infty}^{n / p-1} \nsubseteq \mathrm{BMO}^{-1}$ (see also [CX]).

In $[\mathrm{KY}$, Kozono and Yamazaki focus on scalar perturbation of the heat equations, and the Navier-Stokes equation. However, at least as far as existence and uniqueness are concerned, the proof applies to systems of equations, as long as the non-linear part satisfies certain homogeneity constraints.

We first recall Kozono and Yamazaki's results and then discuss some generalizations.

\footnotetext{
${ }^{13}$ For an overview of recent results we refer to [Ya].
} 
5.2. Semi-linear heat equations. We consider the Initial Value Problem:

$$
\left\{\begin{array}{l}
\partial_{t} u(t, x)=\Delta u(t, x)+f(u(t, x)), \\
u(0, x)=a(x),
\end{array}\right.
$$

where $u:(0,+\infty) \times \mathbb{R}^{n} \rightarrow \mathbb{C}$ and $f: \mathbb{C} \rightarrow \mathbb{C}$. We assume that $f$ is a locally Lipschitz function satisfying

$$
|f(z)-f(w)| \leq C|z-w|\left(1+|z|^{\gamma-1}+|w|^{\gamma-1}\right),
$$

for some $\gamma>1$. Equations of this form arise, for example, in mathematical biology and control theory $[\mathrm{Wu}]$.

One of the main results in $[\mathrm{KY}]$ is the following theorem.

Theorem 5.4 ([KY). Suppose that $f$ satisfies (5.6) and assume $p, q, s$ are numbers such that

$$
\left\{\begin{array}{l}
\gamma \leq q \leq p \\
n(\gamma-1)<2 p \\
-2 / \gamma<s<0 \\
s \geq n / p-2 /(\gamma-1) .
\end{array}\right.
$$

Then, there exist $\delta, K>0$ such that for every $a \in N_{p, q, \infty}^{s}$ satisfying

$$
\limsup _{j \rightarrow \infty} 2^{j s}\left\|\psi_{j}(D) a\right\|_{M_{q}^{p}}<\delta,
$$

there are $T>0$ and a mild solution $u(t, x)$ of (5.5) on $[0, T) \times \mathbb{R}^{n}$ such that

(1) $\sup _{0<t \leq T} t^{-s / 2}\|u(t, \cdot)\|_{M_{q}^{p}} \leq K$,

(2) $u(t, \cdot) \underset{t \rightarrow 0^{+}}{\rightarrow}$ a in the weak-* topology of $B_{\infty, \infty}^{s-n / p}$ (as dual of $B_{1,1}^{n / p-s}$ ),

(3) $u \in L^{\infty}\left((0, T), N_{p, q, r}^{s}\right)$,

(4) $u(t, x)$ is continuously differentiable in $t$ and twice continuously differentiable in $x$ on $(0, T) \times \mathbb{R}^{n}$.

Above, $T$ is the time of existence of the solution, which is not maximal in general. The condition imposed on $a$ (the "smallness" of the initial data hinted at before) is fundamentally a bound on an appropriate auxiliary norm, as will become clear in the course of the proof. It controls the local singularity of $a$ (recall $s$ is negative). It follows from a theorem of F. Weissler [We that, because $N_{p, \gamma, \infty}^{n / p-2 /(\gamma-1)}$ contains the Lorentz space $L^{n(\gamma-1) / 2, \infty}$ for the range of indices above, this extra hypothesis cannot be eliminated (see [KY], pp. 991-992). However, it is redundant for $s>$ $n / p-2 /(\gamma-1)$, as we will show later.

Remark 5.5. (5.8) is also the analogue of the condition $a \in \stackrel{\circ}{M_{1}^{n}}$ in Theorem 4.3 of Tay3, where $\stackrel{\circ}{M_{1}^{n}}$ is the set of functions $u$ for which

$$
\nu_{p q}(u)=\limsup _{R \rightarrow 0} R^{n / p-n / q}\|u\|_{L^{q}(B(0, R))}=0 .
$$

Note further that, if $u(t, x)$ is a solution to the problem (5.5), then, for each positive $\lambda, \lambda^{2 /(\gamma-1)} u\left(\lambda^{2} t, \lambda x\right)$ is also a solution. So, the norm of the homogeneous space $\mathcal{N}_{p, q, \infty}^{n / p-2 /(\gamma-1)}$ is exactly the one invariant under such scaling, that is, this space is a limit space and $n / p-2 /(\gamma-1)$ is the critical index. Accordingly, one 
does not expect a simple contraction mapping argument to hold and the Picard iteration must be inspected directly.

We sketch the proof of the theorem, since similar arguments will be used later. The main tool consists of the following estimates on the heat semigroup.

Lemma 5.6 ([KY]). Let $\ell \geq s$ and $1 \leq q \leq p<\infty$. Then

$$
\begin{aligned}
\left\|e^{t \Delta} u\right\|_{N_{p, q, \infty}^{\ell}} & \leq C\left(1+t^{(s-\ell) / 2}\right)\|u\|_{N_{p, q, \infty}^{s}}, \\
\left\|e^{t \Delta} u\right\|_{\mathcal{N}_{p, q, \infty}^{\ell}} & \leq C t^{(s-\ell) / 2}\|u\|_{\mathcal{N}_{p, q, \infty}^{s}} .
\end{aligned}
$$

If $\ell>s$, then also

$$
\begin{aligned}
\left\|e^{t \Delta} u\right\|_{N_{p, q, 1}^{\ell}} & \leq C\left(1+t^{(s-\ell) / 2}\right)\|u\|_{N_{p, q, \infty}^{s}} \\
\left\|e^{t \Delta} u\right\|_{\mathcal{N}_{p, q, 1}^{\ell}}^{\ell} & \leq C t^{(s-\ell) / 2}\|u\|_{\mathcal{N}_{p, q, \infty}^{s}} .
\end{aligned}
$$

The lemma is an easy consequence of the results of Section 3

Lemma 5.7 ( $[\mathrm{KY}])$. Suppose that $1 \leq q \leq p<\infty$ and $\ell>s$. Then there exists a positive constant $A=A(p, q, s, \ell)$ such that, for every $u \in N_{p, q, \infty}^{s}$ and every $B$ such that

$$
A \limsup _{j \rightarrow \infty} 2^{j s}\left\|\psi_{j}(D) u\right\|_{M_{q}^{p}}<B,
$$

there is $T>0$ for which

$$
\sup _{0<t \leq T} t^{(\ell-s) / 2}\left\|e^{t \Delta} u\right\|_{N_{p, q, 1}^{\ell}}<B .
$$

A (modified) proof is given in Section [5.5, in the context of compact manifolds. The integral equation associated to (5.5) is

$$
u(t)=e^{t \Delta} a+\int_{0}^{t} e^{(t-\tau) \Delta} f(u(\tau, \cdot)) d \tau=\mathfrak{N}(u)(t) .
$$

The non-linear map $\mathfrak{N}$ is formally defined by (5.13). In principle, it should act on the space $C\left([0, T], N_{p, q, \infty}^{s}\right)$ of continuous functions from $[0, T]$ to $N_{p, q, \infty}^{s}$. However, as noted before, $e^{t \Delta}$ is not strongly continuous on $N_{p, q, \infty}^{s}$. Therefore, the integral in the equation above must be interpreted as an improper integral, instead of as a well-defined Bochner integral. Likewise, the initial value is attained only in a weak sense; how weak is precisely the content of Part 2 in Theorem 5.4

Picard iterates are defined, as usual, by

$$
\begin{aligned}
& u_{0}(t, x)=e^{t \Delta} a(x), \\
& u_{1}(t, x)=e^{t \Delta} a(x)+\int_{0}^{t} e^{(t-\tau) \Delta} f\left(u_{0}(\tau, x)\right) d \tau, \\
& \vdots \\
& \vdots \\
& u_{j+1}(t, x)=e^{t \Delta} a(x)+\int_{0}^{t} e^{(t-\tau) \Delta} f\left(u_{j}(\tau, x)\right) d \tau .
\end{aligned}
$$

Let also $v_{j}=u_{j}-u_{j-1}$.

Next, we obtain bounds on the non-linear part of (5.13), using (5.6) and inclusion relations for Morrey spaces under pointwise multiplication.

Since $N_{p, q, 1}^{0} \subset M_{q}^{p}$, in view of Lemma [5.7 we introduce an auxiliary function space. 
Definition 5.8. Let $\mathcal{A}=\mathcal{A}_{T}$ be the space of measurable functions u on $(0, T) \times \mathbb{R}^{n}$ such that

$$
\|u\|_{\mathcal{A}}=\sup _{0<t \leq T} t^{-s / 2}\|u(t, \cdot)\|_{M_{q}^{p}}<\infty .
$$

For $s, p, q$ satisfying (5.7), $\mathcal{A}$ is a functional Banach space. Note that, if $u(t)=$ $e^{t \Delta} a$, up to a scaling factor, depending on $T$, (5.15) is nothing but the equivalent norm in $N_{p, q, r}^{s}$ given by Proposition 2.22 .

Then, set $A_{j}=\left\|u_{j}\right\|_{\mathcal{A}}, B_{j}=\left\|v_{j}\right\|_{\mathcal{A}}$.

Lemma 5.9 ([KY] $)$. (1) Under the hypotheses of Theorem 5.4, there exists $C_{0}>0$, independent of $a$, and a time $T \leq 1$ such that

(2) Moreover, there exists $C_{1}>0$ independent of $T$ such that, $\forall j \geq 0$,

$$
A_{j+1} \leq A_{0}+C_{1}\left(A_{j}^{\gamma}+T^{-s \gamma / 2}\right) .
$$

Part 5.9 follows immediately from Lemma 5.7 and the inclusion $N_{p, q, 1}^{0} \subset M_{q}^{p}$. Part 5.9] is, instead, a consequence of Lemma 5.6 and the Lipschitz condition (5.6) on $f$ by means of the inclusion $N_{p / \gamma, q / \gamma, 1}^{n(\gamma-1) / p} \subset N_{p, q, 1}^{0} \subset M_{q}^{p}$. The details of the proof are in [KY].

The lemma implies, in particular, that the sequence $\left\{A_{j}\right\}$ is bounded. As a matter of fact, for $T$ small enough, $A_{j+1}<C_{1} A_{j}^{\gamma}+2 C_{0} \delta$. Set $g_{\delta}(x)=2 C_{0} \delta+C_{1} x^{\gamma}$. Then $g_{\delta}$ has a unique fixed point $\alpha_{\delta}$ with $\alpha_{\delta}>C_{0} \delta>A_{0}$. Furthermore, $\alpha_{\delta} \leq \omega_{\delta}=$ $2 C_{0} \gamma \delta /(\gamma-1)$, which gives $A_{j} \leq \omega_{\delta}$ for all $j$.

An estimate similar to (5.17) can be obtained for $B_{j}$, using again (5.6) and the bound on $A_{j}$.

Lemma $5.10([\mathrm{KY}])$. There exists $C_{2}$ independent of $T \leq 1$ such that the inequality

$$
B_{j+1} \leq C_{2} B_{j}\left(\omega(\delta)^{\gamma-1}+T^{s(1-\gamma) / 2}\right)
$$

holds $\forall j \geq 0$.

At this point, convergence of the series $\sum_{j} B_{j}$ follows easily for $\delta$ and $T$ sufficiently small, which is in turn enough to show $\left\{u_{j}\right\}$ is a Cauchy sequence in $\mathcal{A}$. The limit $u$ is clearly a solution to (5.13). Additionally, $A_{j} \rightarrow\|u\|_{\mathcal{A}}$ so that $\|u\|_{\mathcal{A}} \leq \omega_{\delta}=K$. Part (11) of Theorem [5.4 is now established.

Part (2) of Theorem [5.4 is a consequence of the inclusion

$$
M_{q}^{p} \subset N_{p, q, \infty}^{0} \subset B_{\infty, \infty}^{-n / p} .
$$

In a fashion similar to the proof of Lemma 5.9, it ensues that

$$
u \in \mathcal{A} \Rightarrow v=\int_{0}^{t} e^{(t-\tau) \Delta} f(u(\tau, \cdot)) d \tau \in C\left([0, T), B_{\infty, \infty}^{s-n / p}\right),
$$

where $B_{\infty, \infty}^{s-n / p}$ is given the weak-* topology as dual of $B_{1,1}^{n / p-s}$. As pointed out by Cannone Can, the solution is composed of a trend $w=e^{t \Delta} a$ and a fluctuation $v$, which is more oscillating and better behaved for $t \rightarrow 0^{+}$.

Part (3) of Theorem 5.4 is an immediate consequence of Lemmas 5.7 and 5.9 while part (44) is a standard regularity argument. We refer to [KY] for details. 
It remains to prove uniqueness.

Proposition $5.11(\mathrm{KY})$. Let $f, p, q, s, \gamma$ satisfy the hypotheses of Theorem 5.4. Then, for every $T \in(0, \infty]$ and every $a \in \mathcal{S}^{\prime}$, there is at most one solution $u$ of (5.5) on $(0, T) \times \mathbb{R}^{n}$ such that

(1) $u \in L^{\infty}\left(\left(0, T^{\prime}\right), N_{p, q, \infty}^{s}\right) \cap \mathcal{A}_{T^{\prime}}$ if $T^{\prime}<T$,

(2) $\lim \sup _{T^{\prime} \rightarrow 0}\|u\|_{\mathcal{A}_{T^{\prime}}} \leq K$,

(3) $u(t, \cdot) \underset{t \rightarrow 0^{+}}{\rightarrow}$ a in $\mathcal{S}^{\prime}$.

Proof. Suppose that $u_{1}$ and $u_{2}$ are both solutions to (5.13). It is clearly enough to show that the two solutions agree on a small interval $\left[0, T^{\prime}\right)$. The trends $w_{1}$ and $w_{2}$ solve the same IVP for the heat equation in $\mathcal{S}^{\prime}$, hence they agree. Next, set $\tilde{v}=v_{1}-v_{2}$ and $H\left(T^{\prime}\right)=\|\tilde{v}\|_{\mathcal{A}_{T^{\prime}}} . H\left(T^{\prime}\right)$ satisfies the inequality

$$
\begin{aligned}
H\left(T^{\prime}\right) & \leq C_{2}\left[\frac{1}{2} \sum_{j=1,2}\left(\sup _{0<t \leq T^{\prime}} t^{-s / 2}\left\|u_{j}(t, \cdot)\right\|_{M_{q}^{p}}\right)^{\gamma-1}+\left(T^{\prime}\right)^{s(1-\gamma) / 2}\right] H\left(T^{\prime}\right) \\
& \leq C_{2}\left(K^{\gamma-1}+\left(T^{\prime}\right)^{s(1-\gamma) / 2}\right) H\left(T^{\prime}\right) .
\end{aligned}
$$

But $C_{2} K^{\gamma-1}$ is small for $T^{\prime}$ small enough given the definition of $\omega_{\delta}$ and Lemma 5.7. Hence $H\left(T^{\prime}\right)=0$.

When $s=n / p-2 /(\gamma-1)$, as emphasized earlier, the norm of $\mathcal{N}_{p, q, \infty}^{s}$ is invariant under rescaling. Therefore, one would expect global-in-time existence to hold for this limit case, in particular, in the form of self-similar solutions [KY2]. However, in order to exploit the action of time dilations, a homogeneity constraint needs to be imposed on $f$.

Assume, then, that $f$ satisfies

$$
|f(z)-f(w)| \leq C|z-w|\left(|z|^{\gamma-1}+|w|^{\gamma-1}\right),
$$

and $f(0)=0$, which, in particular, implies

$$
|f(z)| \leq C|z|^{\gamma} \text {. }
$$

This choice gives bounds similar to (5.17) and (5.18) that are actually independent of $T$. In addition, (5.7) becomes

$$
\left\{\begin{array}{l}
\gamma>1+2 / n \\
\gamma \leq q \leq p \\
n(\gamma-1)<2 p<n \gamma(\gamma-1) .
\end{array}\right.
$$

Now take the initial data $a \in \mathcal{N}_{p, q, \infty}^{n / p-2 /(\gamma-1)}$ such that $\|a\|_{\mathcal{N}_{p, q, \infty}^{n / p-2 /(\gamma-1)}} \leq \delta_{0}$ is small enough. This condition is stronger than (5.8), which is only local. Indeed, the extra control on the behavior at large scales is required for the bootstrap argument in time. However, it is not known if it is necessary for existence of global solutions.

Introduce again the space $\mathcal{A}$, defined here as the collection of all $u$ such that

$$
\|u\|_{\mathcal{A}_{T}}=\sup _{t>0} t^{-s / 2}\|u(t, \cdot)\|_{\mathcal{M}_{q}^{p}}<\infty
$$


We have the analogs of Lemma 5.9 and Lemma 5.10

$$
\begin{aligned}
& A_{0} \leq C_{3} \delta, \\
& A_{j+1} \leq A_{0}+C_{4} A_{j}^{\gamma}, \\
& B_{j+1} \leq C_{5} \omega(\delta)^{\gamma-1} B_{j},
\end{aligned}
$$

with $\omega(\delta)=C_{3} \gamma \delta /(\gamma-1)$ for $\delta \in\left[0, \delta_{0}\right]$. As before, 5.25a and 5.25b give uniform bounds on the $A_{j}$, while (5.25c) implies convergence of the series $\sum_{j} B_{j}$. Uniqueness then follows from Proposition 5.11 .

5.3. The Navier-Stokes equation. We consider, now, an Initial Value Problem for the Navier-Stokes system on $\mathbb{R}^{n}$ :

$$
\left\{\begin{array}{l}
\partial_{t} u(t, x)=\Delta u(t, x)-u \cdot \nabla u(t, x)-\nabla p(t, x)+f(t, x), \\
\operatorname{div} u(t, x)=0, \\
u(0, x)=a(x) .
\end{array}\right.
$$

This is a system of $n+1$ equations in the unknown $u$ (the velocity vector field) and $p$ (the pressure scalar field). The initial data $a$ is assumed to be divergence-free and $f$ represents body forces. The first equation is basically conservation of momentum, or Newton's second law, and the second, the continuity equation, is conservation of mass 14

The density $\rho$ and the viscosity coefficient $\nu$ do not appear in the equations, since they are constant and are conventionally set equal to one. As a matter of fact, the methods employed here rely heavily on the smoothing action on the heat semigroup and they are valid only for strictly positive $\nu$.

In order to rewrite (5.26) in integral form, we are going to make some further (mild) assumptions. First of all, if the forces are conservative and admit a potential $g, f=\nabla g$ can be absorbed in the pressure term. Secondly, from a physical point of view, the role of the pressure is to enforce incompressibility. Mathematically, the Hodge decomposition ensures that gradients are orthogonal to divergence-free vector fields. Thus $\mathbb{P}(\nabla p)=0$, where $\mathbb{P}$ is the so-called Leray projection onto the space of divergence-free vector fields, at least when $p$ is sufficiently regular. Finally, note that $u \cdot \nabla u=\operatorname{div}(u \otimes u)$ if $\operatorname{div} u=0$. This last expression is more appropriate when dealing with distributions.

Therefore, (5.26) reduces to

$$
\left\{\begin{array}{l}
\partial_{t} u(t, x)=\Delta u(t, x)-\mathbb{P} \operatorname{div}(u \otimes u)(t, x), \\
u(0, x)=a(x) .
\end{array}\right.
$$

The original system is now in the form of a single (vector) semi-linear parabolic equation.

The non-linear part of (5.27) is the product of a first-order (non-local) operator $P$, and a bilinear form $\mathcal{Q}$ :

$$
\mathbb{P} \operatorname{div}(u \otimes u)=P \mathcal{Q}(u, u) .
$$

In particular, it has a scaling factor $\gamma=3$ (in the notation of the previous section); consequently, one expects to have local-in-time solutions for initial data in $N_{p, q, \infty}^{s}$,

\footnotetext{
${ }^{14}$ For a derivation of these equations we refer to [ChM].
} 
$s \geq n / p-1$, and global-in-time solutions for initial data in $\mathcal{N}_{p, q, \infty}^{n / p-1}$. Kozono and Yamazaki consider the limit case in [KY]

Theorem 5.12 ([KY] $)$. Let $1 \leq q \leq p<\infty$ and $p>n$. Then, there exist $\delta, K>0$ such that, for every $a \in N_{p, q, \infty}^{n / p-1}$ satisfying

$$
\left\{\begin{array}{l}
\lim _{\sup _{j \rightarrow \infty}} 2^{j(n / p-1)}\left\|\psi_{j}(D) a\right\|_{M_{q}^{p}}<\delta, \\
\operatorname{div} a(x)=0,
\end{array}\right.
$$

there are $T>0$ and a mild solution $u(t, x)$ of (5.27) on $[0, T) \times \mathbb{R}^{n}$ such that

(1) $\sup _{0<t \leq T} t^{1 / 2-n / 4 p}\|u(t, \cdot)\|_{M_{2 q}^{2 p}} \leq K$,

(2) $u(t, \cdot) \underset{t \rightarrow 0^{+}}{\longrightarrow}$ a in the weak-* topology of $B_{\infty, \infty}^{-1}$ (as dual of $B_{1,1}^{1}$ ),

(3) $u \in L^{\infty}\left((0, T), N_{p, q, \infty}^{n / p-1}\right)$,

(4) $u \in C^{\infty}\left((0, T) \times \mathbb{R}_{x}^{n}\right)$.

As in the previous section, we look for solutions of the corresponding integral equation

$$
u(t)=e^{t \Delta} a-\int_{0}^{t} e^{(t-\tau) \Delta} \mathbb{P} \operatorname{div}(u \otimes u)(\tau) d \tau=\mathfrak{N}(u)(t) .
$$

Along with Lemma 5.6 and Lemma[5.7 one needs estimates for the non-linear part.

The lemma below is a simple consequence of (5.28) and Proposition 3.4

Lemma 5.13. Let $1 \leq q \leq p<\infty$.

(1) If $u, v \in M_{2 q}^{2 p}$, then $P \mathcal{Q}(u, v) \in N_{p, q, \infty}^{-1}$ with

$$
\|P \mathcal{Q}(u, v)\|_{N_{p, q, \infty}^{-1}} \leq C\|u\|_{M_{2 q}^{2 p}}\|v\|_{M_{2 q}^{2 p}} .
$$

(2) If $u, v \in \mathcal{M}_{2 q}^{2 p}$, then $P \mathcal{Q}(u, v) \in \mathcal{N}_{p, q, \infty}^{-1}$ with

$$
\|P \mathcal{Q}(u, v)\|_{\mathcal{N}_{p, q, \infty}^{-1}} \leq C\|u\|_{\mathcal{M}_{2 q}^{2 p}}\|v\|_{\mathcal{M}_{2 q}^{2 p}} .
$$

Again, it is necessary to introduce an auxiliary space. Given (5.31) and (5.32), there is a natural candidate.

Definition 5.14. Let $\mathcal{G}=\mathcal{G}_{T}$ be the space of measurable functions u on $(0, T) \times \mathbb{R}^{n}$ such that

$$
\|u\|_{\mathcal{G}}=\sup _{0<t \leq T} t^{1 / 2-n / 4 p}\|u(t, \cdot)\|_{M_{2 q}^{2 p}}<\infty .
$$

The norm in $\mathcal{G}$ reminds us of the artificial norm used by Kato [Ka1] in analyzing NS on the limit space $L^{n}$ :

$$
\|u\|_{*}=\sup _{t>0}\left\{t^{\alpha / 2}\|u(t)\|_{L^{q}}\right\}, \quad q>n, \alpha=1-n / q .
$$

Now, let $u_{j}$ be the Picard iterates and set $G_{j}=\left\|u_{j}\right\|_{\mathcal{G}}, F_{j}=\left\|v_{j}\right\|_{\mathcal{G}}$, with $v_{j}=u_{j}-u_{j-1}$, as before.

Lemmas similar to Lemma 5.9 and Lemma 5.10 hold in this case as a consequence of Lemmas 5.6, 5.7 and 5.13,

Lemma 5.15 ([KY] $)$. (1) Under the hypotheses of Theorem [5.12, there exists $C_{0}>$ 0 , independent of $a$, and a time $T \leq 1$ such that

$$
G_{0}<C_{0} \delta \text {. }
$$


(2) Moreover, there exists $C_{1}>0$ independent of $T$ such that, for all $j \geq 0$,

$$
G_{j+1} \leq G_{0}+C_{1} G_{j}^{2} .
$$

The lemma gives a uniform bound on $G_{j}$, namely $G_{j} \leq \omega(\delta), \forall j \geq 0$, with $\omega(\delta)=2 C_{0} \delta /\left(1+\sqrt{1-4 C_{0} C_{1} \delta}\right)$ the smaller root of $C_{1} x^{2}-x+C_{0} \delta=0$.

Lemma 5.16. There exists $C_{2}$ independent of $T \leq 1$ such that the inequality

$$
F_{j+1} \leq C_{2} \omega(\delta) F_{j}
$$

holds $\forall j \in \mathbb{N}$.

Now, choose $\delta$ so small that $\omega(\delta) \leq 1 / 2 C_{2}$. Then, $F_{j} \leq F_{j} / 2$ so that $\sum_{j} F_{j}<\infty$; that is, the sequence $\left\{u_{j}\right\}$ converges in $\mathcal{G}$ to a solution of (5.30), with $\|u\|_{\mathcal{G}} \leq$ $\omega(\delta)=K$, and Part (1) is proved. The rest of the theorem follows as Theorem 5.4 Uniqueness is established via a simple modification of Proposition 5.11.

Finally, we have global-in-time existence if a sufficiently small initial data $a \in$ $\mathcal{N}_{p, q, \infty}^{n / p-1}$ is selected parallel to the analysis of (5.5).

5.4. Extensions. As we discussed earlier, it is rather natural to expect (local) existence to hold in the subcritical range of indices for arbitrary initial data.

Recall that

$$
\begin{aligned}
& N_{p, q, \infty}^{s}=\left\{f \in \mathcal{S}^{\prime} \mid \sup _{0<t \leq 1}\left\{t^{-s / 2}\left\|e^{t \Delta} f\right\|_{M_{q}^{p}}<\infty\right\}\right\}, \\
& \mathcal{N}_{p, q, \infty}^{s}=\left\{f \in \mathcal{S}^{\prime} \mid \sup _{0<t}\left\{t^{-s / 2}\left\|e^{t \Delta} f\right\|_{\mathcal{M}_{q}^{p}}<\infty\right\}\right\},
\end{aligned}
$$

as long as $s<0$. In particular, if $a \in N_{p, q, r}^{s}$, automatically $u_{0}=e^{t \Delta} a \in \mathcal{A}$. Then, a simple modification of previous arguments gives the expected result.

Proposition 5.17. Let $f$ satisfy (5.6) and $\gamma, p, q, s$ be numbers such that

$$
\left\{\begin{array}{l}
\gamma \leq q \leq p, \\
n(\gamma-1)<2 p, \\
-2 / \gamma<s<0, \\
s>n / p-2 /(\gamma-1) .
\end{array}\right.
$$

Then, for all $a \in N_{p, q, \infty}^{s}$, there exists $T>0$ and a unique mild solution $u$ to (5.5) on $[0, T) \times \mathbb{R}_{x}^{n}$ that enjoys all the properties of Theorem 5.4

Proof. We prove existence and uniqueness only. Let $\mathcal{A}, A_{j}, B_{j}$ be as in the proof of Theorem 5.4. Show first that the sequence $\left\{A_{j}\right\}$ is bounded for sufficiently small $T$. By (5.38a), if $T \leq 1, A_{0} \leq\left\|u_{0}\right\|_{N_{p, q, \infty}^{s}}$. As in the proof of Lemma 5.9 ([KY], pp. 993-994), we obtain the following estimate:

$$
\left\|u_{j+1}(t)-u_{0}(t)\right\|_{M_{q}^{p}} \leq C_{1} t^{s / 2} t^{\epsilon}\left(A_{j}^{\gamma}+T^{-s \gamma / 2}\right),
$$

with $\epsilon=1+(\gamma-1)(s / 2-n / 2 p)$. Note that $\epsilon>0$, because $s>n / p-2 /(\gamma-1)$.

Now, choose $T$ so small that

$$
C_{1} T^{\epsilon}\left(A_{0}+T^{-s \gamma / 2}\right) \leq 1 / 2^{\gamma}
$$


and proceed by induction. Assume that $C_{1} T^{\epsilon} A_{j}^{\gamma-1} \leq 1 / 2 \forall j \leq k$. Then $A_{j+1} \leq$ $1 / 2 A_{j}+\nu$ with $\nu=T^{\epsilon-s \gamma / 2}$ small. In fact, $\epsilon-s \gamma / 2>0$, as $2 p>(\gamma-1) n$. Consequently,

$$
A_{j+1} \leq \frac{1}{2^{j+1}} A_{0}+\nu \sum_{0 \leq i \leq j} 2^{-i} \leq 2\left(A_{0}+\nu\right) .
$$

In particular, by means of estimate (5.41), for $j=k$,

$$
C_{1} T^{\epsilon} A_{k+1}^{\gamma-1} \leq C_{1} T^{\epsilon} 2^{\gamma-1}\left(A_{0}+\nu\right)^{\gamma-1} \leq \frac{1}{2^{\gamma}} 2^{\gamma-1}=\frac{1}{2},
$$

which proves the induction step and gives finally

$$
A_{k} \leq 2\left(A_{0}+\nu\right) \equiv K, \quad \forall k .
$$

We show next that the series $\sum_{j} B_{j}$ converges. This is enough to prove $u_{j} \rightarrow u$ in $\mathcal{A}$. Following the proof of Lemma $[5.10([\mathrm{KY}]$, pp. 995-996), we obtain the estimate:

$$
\left\|v_{j+1}\right\|_{M_{q}^{p}} \leq C_{2} t^{s / 2} t^{\epsilon} B_{j}\left(\left[2 A_{0}+2 \nu\right]^{\gamma-1}+T^{s(1-\gamma) / 2}\right),
$$

with $\epsilon$ and $\nu$ as before. Again, choose $T_{1} \leq T$ so small that

$$
C_{2} T_{1}^{\epsilon}\left(\left[2 A_{0}+2 \nu\right]^{\gamma-1}+T^{s(1-\gamma) / 2}\right) \leq \frac{1}{2} .
$$

Then, $B_{j+1} \leq 1 / 2 B_{j}$, that is, $\sum_{j} B_{j}<\infty$.

As far as uniqueness is concerned, we cannot directly quote Proposition 5.11 because $K$ may not be small for $T$ small. But here (5.20) is replaced by

$$
H\left(T^{\prime}\right) \leq C_{2}\left(T^{\prime}\right)^{\epsilon}\left(K^{\gamma-1}+T^{s(1-\gamma) / 2}\right) H\left(T^{\prime}\right) .
$$

Hence $H(T) \equiv 0$ for $T$ sufficiently small.

Remark 5.18. The constants $C_{1}$ and $C_{2}$, and thus the length of the existence time $T$, depend only on the size of the initial data. Therefore, the solution persists for as long as $\|u(t)\|_{N_{p, q, \infty}^{s}}$ stays finite.

A similar derivation, using instead Lemmas 5.15 and $[5.16$ yields the following conclusion for the Navier-Stokes equation.

Proposition 5.19. Let $p, q, s$ be numbers such that

$$
\left\{\begin{array}{l}
1 \leq q \leq p, \\
p>n, \\
n / p-1<s<0 .
\end{array}\right.
$$

Then, for all $a \in N_{p, q, \infty}^{s}$, there exists $T>0$ and a unique mild solution $u$ to (5.27) on $[0, T) \times \mathbb{R}_{x}^{n}$ that enjoys all the properties of Theorem 5.12.

Note that the condition $s<0$ is used in deriving (5.40), which requires $s(1-\gamma)>$ 0 , and here $\gamma>1$.

To improve this local existence result we turn to the theory of adapted spaces Can, which we briefly recall. These are functional Banach spaces $E$ (with translation-invariant norm) such that

$$
\left\|\psi_{j}(D)(f g)\right\|_{E} \leq \eta_{j}\|f\|_{E}\|g\|_{E}, \quad \forall j \in \mathbb{Z},
$$


where

$$
\sum_{j} 2^{-|j|} \eta_{j}<\infty .
$$

The two conditions above are sufficient (but clearly not necessary) to give a local Lipschitz condition on the map $\mathfrak{N}$, so that local-in-time existence follows. Intuitively, it is enough to bound the non-linear part of $\mathfrak{N}$. When frequencies are restricted to the shell $D_{j}$, the operator $e^{t \Delta} P$ has symbol comparable to $2^{j} \exp \left(-t 4^{j}\right)$. So, if $t>4^{j}$, the negative exponential will control the growth of $\sigma(P)$ and the quadratic part. If $t \leq 4^{j}$, the smoothing effect of the heat semigroup is small, but $\left\|P \psi_{j}(D)(u \otimes u)\right\|_{E}$ then contributes at most $4^{j}<t^{-1}$ (because of (5.48)), which gives integrability at $t=0$.

It can be shown that $L^{p}$ and Morrey spaces are adapted for $p \in(n, \infty)$ (cf. Proposition 5.3). Moreover, any functional Banach space that is also an algebra is adapted, because then (5.47) holds with $\eta_{j}$ constant for all $j$. Therefore, we immediately obtain that BM spaces are adapted for $s>n / p$, and for $s \geq n / p$ if also $r=1$. Para-differential calculus will give further estimates.

In fact, if $n / 2 p<s<n / p$, from 3.68b

$$
\begin{aligned}
\left\|\psi_{j}(D)(u v)\right\|_{N_{p, q, r}^{s}} & \leq C 2^{j(n / p-s)}\|u \cdot v\|_{N_{p, q, r}^{2 s-n / p}} \\
& \leq C 2^{j(n / p-s)}\|u\|_{N_{p, q, r}^{s}}\|v\|_{N_{p, q, r}^{s}}
\end{aligned}
$$

since $\psi_{j}(D)$ is $O\left(2^{j \ell}\right)$ in $O P S_{1,0}^{-\ell}$. Therefore,

$$
\eta_{j} \sim 2^{j(n / p-s)}
$$

and (5.48) holds, because $p>n$. If $s=n / p$, we must use (3.69) instead to conclude

$$
\eta_{j} \lesssim 2^{j \epsilon}, \quad \forall \epsilon>0
$$

which is still sufficient for (5.48).

We cannot expect to reach $s=0$ this way, at least for small values of $q$, because when $q<2, f g$ may not even be locally integrable. We assume, therefore, $q \geq 2$ and we look more carefully at the paraproduct decomposition. As shown in Can,

$$
\begin{aligned}
\psi_{j}(D)(f g)=\psi_{j}(D) f \phi_{j-2}(D) g & +\psi_{j}(D) g \phi_{j-2}(D) f \\
& +\psi_{j}(D)\left(\sum_{k \geq j} \psi_{k}(D) f \psi_{k}(D) g\right)
\end{aligned}
$$

where $\phi_{j}=\sum_{k \leq j} \psi_{k}$. Furthermore, since $\psi_{j} \psi_{k} \equiv 0$ for $|j-k|>1$, it is enough to bound the Morrey norm of each of the terms in (5.52).

We start by recalling that, if $f \in L_{\text {loc }}^{1}$, then

$$
f \in M_{1}^{p} \Leftrightarrow e^{t \Delta}|f| \leq C t^{-n / 2 p}
$$

for $0<t \leq 1$. Since $\phi_{j}(\xi)$ and the symbol of $e^{t \Delta}$ have a similar behavior if $t=4^{-j}$, it follows that

$$
\left\|\phi_{j}(D) f\right\|_{L^{\infty}} \leq C 2^{j n / p}\left\|\phi_{j}(D) f\right\|_{M_{1}^{p}}
$$


Hölder's inequality then gives the same estimate for $M_{q}^{p}$, and Lemma 3.16 allows us to conclude that

$$
\begin{aligned}
\left\|\phi_{j}(D) f \psi_{j}(D) g\right\|_{M_{q}^{p}} & \leq\left\|\phi_{j}(D) f\right\|_{L^{\infty}}\left\|\psi_{j}(D) g\right\|_{M_{q}^{p}} \\
& \leq C 2^{j n / p} 2^{-2 j s}\|f\|_{N_{p, q, r}^{s}}\|g\|_{N_{p, q, r}^{s}},
\end{aligned}
$$

if also $s>0$.

As for the third term in (5.52), for notational convenience we set $\psi_{k}(D) f=f_{k}$, $\psi_{k}(D) g=g_{k}$. We observe first that

$$
\left\|f_{k} g_{k}\right\|_{M_{1}^{p / 2}} \leq\left\|f_{k}\right\|_{M_{q}^{p}}\left\|g_{k}\right\|_{M_{q^{\prime}}^{p}} \leq\left\|f_{k}\right\|_{M_{q}^{p}}\left\|g_{k}\right\|_{M_{q}^{p}}
$$

where $q^{\prime}$ is the conjugate exponent of $q$ and $q \geq q^{\prime}$, since $q \geq 2$.

While it is not true, in general, that $p(D): M_{1}^{p / 2} \rightarrow M_{q}^{p}$, if $p \in S_{1,0}^{-n / p}$ [Tay3, $\psi_{j}(D)$ does map $M_{1}^{p / 2}$ into $M_{q}^{p}$. However, proceeding this way yields an estimate for $\eta_{j}$ that is not sharp (cf. (5.54) $)$. Instead an elementary calculation shows that

$$
\|h\|_{M_{q}^{p}} \leq\|h\|_{L^{\infty}}^{1 / q^{\prime}}\|h\|_{M_{1}^{p / q}}^{1 / q} .
$$

Here, $h=\psi_{j}(D)\left(f_{k} g_{k}\right)$. Hence we use (5.54) again for the first piece with $p / q$ in place of $p$ :

$$
\left\|\psi_{j}(D)\left(f_{k} g_{k}\right)\right\|_{M_{q}^{p}} \leq C\left(2^{j n q / p}\right)^{1 / q^{\prime}}\left\|\psi_{j}(D)\left(f_{k} g_{k}\right)\right\|_{M_{1}^{p / q}}^{1 / q^{\prime}+1 / q} .
$$

We then need an estimate of the norm in $M_{1}^{p / q}$ in terms of $M_{1}^{p / 2}$ for functions of the form $\psi_{j}(D) u$. Because $\psi_{j}$ is supported on $|\xi| \sim 2^{j}$, we can restrict $t$ in (5.53) to $0<t<4^{-j}$, so that

$$
\psi_{j}(D) u \in M_{1}^{p / 2} \Rightarrow e^{t \Delta}\left|\psi_{j}(D) u\right| \leq C t^{n q / 2 p} 2^{j n(q / p-2 / p)},
$$

as $q / p-2 / p \geq 0$, which is equivalent to

$$
\left\|\psi_{j}(D) u\right\|_{M_{1}^{p / q}} \leq C 2^{j n(2 / p-q / p)}\left\|\psi_{j}(D) u\right\|_{M_{1}^{p / 2}} .
$$

Combining (5.56), (5.57), and (5.58), gives

$$
\begin{aligned}
\left\|\psi_{j}(D)\left(f_{k} g_{k}\right)\right\|_{M_{q}^{p}} & \leq C 2^{j n(q-1) / p} 2^{j n(2 / p-q / p)}\left\|f_{k} g_{k}\right\|_{M_{1}^{p / 2}} \\
& \leq C 2^{j n / p}\left\|f_{k}\right\|_{M_{q}^{p}}\left\|g_{k}\right\|_{M_{q}^{p}} .
\end{aligned}
$$

Finally, if $s>0$, similarly to (5.55),

$$
\left\|\psi_{j}(D)\left(\sum_{k \geq j} f_{k} g_{k}\right)\right\|_{M_{q}^{p}} \leq C\left(\sum_{k \geq 0} 2^{-2 s k}\right) 2^{j n / p} 2^{-2 j s}\|f\|_{N_{p, q, r}^{s}}\|g\|_{N_{p, q, r}^{s}} .
$$

Therefore, as before,

$$
\eta_{j} \sim 2^{j(n / p-s)} .
$$

We gather these results in the following corollary.

Corollary 5.20. The BM space $N_{p, q, r}^{s}$ is adapted to the Navier-Stokes equation for

(1) $s>n / 2 p$, if $p>n, 1 \leq q<2, r \in[1, \infty]$,

(2) $s>0$, if $p>n, 2 \leq q \leq p<\infty, r \in[1, \infty]$. 
In view of the Sobolev-type embedding of Theorem 2.4 the solution given by the previous corollary will behave like a classical solution for large $s$, very much in the spirit of Sobolev spaces.

Let us summarize our extensions so far. For the inhomogeneous BM space $N_{p, q, \infty}^{s}$, we have local-in-time existence and uniqueness if

$$
n / p-1<s<0 \text {, or } s>n / 2 p,
$$

for arbitrary initial data. If $s=n / p-1$, existence and uniqueness hold for data satisfying (5.29).

If $s=n / p-1$, existence is global in time in the homogeneous BM space $\mathcal{N}_{p, q, \infty}^{n / p-1}$ for sufficiently small initial data.

We conclude this section by comparing the previous results with those of Karch. In $[\mathrm{Kr}]$, he considered an IVP with data in $B E^{\alpha}$ for a similar class of parabolic equations; namely,

$$
\left\{\begin{array}{l}
u_{t}=\Delta u+B(u, u), \\
u(0)=a .
\end{array}\right.
$$

Here, $B$ is a bilinear form with scaling order $b<2$.

Definition 5.21. $B$ is said to have scaling order equal to $b \in \mathbb{R}$ if

$$
B\left(u_{\lambda}, v_{\lambda}\right)=\lambda^{b}(B(u, v))_{\lambda},
$$

where $u_{\lambda}(x)=u(\lambda x)$.

Note that the IVP (5.5) is of the form (5.60) (with $b=0$ ) only if $f$ is homogeneous of degree 2 (so, $\gamma=2$ ), while NS becomes (5.60) for $b=1$. Recall, once again, that $B E_{p}^{\alpha}$ can be identified with $\mathcal{N}_{p, q, \infty}^{s}$ if $E_{p}=\mathcal{M}_{q}^{p}$ and $\alpha=-s, s<0$. Then the existence criteria established in Section [5.2 (Theorem 1 in [KY]) and in Section 5.3 (Theorem 3 in $[\mathrm{KY}]$ ) become exactly the content of Proposition 5.1 and Theorem $5.1 \mathrm{in}[\mathrm{Kr}$.

At the same time, other results in $[\mathrm{Kr}]$ can now be applied to the BM spaces, for example persistence and regularity of solutions. We just mention the following asymptotic condition.

Theorem $5.22([\mathrm{Kr}])$. Let $u_{1}$ and $u_{2}$ be two (global) mild solutions to the NavierStokes equation (5.27) with sufficiently small initial data $a_{1}, a_{2} \in \mathcal{N}_{p, q, \infty}^{n / p-1}$ respectively. If

$$
\lim _{t \rightarrow+\infty} t^{1 / 2-n / 4 p}\left\|e^{t \Delta}\left(a_{1}-a_{2}\right)\right\|_{\mathcal{M}_{q}^{p}}=0
$$

then

$$
\lim _{t \rightarrow+\infty} t^{1 / 2-n / 4 p}\left\|u_{1}(t)-u_{2}(t)\right\|_{\mathcal{M}_{q}^{p}}=0 .
$$

Condition (5.61) is satisfied, for example, if $a_{j} \in \mathcal{N}_{p, q, \infty}^{n / p-1} \cap \mathcal{N}_{p, q, \infty}^{n / p-1-\epsilon}, \epsilon>0$, $j=1,2$, given again the identification (5.38).

5.5. Analysis on compact manifolds. Let us consider again the Navier-Stokes and semi-linear heat equations now with $u(t, x)$ a function on $[0,+\infty) \times M$, and $a \in N_{p, q, \infty}^{s}(M)$. As usual, $M$ stands for a smooth, compact, Riemannian manifold without boundary. 
The analysis here is complicated by curvature terms and the non-local nature of the equation we consider. On the other hand, we do not have to deal with the behavior at infinity. Therefore, we will extend only the local existence results.

We start by considering again semi-linear heat equations. Since we cannot exploit finite propagation speed and work in local coordinates, even for small $t$, we will need to use the intrinsic characterization of BM spaces obtained in Section 4.2.

The idea is to "mimic" the proof of Theorem 5.4 on Euclidean space by replacing $\psi_{j}(D)$ with $\psi_{j}(\sqrt{-\Delta}), \Delta$ the Laplace operator on $M$.

Theorem 5.23. Suppose that $f$ satisfies (5.6) and assume $p, q, s$ are numbers such that

$$
\left\{\begin{array}{l}
\gamma \leq q \leq p \\
n(\gamma-1)<2 p \\
-2 / \gamma<s<0 \\
s \geq n / p-2 /(\gamma-1) .
\end{array}\right.
$$

Then, there exist positive constants $\delta, K$, such that, for every $a \in N_{p, q, \infty}^{s}(M)$ satisfying

$$
\limsup _{j \rightarrow \infty} 2^{j s}\left\|\psi_{j}(\sqrt{-\Delta}) a\right\|_{M_{q}^{p}(M)}<\delta,
$$

there are $T>0$ and a unique mild solution $u(t, x)$ to 5.5 on $[0, T) \times M$ such that

$$
\sup _{0<t \leq T} t^{-s / 2}\|u(t, \cdot)\|_{M_{q}^{p}(M)} \leq K \text {. }
$$

It is clearly enough to prove appropriate variants of Lemmas 5.6] through 5.10] Estimate (5.9a) follows here from the asymptotic expansion for the heat parametrix (Vol. 2 of [Tay1]) (cf. (4.25) and the pseudo-differential calculus of Section 3). As a matter of fact, for $\ell \geq 0, t^{\ell / 2} \sigma\left(e^{t \Delta}\right)$ is uniformly bounded in $S_{1,0}^{-\ell}$, so that for $\ell \geq s$, $1<q \leq p<\infty, r \in[1, \infty]$,

$$
\left\|e^{t \Delta} u\right\|_{N_{p, q, r}^{\ell}} \leq C t^{(s-\ell) / 2}\|u\|_{N_{p, q, r}^{s}},
$$

if $0<t \leq 1$. Estimate (5.10b) is then a consequence of (5.66) and the interpolation result

$$
\left(N_{p, q, r_{1}}^{s_{1}}(M), N_{p, q, r_{2}}^{s_{2}}(M)\right)_{\theta, r}=N_{p, q, r}^{s}(M), \quad(1-\theta) s_{1}+\theta s_{2}=s,
$$

which, in turn, is easily obtained from (4.53) exactly as in the Euclidean case (cf. KY, pp. 986-987).

Next, we prove a version of Lemma 5.7 .

Lemma 5.24. Let $1<q \leq p<\infty$ and $s<\ell$. There exists a positive $A=$ $A(p, q, s, \ell)$ such that, for all $u \in N_{p, q, \infty}^{s}$ and $B$ satisfying

$$
A \limsup _{j \rightarrow \infty} 2^{s j}\left\|\psi_{j}(\sqrt{-\Delta}) u\right\|_{M_{q}^{p}}<B,
$$

there is a $T>0$ such that

$$
\sup _{0<t \leq T} t^{(\ell-s) / 2}\left\|e^{t \Delta} u\right\|_{N_{p, q, 1}^{\ell}}<B .
$$


Proof. The proof is similar to that of Lemma [5.7, but agair 15 we have to treat an infinite asymptotic expansion for the symbol of $\psi_{j}(\sqrt{-\Delta})$.

By the analogue of (5.10b) obtained above, there exists $C_{0}$ such that

$$
\left\|e^{t \Delta} u\right\|_{N_{p, q, 1}^{\ell}} \leq C_{0} t^{(s-\ell) / 2}\|u\|_{N_{p, q, \infty}^{s}},
$$

with $C_{0}$ independent of $T, 0<T \leq 1$. Also, from (5.68),

$$
2^{j s}\left\|\psi_{j}(\sqrt{-\Delta}) u\right\|_{M_{q}^{p}}<\alpha=B / A, \quad \forall j \geq m,
$$

for some fixed $m \in \mathbb{N}$.

Let $\Phi_{m}(\sqrt{-\Delta}) u=\psi_{0}\left(2^{-m} \sqrt{-\Delta}\right) u=u_{1}$ and set $u_{2}=u-u_{1}$. As discussed in Section 4.2, there is an asymptotic expansion for the symbol of $\Phi_{m}(\sqrt{-\Delta})$ :

$$
\sigma\left(\Phi_{m}(\sqrt{-\Delta})\right) \sim \sum_{k \geq 0} 4^{-k m} \psi_{0}^{(2 k)}\left(2^{-m}|\xi|_{x}\right) p_{k}\left(x, 2^{-m} \xi\right),
$$

with $p_{k}$ a polynomial of degree $k$ and $|\xi|_{x}=\left(\sum_{i, j} g^{i j}(x) \xi_{i} \xi_{j}\right)^{1 / 2}$. Next, note that

$$
\begin{aligned}
& \psi_{0}\left(2^{-m}|\xi|_{x}\right) \psi_{j}\left(|\xi|_{x}\right) \\
& \quad= \begin{cases}\psi_{j}\left(|\xi|_{x}\right) & j<m-N, \\
\sim \sum_{l=m-N}^{m+N} \psi_{l}\left(|\xi|_{x}\right) \psi_{j}\left(|\xi|_{x}\right) & m-N \leq j \leq m+N, \\
0 & j>m+N\end{cases}
\end{aligned}
$$

and

$$
\begin{aligned}
& \left(1-\psi_{0}\left(2^{-m}|\xi|_{x}\right)\right) \psi_{j}\left(|\xi|_{x}\right) \\
& \quad= \begin{cases}0 & j<m-N, \\
\sim \sum_{l=m-N}^{m+N} \psi_{l}\left(|\xi|_{x}\right) \psi_{j}\left(|\xi|_{x}\right) & m-N \leq j \leq m+N, \\
\psi_{j}\left(|\xi|_{x}\right) & j<m+N\end{cases}
\end{aligned}
$$

where $N$ is independent of $j$ and $x$, with similar estimates for $\psi_{0}^{(2 k)}\left(2^{-m}|\xi|_{x}\right)$. In particular, we can write

$$
\begin{aligned}
& \psi_{j}(\sqrt{-\Delta}) \Phi_{m}(\sqrt{-\Delta})=R_{j}^{\infty}, \quad \text { if } j>m-N, \\
& \psi_{j}(\sqrt{-\Delta})\left(\operatorname{Id}-\Phi_{m}(\sqrt{-\Delta})\right)=\tilde{R}_{j}^{\infty}, \quad \text { if } j<m+N,
\end{aligned}
$$

with the symbol of $R_{j}^{\infty}$ and $\tilde{R}_{j}^{\infty}$ rapidly decreasing in both $\xi$ and $j$. Consequently,

$$
\begin{aligned}
& \left\|u_{1}\right\|_{N_{p, q, \infty}^{s}} \leq C_{1} \sup _{j \leq m+N}\left\{2^{j s}\left\|\psi_{j}(\sqrt{-\Delta}) \Phi_{m}(\sqrt{-\Delta}) u\right\|_{M_{q}^{p}}\right\}, \\
& \left\|u_{2}\right\|_{N_{p, q, \infty}^{s}} \leq C_{2} \sup _{j \geq m-N}\left\{2^{j s}\left\|\psi_{j}(\sqrt{-\Delta})\left(\operatorname{Id}-\Phi_{m}(\sqrt{-\Delta})\right) u\right\|_{M_{q}^{p}}\right\},
\end{aligned}
$$

for some positive constants $C_{j}, j=1,2$. Moreover, because of (5.73a) and 5.73b), these constants depend only on $\left\|\check{\psi}_{j}\right\|_{L^{1}}$.

It follows from (5.71) that

$$
\left\|u_{2}\right\|_{N_{p, q, \infty}^{s}} \leq K \sup _{j \geq m-N}\left\{2^{j s}\left\|\psi_{j}(\sqrt{-\Delta}) u\right\|_{M_{q}^{p}}\right\} \leq K \alpha,
$$

\footnotetext{
${ }^{15}$ Cf. Section 4.2
} 
with $K$ depending only on $\left\{\psi_{j}\right\}$. Choose $A=C_{0} K$. Then, for $0<t \leq 1$,

$$
\begin{aligned}
t^{(\ell-s) / 2}\left\|e^{t \Delta} u_{2}\right\|_{N_{p, q, 1}^{\ell}} & \leq C_{0}\left\|u_{2}\right\|_{N_{p, q, \infty}^{s}} \\
& \leq C_{0} K \alpha \\
& <\frac{A \alpha+B}{2} .
\end{aligned}
$$

As for $u_{1}, u_{1} \in N_{p, q, \infty}^{s}$ for every $s$, because $\Phi_{m}(\sqrt{-\Delta})$ is a smoothing operator. Consequently,

$$
\begin{aligned}
t^{(\ell-s) / 2}\left\|e^{t \Delta} u_{1}\right\|_{N_{p, q, 1}^{\ell}} & \leq C_{0} T^{(\ell-s) / 4}\left\|u_{1}\right\|_{N_{p, q, \infty}^{(s+\ell) / 2}} \\
& <\frac{B-\epsilon}{2},
\end{aligned}
$$

with $0<\epsilon<B$ for $T$ sufficiently small. If $\epsilon$ is chosen equal to $A \alpha$, (5.77) and (5.78) combined give the desired estimate.

The analogues of Lemma 5.9] and Lemma 5.10 are now established exactly as in the Euclidean case. Moreover, using once more Definition 4.5] and Proposition 2.26] we have for $s<0$,

$$
u \in N_{p, q, \infty}^{s}(M) \Leftrightarrow \sup _{0<t \leq 1} t^{-s / 2}\left\|e^{t \Delta} u\right\|_{M_{q}^{p}(M)}<\infty .
$$

Therefore, one obtains again local existence for arbitrary data in the subcritical range.

Proposition 5.25. Let $f$ satisfy (5.6) with $\gamma>1$, and let $\gamma, p, q, s$ be numbers such that

$$
\left\{\begin{array}{l}
\gamma \leq q \leq p, \\
n(\gamma-1)<2 p, \\
-2 / \gamma<s<0, \\
s>n / p-2 /(\gamma-1) .
\end{array}\right.
$$

Then, for all $a \in N_{p, q, \infty}^{s}(M)$, there exists $T>0$ and a unique mild solution $u$ to (5.5) on $[0, T) \times M$.

We now turn to the Navier-Stokes system. As we data mentioned at the beginning of Section 5, curvature adds an extra term to the momentum equation in (5.26). The "correct" version of the Navier-Stokes equations on a curved manifold is the following system [EM]:

$$
\left\{\begin{array}{l}
\partial_{t} u(t, x)=\Delta u(t, x)+2 \mathbb{P} \operatorname{Ric} u(t, x)-\mathbb{P} \operatorname{div}(u \otimes u)(t, x), \\
\operatorname{div} u(t, x)=0, \\
u(0, x)=a(x),
\end{array}\right.
$$

where $\Delta$ is the Laplace-Beltrami operator and $\operatorname{Ric}^{j k}=R_{i}{ }^{j i k}$ is the Ricci tensor on $M$, i.e., the contraction of the curvature tensor $R^{i j k l}$.

Without going into detail (see Tay3 and the discussion there), we just say that the Laplacian enters the momentum equation by applying the divergence theorem to the stress or deformation tensor $S$, which represents the forces exerted on some volume of fluid across a unit surface element. So, one should actually have $\operatorname{div} S$ in place of the Laplacian in the equation. But on $\mathbb{R}^{n} \operatorname{div} S u=\Delta u$, while on a manifold $\operatorname{div} S u=\Delta u+2 \operatorname{Ric} u$, if $\operatorname{div} u=0$. 
Here, we "mimic" the proof of Theorem [5.12] The term $\mathbb{P}$ Ric $u$ does not substantially modify the proof, because it is an operator of order 0 .

Proposition 5.26. Let $1<q \leq p<\infty$ and $p>n$. Then, there exist $\delta, K>0$ such that for every $a \in N_{p, q, \infty}^{n / p-1}(M)$ satisfying

$$
\left\{\begin{array}{l}
\lim \sup _{j \rightarrow \infty} 2^{j(n / p-1)}\left\|\psi_{j}(\sqrt{-\Delta}) a\right\|_{M_{q}^{p}(M)}<\delta, \\
\operatorname{div} a(x)=0,
\end{array}\right.
$$

there are $T>0$ and a mild solution $u(t, x)$ of (5.81) on $[0, T) \times \mathbb{R}^{n}$ such that

$$
\sup _{0<t \leq T} t^{1 / 2-n / 4 p}\|u(t, \cdot)\|_{M_{2 q}^{2 p}(M)} \leq K .
$$

Given all previous results, it is enough to obtain an analogue of Lemma 5.13

Lemma 5.27. Let $1<q \leq p<\infty$. If $u \in M_{2 q}^{2 p}(M)$, then $\mathbb{P}(\operatorname{div}(u \otimes u)-2 \operatorname{Ric} u) \in$ $N_{p, q, \infty}^{-1}$ with

$$
\|\mathbb{P}(\operatorname{div}(u \otimes u)-2 \operatorname{Ric} u)\|_{N_{p, q, \infty}^{-1}} \leq C\left[\|u\|_{M_{2 q}^{2 p}}^{2}+\|u\|_{M_{2 q}^{2 p}}\right] .
$$

The lemma is an immediate consequence of the results in Section 3 Since $\mathbb{P}$ Ric $\in$ $O P \Sigma_{1}^{0}, \mathbb{P}$ Ric $: N_{p, q, r}^{s}(M) \rightarrow N_{p, q, r}^{s}(M), \forall s$, and

$$
\begin{aligned}
\|\mathbb{P}(\operatorname{div}(u \otimes u)-2 \operatorname{Ric} u)\|_{N_{p, q}^{-1}, \infty} & \leq C\left[\|u \otimes u\|_{M_{q}^{p}}+\|u\|_{M_{q}^{p}}\right] \\
& \leq C\left[\|u\|_{M_{2 q}^{2 p}}^{2}+\|u\|_{M_{2 q}^{2 p}}\right],
\end{aligned}
$$

because of the inclusions

$$
M_{2 q}^{2 p} \subset M_{q}^{p} \subset N_{p, q, \infty}^{0} .
$$

Finally, we mention that the theory of adapted spaces can also be developed for compact (boundaryless) manifolds. It is again sufficient to replace (5.47) by the following condition:

$$
\left\|\psi_{j}(\sqrt{-\Delta}) f g\right\|_{E} \leq \eta_{j}\|f\|_{E}\|g\|_{E},
$$

where $\eta_{j}$ still satisfies (5.48).

To prove that the map $\mathfrak{N}$ is a contraction for short-time, we utilize the asymptotic expansion for the heat parametrix in local coordinates (see [Tay1, Vol. 2), and arguments similar to those leading to Lemma 5.24

\section{ACKNOWLEDGEMENTS}

The author thanks her Ph.D. adviser, Michael E. Taylor, for his continuous support and encouragement, and Richard Beals, Roberto Camassa, Peter Jones, Norberto Kerzman, and Mark Williams for helpful discussions.

\section{REFERENCES}

[BC] H. Bahouri, J.- Y. Chemin, Équations de transport relatives à des champs des vecteurs non-lipschitziens et mécanique des fluides, Arch. Rational Mech. Anal. 127 (1994) no. 2, 159-181. MR 95g:35164

[BL] J. Bergh, J. Löfström, Interpolation Spaces. An introduction, Grundlehren der Mathematischen Wissenschaften 223, Springer-Verlag, Berlin-New York, 1976. MR 58:2349

[BRV] O. Blasco, A. Ruiz, L. Vega, Non-interpolation in Morrey-Campanato and block spaces, Ann. Scuola Norm. Sup. Pisa Cl. Sci. (4) 28 (1999) no. 1, 31-40. MR 2000c:46048 
[Bo] J.-M. Bony, Calcul symbolique et propagation des singularités pour les équations aux dérivées partielles non linéaires, Ann. Sci. École. Norm. Sup. (4) 14 (1981) no. 2, 209246. MR 84h:35177

[Bour] G. Bourdaud, Une algèbre maximale d'opérateurs pseudo-différentiels, Comm. Partial Differential Equations 13 (1988) no. 9, 1059-1083. MR 89g:47063

[Bour2] _ Analyse fonctionnelle dans l'espace euclidien, Publications Mathématiques de l'Université Paris VII 23, Université de Paris VII, U.E.R. de Mathématiques, Paris, 1986. MR 89a:46001

[Can] M. Cannone, Ondelettes, paraproduits et Navier-Stokes, Diderot Editeur, Arts et Sciences, Paris, 1995. MR 2000e:35173

[CX] Z. M. Chen, Z. Xin, Homogeneity criterion for the Navier-Stokes equations in the whole spaces, J. Math. Fluid Mech. 3 (2001), no. 2, 152-182. MR 2002d:76033

$[\mathrm{ChM}]$ A. J. Chorin, J. E. Marsden, A mathematical introduction to fluid mechanics, Texts in Applied Mathematics 4, Springer-Verlag, New York, 1990. MR 91e:76002

[CM] R. R. Coifman, Y. Meyer, Au delà des opérateurs pseudo-différentiels, Astérisque 57, Société Mathématique de France, Paris, 1978. MR 81b:47061

[Dn] R. Danchin, Existence globale dans des espaces critiques pour le système de NavierStokes compressible, Seminaire: Équations aux Dérivées Partielles, 1998-1999, Exp. no. XXI, École Polytech., Palaiseau, 1999.

[EM] D. G. Ebin, J. Marsden, Groups of diffeomorphisms and the notion of an incompressible fluid, Ann. of Math. 92 (1970) no. 2, 102-163. MR 42:6865

[Fe] P. Federbush, Navier and Stokes meet the wavelet, Comm. Math. Phys. 155 (1993) no. 2, 219-248. MR 94g:35171

[FJ1] M. Frazier, B. Jawerth, Decomposition of Besov Spaces, Indiana University Math. J., 34 (1985) no. 4, 777-799. MR 87h:46083

[FJ2] , A discrete transform and decompositions of distribution spaces, J. Funct. Anal. 93 (1990), no. 1, 34-170. MR 92a:46042

[FK] H. Fujita, T. Kato, On the Navier-Stokes initial value problem. I, Arch. Rational Mech. Anal. 16 (1964), 269-315. MR 29:3774

[GM] Y. Giga, T. Miyakawa, Navier-Stokes flow in $\mathbb{R}^{3}$ with measures as initial vorticity and Morrey spaces, Comm. Partial Differential Equations 14 (1989) no. 5, 577-618. MR 90e:35130

[GMO] Y. Giga, T. Miyakawa, H. Osada, Two-dimensional Navier-Stokes flow with measures as initial vorticity, Arch. Rational Mech. Anal. 104 (1988) no. 3, 223-250. MR 90i:35217

[Ho] L. Hörmander, Pseudo-differential operators and hypoelliptic equations. Singular Integrals (Proc. Sympos. Pure Math., Vol. X, Chicago, Ill., 1966), 138-183, Amer. Math. Soc., Providence, R.I., 1967. MR 52:4033

[JN] F. John, L. Nirenberg, On Functions of Bounded Mean Oscillation, Comm. Pure Appl. Math., 14 (1961), 415-426. MR 24:A1348

[Kr] G. Karch, Scaling in nonlinear parabolic equations, J. Math. Anal. Appl. 234 (1999) no. 2, 534-558. MR 2000h:35068

[Ka1] T. Kato, Strong $L^{p}$-solutions of the Navier-Stokes equation in $\mathbb{R}^{m}$, with applications to weak solutions, Math. Zeit. 187 (1984) no. 4, 471-480. MR 86b:35171

[Ka2] , Strong solutions of the Navier-Stokes equation in Morrey spaces, Boll. Soc. Brasil. Mat. (N.S.) 22 (1992) no. 2, 127-155. MR 93i:35104

[Ka3] The Navier-Stokes equation for an incompressible fluid in $\mathbb{R}^{2}$ with a measure as the initial vorticity, Differential Integral Equations 7 (1994) no. 3-4, 949-966. MR 95b:35173

[KoT] H. Koch, D. Tataru,Well-posedness for the Navier-Stokes equations, Adv. Math. 157 (2001), no. 1, 22-35. MR 2001m:35257

[KY] H. Kozono, M. Yamazaki, Semilinear heat equations and the Navier-Stokes equation with distributions in new function spaces as initial data, Comm. Partial Differential Equations 19 (1994) no. 5-6, 959-1014. MR 95d:35077

[KY2] - The Navier-Stokes equation with distributions as initial data and application to self-similar solutions. New trends in microlocal analysis (Tokyo, 1995), 125-141, Springer, Tokyo, 1997. MR 2000b:35200

[LMR] P.G. Lemarié-Rieusset, Some remarks on the Navier-Stokes equations in $\mathbb{R}^{3}$, J. Math. Phys. 39 (1998), n. 8, 4108-4118. MR 99f:35163 
[Lr] J. Leray, Etude de diverses équations intégrales nonlinéaires et de quelques problèmes que pose l'hydrodynamique, J. Math. Pures Appl., 12 (1933), 1-82.

[Lio] P.- L. Lions, Mathematical topics in fluid mechanics. Vol. 1. Incompressible models. Oxford Lecture Series in Mathematics and its Applications 3, Oxford Science Publications, The Clarendon Press, Oxford University Press, New York, 1996. MR 98b:76001

[MP] C. Marchioro, M. Pulvirenti, Mathematical theory of incompressible nonviscous fluids, Applied Mathematical Sciences 96, Springer-Verlag, New York, 1994. MR 94k:76001

[Ma] J. Marschall, Pseudodifferential operators with coefficients in Sobolev spaces, Trans. Amer. Math. Soc. 307 (1988) no. 1, 335-361. MR 89k:35262

[Maz1] A.L. Mazzucato, Analysis of the Navier-Stokes and Other Non-Linear Evolution Equations with Initial Data in Besov-Type Spaces, Ph.D. Thesis, University of North Carolina, Chapel Hill, NC, 2000.

[Maz2] Decomposition of Besov-Morrey spaces, to appear in the Proceedings of the Conference on Harmonic Analysis, AMS Series in Contemporary Mathematics.

[Me] Y. Meyer, Régularité des solutions des équations aux dérivées partielles non linéaires (d'après J.- M. Bony), Sem. Bourbaki, Vol. 1979/80, 293-302, Lectures Notes in Math. 842, Springer, Berlin-New York, 1981. MR 84c:35015

[Miy] T. Miyakawa, On Morrey spaces of measures: basic properties and potential estimates, Hiroshima Math. J., 20 (1990), 213-222. MR 91k:46021

$[\mathrm{Pa}]$ A. Pazy, Semigroups of linear operators and applications to partial differential equations, Applied Mathematical Sciences 44, Springer-Verlag, New York-Berlin, 1983. MR 85g:47061

[Pe] J. Peetre, On the theory of $\mathcal{L}_{p, \lambda}$ spaces, J. Functional Analysis 4 (1969), 71-87. MR 39:3300

[Ru1] T. Runst, Paradifferential operators in spaces of Triebel-Lizorkin and Besov type, Z. Anal. Anwendungen 4 (1985) no. 6, 557-573. MR 87c:35038

[Ru2] T. Runst, Pseudodifferential operators of the "exotic" class $L_{1,1}^{0}$ in spaces of Besov and Triebel-Lizorkin type, Ann. Global Anal. Geom. 3 (1985) no. 1, 13-28. MR 87d:47065

[SaT] M. Sable-Tougeron, Régularité microlocale pour des problèmes aux limites non linéaires, Ann. Inst. Fourier (Grenoble) 36 (1986) no. 1, 39-82. MR 88b:35021

[ST] R. Schrader, M. E. Taylor, Semiclassical asymptotics, gauge fields, and quantum chaos, J. Functional Analysis 83 (1989) no. 2, 258-316. MR 90i:58198

[SeSo] A. Seeger, C. D. Sogge, On the boundedness of functions of (pseudo-) differential operators on compact manifolds, Duke Math. J. 59 (1989) no. 3, 709-736. MR 91d:58244

[SiT] W. Sickel, H. Triebel, Hölder inequalities and sharp embeddings in function spaces of $B_{p q}^{s}$ and $F_{p q}^{s}$ type, Z. Anal. Anwendungen 14 (1995) no. 1, 105-140. MR 96h:46062

[Tat] D. Tataru, Local and global results for wave maps. I., Comm. Partial Differential Equations 23 (1998), no. 9-10, 1781-1793. MR 99j:58209

[Tay1] M. E. Taylor, Partial Differential Equations, Applied Mathematical Sciences 115, 116, 117, Springer-Verlag, New York, 1996. MR 98b:35002b MR 98b:35003 MR 98k:35001

[Tay2] , Pseudodifferential operators and nonlinear PDE, Progress in Mathematics 100, Birkhäuser, Boston, MA, 1991. MR 92j:35193

[Tay3] _ Analysis on Morrey Spaces and applications to Navier-Stokes and other evolution equations, Comm. Partial Differential Equations 17 (1992) no. 9-10, 1407-1456. MR 94b:35218

[Tay4] , Pseudodifferential Operators, Princeton Mathematical Series 34, Princeton University Press, Princeton, N.J., 1981. MR 82i:35172

[Tay5] , Noncommutative harmonic analysis, Mathematical Surveys and Monographs 22, American Mathematical Society, Providence, R.I., 1986. MR 88a:22021

[Tay6] Microlocal analysis on Morrey spaces, Singularities and oscillations (Minneapolis, MN, 1994/1995), 97-135, IMA Vol. Math. Appl., 91, Springer, New York, 1997. MR 99a:35010

[Tem] R. Temam, Navier-Stokes equations. Theory and numerical analysis, Studies in Mathematics and its Applications 2, North-Holland, Amsterdam-New York-Oxford, 1977. MR 58:29439

[To] R. Torres, Continuity properties of pseudodifferential operators of type 1,1, Comm. Partial Differential Equations 15 (1990) no. 9, 1313-1328. MR 91m:47079 
[Tr] F. Treves, Introduction to pseudodifferential and Fourier integral operators, The University Series in Mathematics, Plenum Press, New York-London, 1980. MR 82i:35173]

[Trieb1] H. Triebel, Interpolation theory, function spaces, differential operators, North-Holland Mathematical Library 18, North-Holland, Amsterdam-New York, 1978. MR 80i:46032b

[Trieb2] H. Triebel, Theory of function spaces, Monographs in Mathematics 78, BirkhäuserVerlag, Basel-Boston, 1983. MR 86j:46026

[Trieb3] — Theory of function spaces. II, Monographs in Mathematics 84, BirkhäuserVerlag, Basel, 1992. MR 93f:46029

[Uch] A. Uchiyama, A constructive proof of the Fefferman-Stein decomposition of $B M O\left(\mathbb{R}^{n}\right)$, Acta Math. 148 (1982), 215-241. MR 84h:42037

[Vi1] M. Vishik, Hydrodynamics in Besov spaces, Arch. Rational Mech. Anal. 145 (1998) no. 3, 197-214. MR 2000a:35201

[Vi2] Incompressible flows of an ideal fluid with vorticity in borderline spaces of Besov type, Ann. Sci. École Norm. Sup. (4) 32 (1999) no. 6, 769-812. MR 2000i:76008

[We] F. B. Weissler, Local existence and nonexistence for semilinear parabolic equations in $L^{p}$, Indiana Univ. Math. J. 29 (1980) no. 1, 79-102. MR 81c:35072

$[\mathrm{Wu}] \mathrm{J} . \mathrm{Wu}$, Theory and applications of partial functional-differential equations, Applied Mathematical Sciences 119, Springer-Verlag, New York, 1996. MR 98a:35135

[Ya] M. Yamazaki, The Navier-Stokes equation in various function spaces, Amer. Math. Soc. Transl. 204 (2001), no. 2, 111-132.

Department of Mathematics, Yale University, P.O. Box 208283, 10 Hillhouse Ave., New Haven, Connecticut 06520-8283

E-mail address: anna.mazzucato@yale.com 